Historic, Archive Document

Do not assume content reflects current scientific knowledge, policies, or practices. 

to some

\section{Common Arizona}

Range Grasses
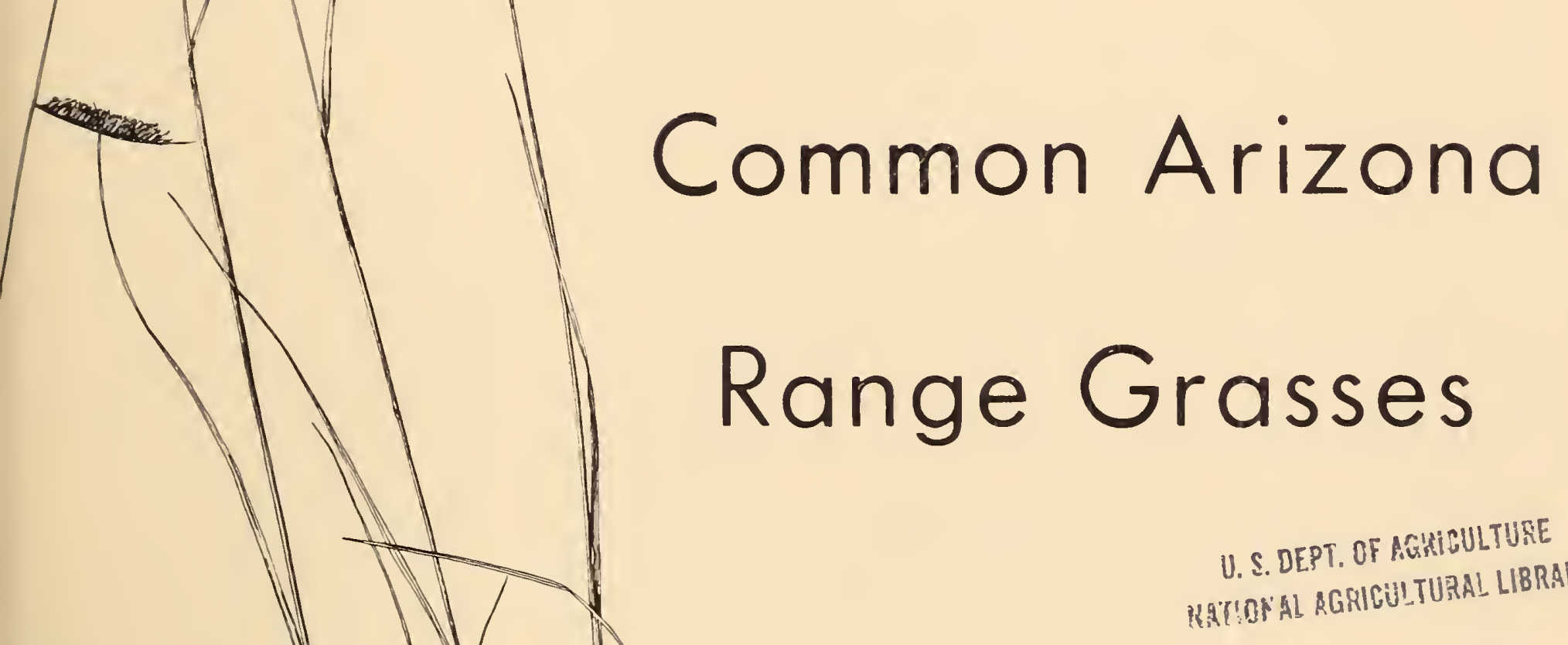
KATIOF AL RGRICULTURAL LIBRAR'

\section{AUG 211957}

CUREENT SERIAL RECOROS

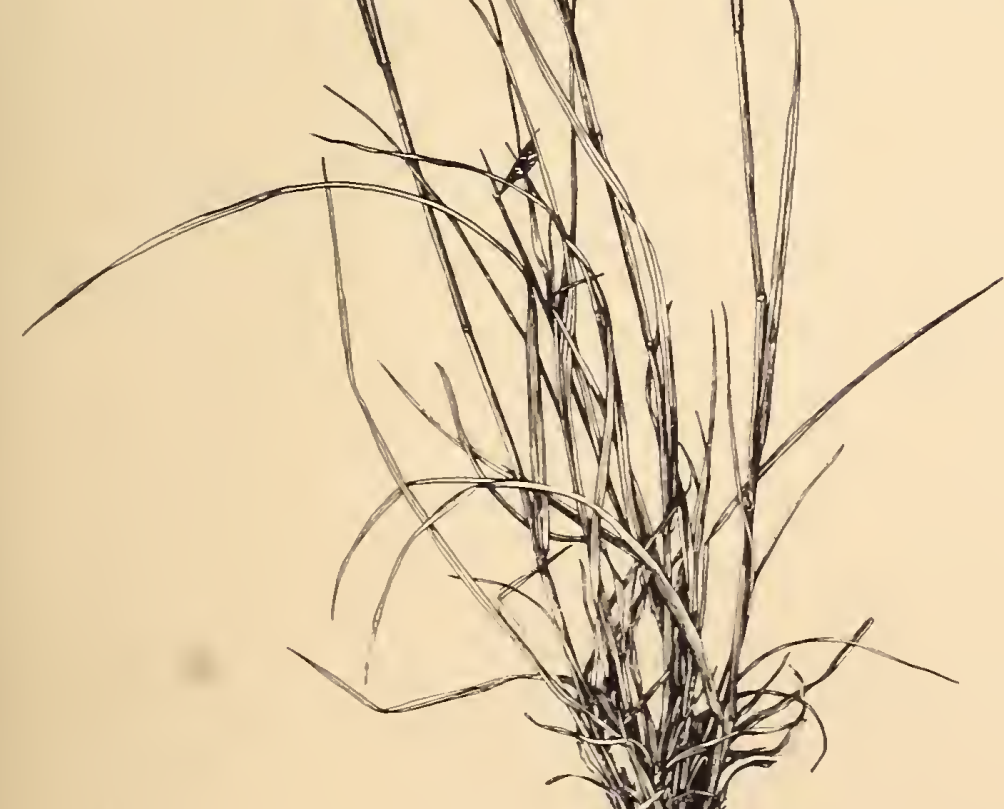

R. F. Copple and C. P. Pase

Rocky Mountain Forest and Range Experiment Station

Forest Service U. S. Department of Agriculture 
ABOUT THE COVER:

Hairy grama (Bouteloua hirsuta), a desirable forage grass characteristic of many southwestern ranges. 
U. S. Forest Service Research Paper RM-27

\section{A VEGETATIVE KEY}

TO

SOME COMMON ARIZONA RANGE GRASSES

by

R. F. Copple ${ }^{1}$

and

C. P. Pase, Plant Ecologist

Rocky Mountain Forest and Range Experiment Station ${ }^{2}$

1 Retired; formerly Range Conservationist, U. S. Bureau of Land Management, Reno, Nevada.

2 Central headquarters maintained in cooperation with Colorado State University at Fort Collins; Mr. Pase is located at Tempe in cooperation with Arizona State University. 


\section{CONTENTS}

\section{Page}

Introduction

Descriptions of vegetative characters .

How to use the key

Condensed key

Grasses grouped according to outstanding characters .

Vegetative key

Section A -- Plants, annual

Section B -- Plants, perennial • • • • • 13

Descriptions of species . • • • • • • • 26

Glossary • • • • • • • • • • • • • 65

Reference publications . • • • • • • • 69

Index to common and scientific names . . . . 70 


\title{
A Vegetative Key to Some Common Arizona Range Grasses
}

\author{
R. F. Copple and C. P. Pase
}

\section{INTRODUCTION}

Native and cultivated grasses must often be identified when plants are immature or have been heavily grazed and, thus, do not have flower stalks. Under such conditions, conventional botanical manuals offer little assistance. Examination of vegetative characteristics, however, will often permit tentative identification until better developed specimems are available.

The purpose of this paper is to present an illustrated key for the identification of 77 common Arizona range and pasture grasses by vegetative characters.

A technical knowledge of taxonomic botany, although useful, is not essential to use this method of identification. A common magnifying glass or hand lens of 5 to 10 power, plus a small millimeter scale, will prove helpful.

This publication is planned to be used in conjunction with Arizona Range Grasses (Ariz. Agr. Exp. Sta. Bull. 298), ${ }^{3}$ or with Hitchcock's Manual of the Grasses of the United States (U.S. Dep. Agr. Misc. Pub. 200). Photographs or drawings of the plants covered here may be found in these two publications.

\section{DESCRIPTIONS OF VEGETATIVE CHARACTERS}

Annual or perennial.--Annual grasses generally have fine, poorly developed root systems, and no evidence of old, previous year's culm bases. Perennial grass root systems are better developed, and evidence of last year's leaves or flower stalks can usually be found.

${ }^{3}$ Publications used for reference in preparing this Vegetative Key are listed on p. 69.
Presence of creeping root stalks, rhizomes, stolons, etc., always indicates a perennial grass. Seedling perennial grasses may occasionally be confused with annuals. Some caution should be used with this character in short-lived perennials such as Bouteloua rothrockii (plate I).

Vernation.--This refers to the cross section of a grass blade in a developing shoot. Vernation may be best determined by cutting across the sheath just below the collar, and observing the shape of the enclosed cut blade with a strong hand lens. Care should be used to make the cross section with a razor blade or sharp knife, to avoid mashing the enclosed blade and destroying its original shape. Vernation may be folded, rolled (or curled), or clasped (plate II).

Folded vernation is generally associated with a flattened sheath, and rolled or clasped with a rounded sheath, but this does not always hold true.

Blades.--Width and length of blades vary somewhat, depending on growing conditions, but within limits offer an easy method to separate certain species. Shaded plants have longer and wider blades than those exposed to the sun.

Nerves may be raised on either surface-sometimes prominently, as on the upper surface of Koeleria cristata blades.

Blades may be folded, flattened, or rounded. Normally flat blades may tend to appear round as they dry, but examination of the base of the blade above the sheath will reveal its normal condition. Blade tips may be boat-shaped, as in some species of Poa.

Grass blades are two-ranked, while sedges are three-ranked (plate I). 


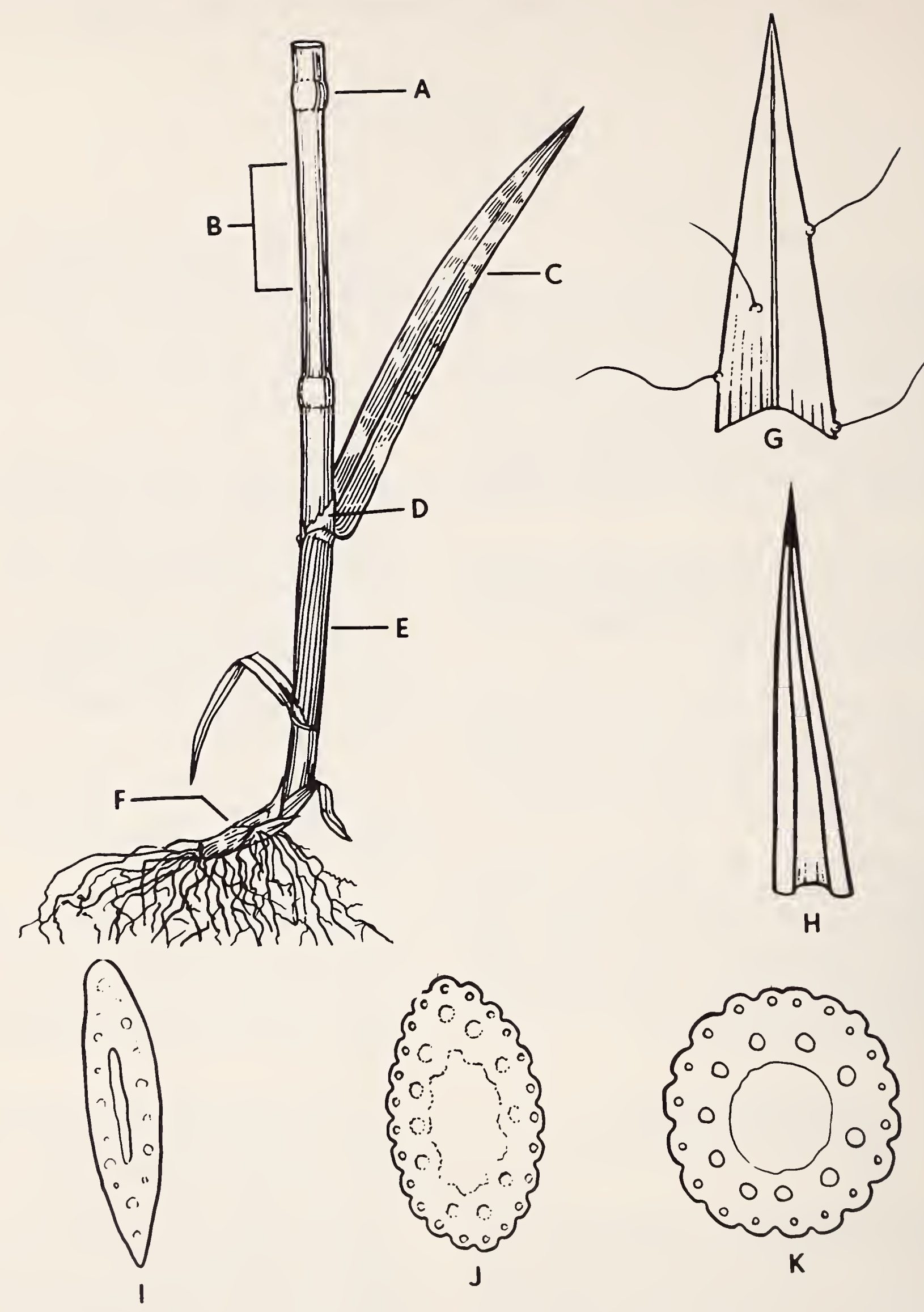

Plate I.--Grass identification characters.

Parts of typical grass plant: A, node; $B$, intemode; $C$, blade; $D$, ligule; $E$, sheath; $\underline{F}$, rhizome. Leaf blades: $G$, with glandular hairs; $\underline{H}$, with pungent tip. $\bar{C}$ ulm shape in cross section: I, flat; $\vec{J}$, elliptical; $\underline{K}$, round. 

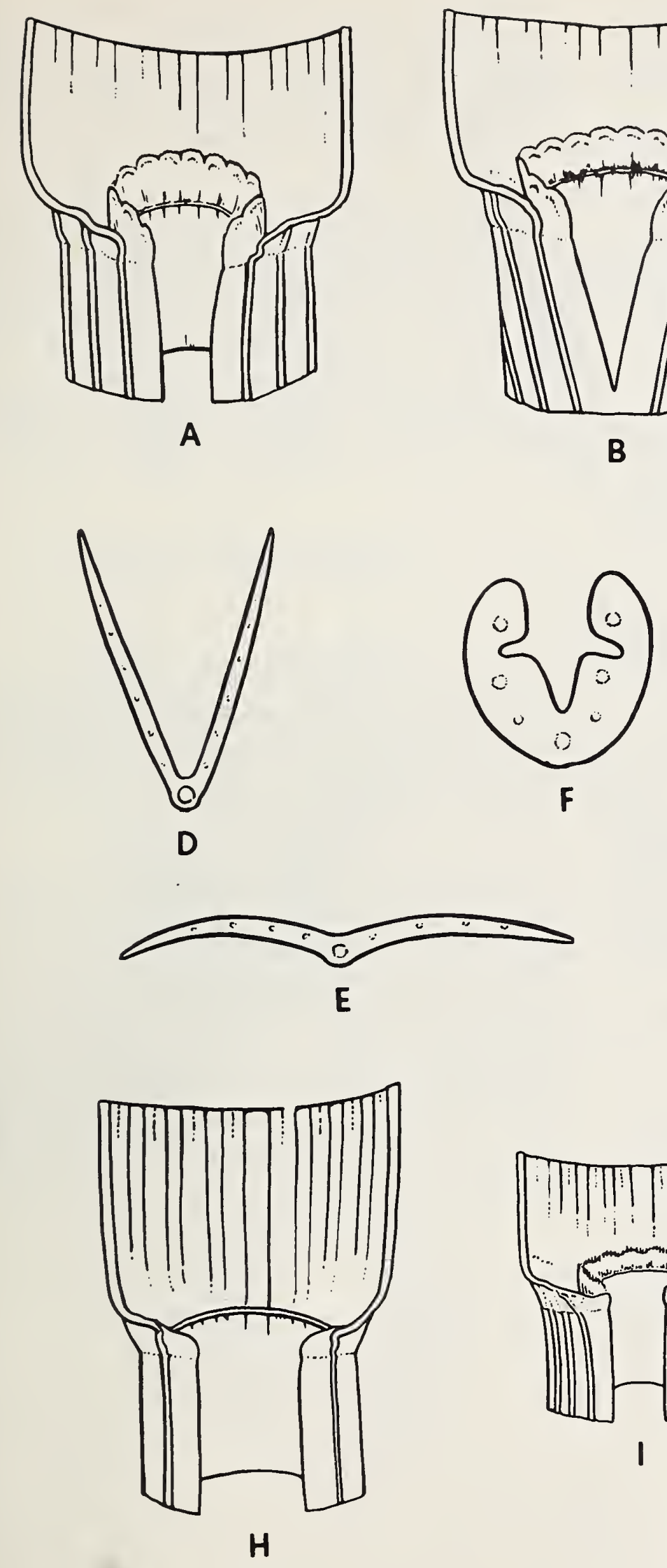

B
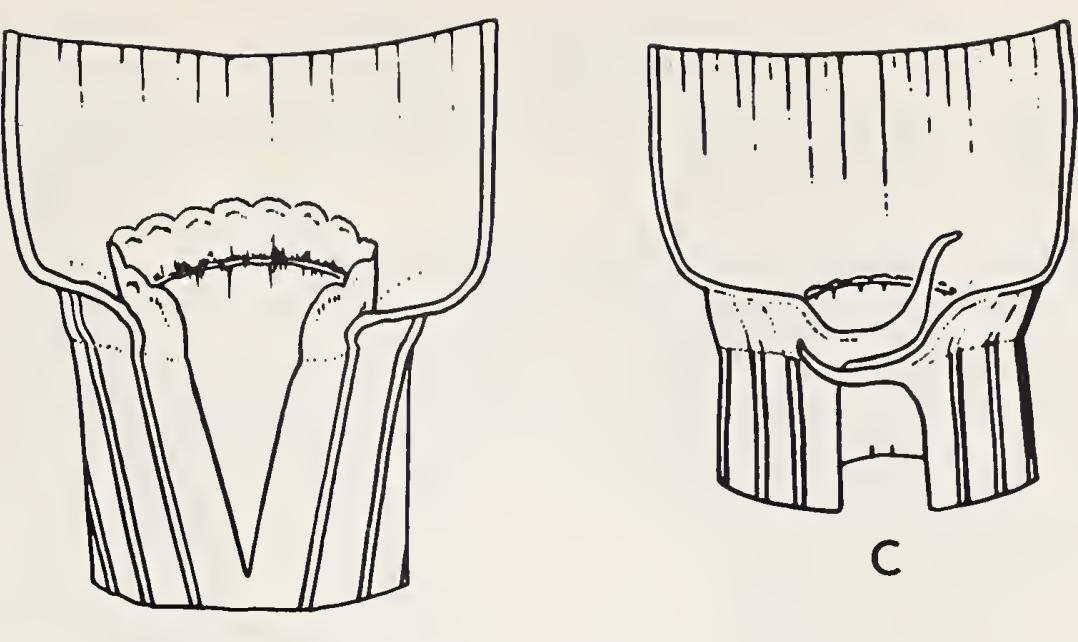

C

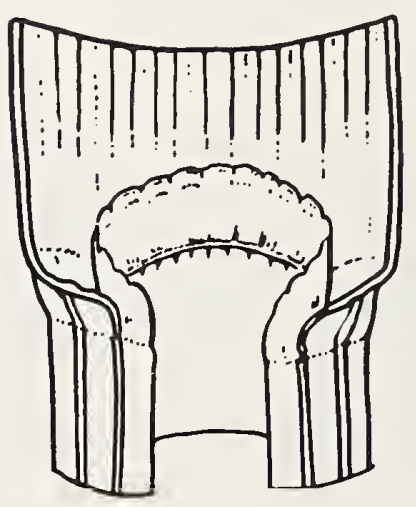

G

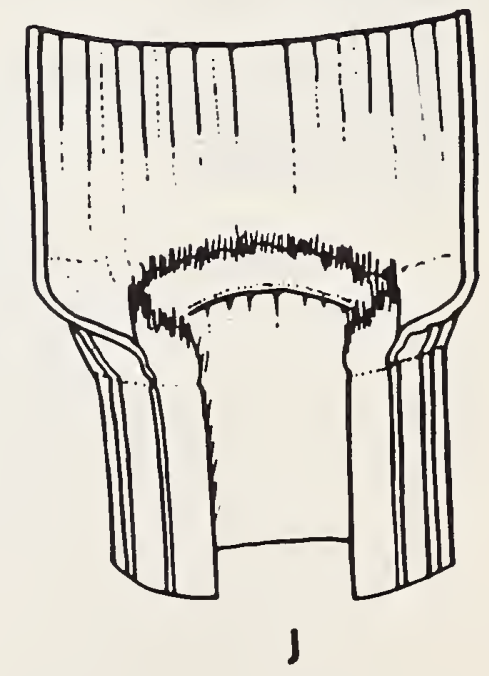

Plate II.--Grass identification characters.

Sheaths: A, open; $B$, closed; $C$, with clasping auricle. Leaf shape in cross section: D, folded; $\vec{E}$, flat; $\vec{F}$, round. $\overrightarrow{L i g u l e}$ types: $G$, membranous; $\underline{H}$, collar-like; I, hairy; J. tmuncate-ciliate (membranous with ciliate margin). 
Auricle.--Small, membranous extensions of the collar of some grasses, notably of Agropyron, Lolium, and others of the tribe Hordeae (plate II).

Ligule.--This is an organ clasping the stem at the junction of the blade and sheath. It may be membranous or hairy, or both, as in truncate-ciliate ligules. Size, shape, and type of ligule is quite constant, and provides one of the best vegetative characters for identification (plate III).

Collar.--This is the junction of leaf blade and sheath. It may be a broad or narrow band, glabrous or covered with fine hair. The margin may be glabrous, hairy, or with glandular hairs.

Sheath.--The tubular basal portion of the leaf, below the blade that clasps the stem. The sheath may be closed, as in Bromus, or open. Ruptured closed sheaths should not be confused with normally open ones (plate II).

The sheath of certain grasses may have a thin, membranaceous, hyaline margin.

Nodes.--The joints of a grass stem or culm. nodes.

Roots.--Grass root systems are fibrous, but rhizomes or thickened underground stems may also be present.
Culm.--The flowering stem of a grass, generally round or elliptical in cross section (plate I) as opposed to the triangular stems of sedges. Rushes have pithy stems, while most grasses have hollow stems.

Considerable variation may be expected in certain characters such as degree of hairiness of the blade or sheath, length and width of blade, etc. It may therefore be necessary to examine several specimens before deciding what is a representative condition. Other characteristics, such as type of ligule and vernation, are generally rather constant.

\section{HOW TO USE THE KEY}

To use the key, first examine the plant to see if it is annual or perennial. If perennial, go to Section B. Identification is then made by a series of opposing choices, until a name is reached in the key.

In the short-cut method, refer first to the condensed key which lists major characters with their number location in the key. For example, should the plant have stolons, go immediately to Item 10 in the key, and continue from there. Should this fail to bring you to an acceptable decision, start over at the beginning of the key. Remember, only 77 of the more common range grasses are covered by this key. 


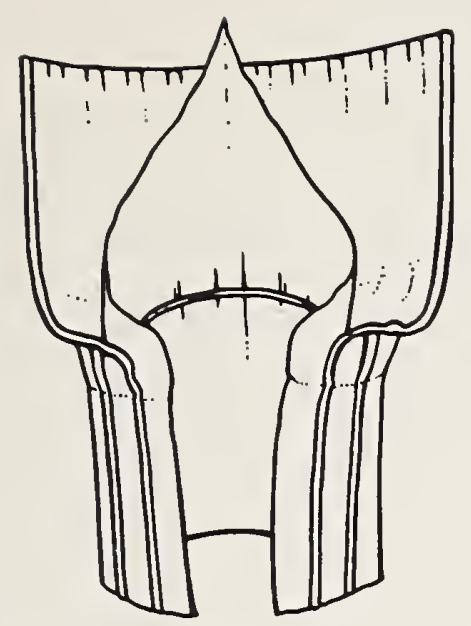

A
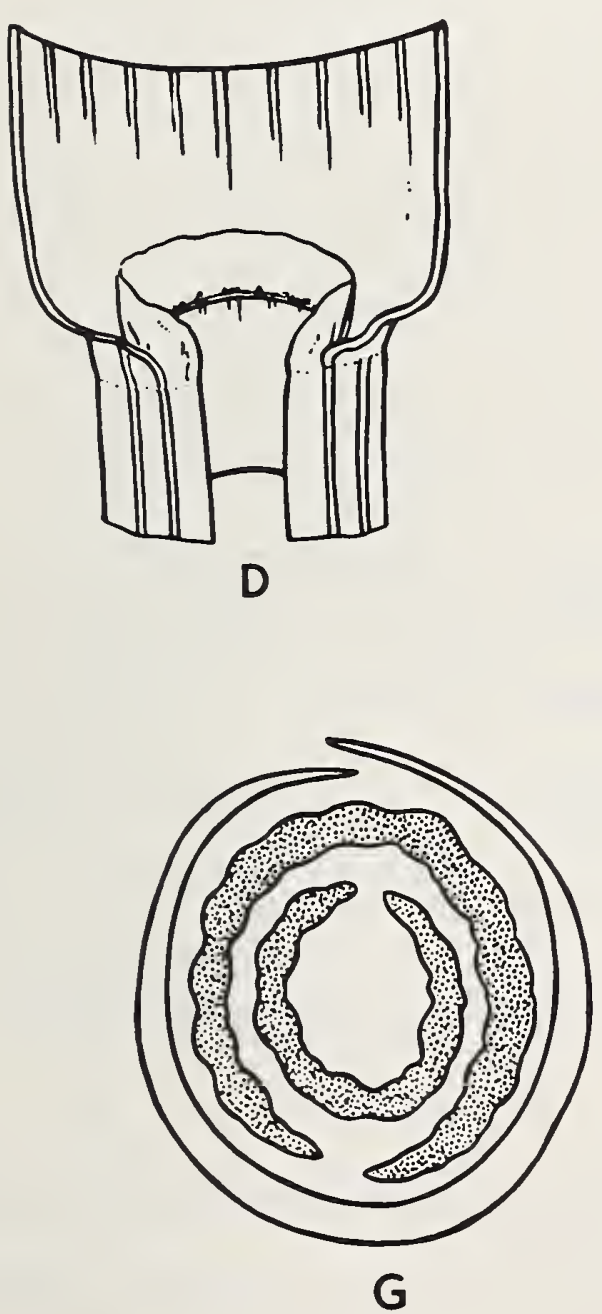

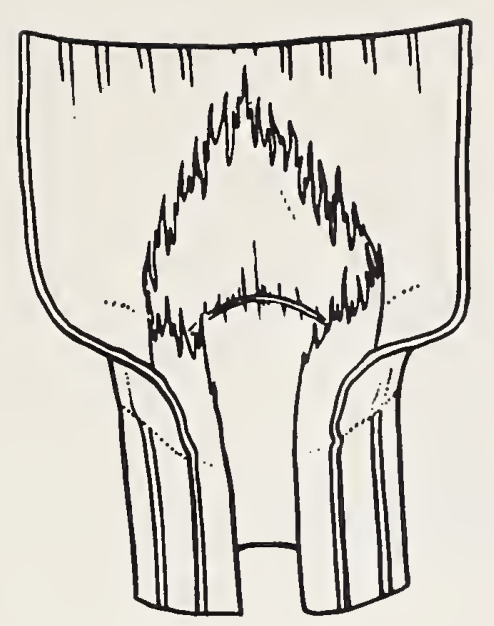

B
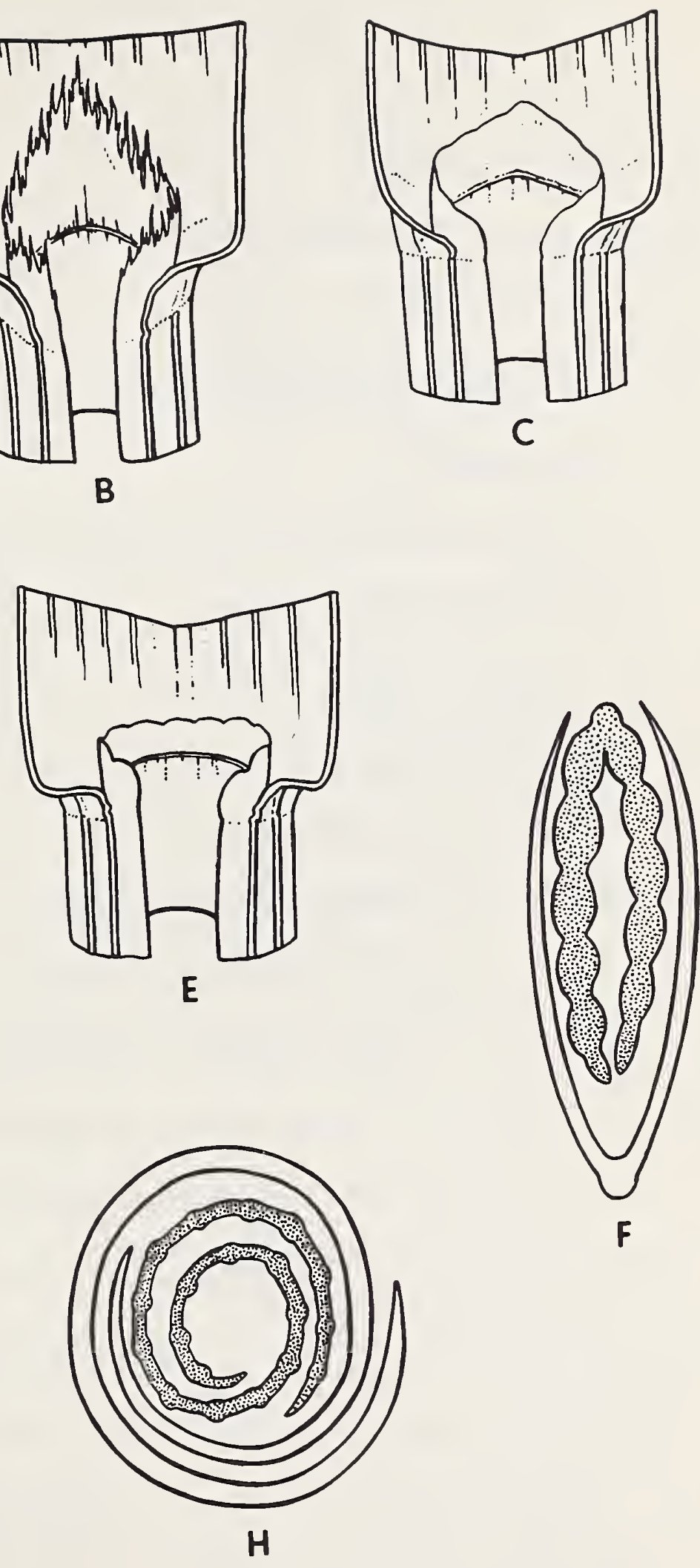

Plate III.-Grass identification characters.

Membranous ligule shapes: A, acute; B, acute-lacerate; $\underline{C}$, obtuse; D, truncate; E, truncate-notched. Vemation (cross section of leaf in bud): E, folded; $\underline{G}$, clasped; $\underline{H}$, curled or rolled. 


\section{Major Character Headings by Number in Key}

Section A - Annual

Section B - Perennial

1- Auricle present

3- Blade glossy on lower surface

5- Bulb present

6- Flowers and blades clustered at apex

7- Blade tip very sharp

8- Ligule large, 5-18 mm.

10- Stolons present

14,77-Rhizomes present

23- Sheath margin white

23,25- Blade margin white

28- Letter $\mathrm{M}$ or $\mathrm{W}$ on blade

29- Rachis extends beyond florets

30- Culms root at nodes

33,80 - Internode hairy or woolly

37- Blade margin with glandular hairs

42- Growth a mat or sod

44- Sheath closed partially or completely

46- Node halry, pubescent, or woolly

74- Panicle included in sheath 


\section{GRASSES GROUPED ACCORDING TO OUTSTANDING CHARACTERS}

1. Auricles

$$
\begin{aligned}
& 1 \text { - Agropyron desertorum } \\
& 2 \text { - Agropyron smithii } \\
& 66 \text { - Sitanion hystrix } \\
& 44 \text { - Lolium perenne }
\end{aligned}
$$

2. Stolons

$$
\begin{aligned}
& 31 \text { - Cynodon dactyzon } \\
& 32 \text { - Distichlis stricta } \\
& 38 \text { - Hilaria belangeri } \\
& 57 \text { - Panicum obtusum }
\end{aligned}
$$

3. Nodes Hairy or Pubescent

$$
\begin{aligned}
& 4 \text { - Andropogon barbinodis } \\
& 38 \text { - Hilaria belangeri } \\
& 41 \text { - Hilaria rigida } \\
& 50 \text { - Muhlenbergia pungens } \\
& 64 \text { - Setaria geniculata } \\
& 75 \text { - Trichachne californica } \\
& 76 \text { - Tridens muticus }
\end{aligned}
$$

\section{Blade Tip Very Sharp}

50 - Muhlenbergia pungens

5. Flowers Clustered at Apex

$$
77 \text { - Tridens pulchellus }
$$

6. Corm or Bulb

$$
55 \text { - Panicum bulbosum }
$$

7. Sheath Margin White

$$
\begin{aligned}
& 45 \text { - Lycurus phleoides } \\
& 51 \text { - Muhlenbergia richardsonis } \\
& 62 \text { - Schedonnardus paniculatus }
\end{aligned}
$$

8. Stems or Culm Root at Nodes

$$
19 \text { - Bouteloua eriopoda }
$$

$$
\begin{aligned}
& 38 \text { - Hilaria belangeri } \\
& 77 \text { - Tridens pulchellus }
\end{aligned}
$$

9. Rach1s Extends Beyond Spikelets

$$
22 \text { - Bouteloua hirsuta }
$$

10. Sheath Closed Partially or Completely

$$
\begin{aligned}
& 24 \text { - Bromus anomalus } \\
& 25 \text { - Bromus arizonicus } \\
& 26 \text { - Bromus ciliatus } \\
& 27 \text { - Bromus inermis } \\
& 28 \text { - Bromus mubens } \\
& 29 \text { - Bromus tectomm }
\end{aligned}
$$

11. Flowers Inserted in Sheath

$$
\begin{aligned}
& 69 \text { - Sporobolus asper } \\
& 70 \text { - Sporobolus cryptandrus }
\end{aligned}
$$

12. Internode Ha1ry

$$
\begin{aligned}
& 19 \text { - Bouteloua eriopoda } \\
& 33 \text { - Elyonums barbiculmis } \\
& 41 \text { - Hilaria rigida } \\
& 64 \text { - Setaria geniculata }
\end{aligned}
$$

13. Blade Margin with Glandular Hairs

$$
\begin{aligned}
& 15 \text { - Bouteloua aristidoides } \\
& 17 \text { - Bouteloua chondrosioides } \\
& 18 \text { - Bouteloua curtipendula } \\
& 19 \text { - Bouteloua eriopoda } \\
& 20 \text { - Bouteloua filiformis } \\
& 22 \text { - Bouteloua hirsuta } \\
& 23 \text { - Bouteloua rothrockii } \\
& 37 \text { - Heteropogon contortus } \\
& 38 \text { - Hilaria belangeri } \\
& 56 \text { - Panicum bulbosum }
\end{aligned}
$$


14. Growth a Mat or Sod-Like

$$
\begin{aligned}
& 2 \text { - Agropyron smithii } \\
& 21 \text { - Bouteloua gracizis } \\
& 31 \text { - Cynodon dactyzon } \\
& 38 \text { - Hizaria belangeri } \\
& 51 \text { - Muhzenbergia richardsonis } \\
& 53 \text { - Muhzenbergia torreyi } \\
& 56 \text { - Panicum bulbosum }
\end{aligned}
$$

15. Blade Margin White

$$
\begin{aligned}
& 4 \text { - Andropogon barbinodis } \\
& 45 \text { - Lycurus phleoides } \\
& 67 \text { - Sorghum halepense } \\
& 75 \text { - Trichachne californica }
\end{aligned}
$$

16. Blade Folded

$$
\begin{aligned}
& 5 \text { - Andropogon cirratus } \\
& 6 \text { - Andropogon scoparius } \\
& 44 \text { - Lolium perenne } \\
& 45 \text { - Lycurus phleoides } \\
& 47 \text { - Muhlenbergia emersleyi } \\
& 53 \text { - Muhlenbergia torreyi } \\
& 55 \text { - Panicum bulbosum } \\
& 59 \text { - Poa fendleriana } \\
& 60 \text { - Poa Zongizigula } \\
& 61 \text { - Poa pratensis } \\
& 62 \text { - Schedonnardus paniculatus } \\
& 64 \text { - Setaria geniculata }
\end{aligned}
$$

17. Rhizomes

$$
\begin{aligned}
2 & \text { - Agropyron smithii } \\
3 & \text { - Agrostis alba } \\
27 & \text { - Bromus inermis } \\
31 & \text { - Cynodon dactylon } \\
32 & \text { - Distichlis stricta }
\end{aligned}
$$

39 - Hilaria jamesii

40 - Hilaria mutica

51 - Muhlenbergia richardsonis

61 - Poa pratensis

67 - Sorghum halepense

18. Blade Glossy Ventrally

44 - Lolium perenne

19. Sheath Base Color Usually Pink

44 - Lozizm perenne

20. Blade A Bristle, Needle-Like, Round

8 - Aristida divaricata

9 - Aristida glabrata

10 - Aristida glauca

11 - Aristida Zongiseta

12 - Aristida purpurea

13 - Aristida ternipes

14 - Blepharoneuron tricholepis

33 - Elyonums barbiculmis

36 - Festuca arizonica

46 - Muhlenbergia curtifolia

48 - Muhlenbergia montana

50 - Muhlenbergia pungens

51 - Muhlenbergia pichardsonis

52 - Muhlenbergia migens

54 - Oryzopsis hymenoides

63 - Schismus barbatus

68 - Sporobolus airoides

73 - Stipa neomexicana

76 - Tridens muticus

77 - Tridens pulchellus

21. Letter $M$ or $W$ on Blade

27 - Bromus inermis 
22. Usually Glandular Hairs on Sheath

55 - Panicum bulbosum

56 - Panicum obtusum

23. Seeds Three-Awned

A - One long and two short

13 - Aristida temipes

B - Three of equal length

7 - Aristida adscensionis

8 - Aristida divaricata

9 - Aristida glabrata

10 - Aristida glauca

11 - Aristida Zongiseta

12 - Aristida purpurea

24. Single Awn

A - Short - one Inch or less

4 - Andropogon barbinodis

29 - Bromus tectorwm

30 - Chloris virgata

47 - Muhlenbergia emersleyi

48 - Muhlenbergia montana

49 - Muhlenbergia porteri

50 - Muhlenbergia pungens

67 - Sorghum halepense

B - Long awn - more than one Inch
a - Straight
66 - Sitanion hystrix
b - Twisted or genfculate
73 - Stipa neomexicana
74 - Stipa pringlei
37 - Heteropogon contortus

25. Stems Flat

4 - Andropogon barbinodis
6 - Andropogon scoparius

34 - Eragrostis intermedia

37 - Heteropogon contortus

43 - Leptochloa dubia

45 - Lycums phleoides

53 - Muhlenbergia torreyi

55 - Panicum bulbosum

60 - Poa longiligula

61 - Poa pratensis

62 - Schedonnardus paniculatus

65 - Setaria macrostachya

26. Culms Usually Branched

3 - Agrostis alba

4 - Andropogon barbinodis

7 - Aristida adscensionis

9 - Aristida glabrata

10 - Aristida glauca

13 - Aristida temipes

15 - Bouteloua aristidoides

18 - Bouteloua curtipendula

30 - ChZoris virgata

31 - Cynodon dactyzon

33 - Elyonums barbiculmis

35 - Eragrostis Zehmanniana

38 - Hilaria belangeri

41 - Hilaria rigida

45 - Lycums phleoides

49 - Muhlenbergia porteri

57 - Pappophorum mucronulatum

64 - Setaria geniculata

67 - Sorghwm halepense

69 - Sporobolus asper 
70 - Sporobolus cryptandrus

75 - Trichachne califormica

77 - Tridens pulchellus

27. Ligule Large - Acute

A - 2-5 mm.

25 - Bromus arizonicus
34 - Eragrostis intermedia

45 - Lycurus phleoides

59 - Poa Zongiligula

B - 5-8 mro.

48 - Muhlenbergia montana

C - 8-15 mm.

47 - Muhlenbergia emersleyi 


\section{VEGETATIVE KEY}

\section{Section A - Plants, Annual}

1a Ligule a membrane or collar (a truncate-ciliate ligule is classified as membranous).

2a Sheath closed.

3a Blades $4-6 \mathrm{~mm}$. wide ................25. Bromus arizonicus

3b Blades narrower, 1-4 mm. wide.

$4 a$ Ligule acute-lacerate; internodes sometimes puberulent near nodes; mature plants reddish brown. Lower chaparral and desert shrub formations below 6,000 feet ........28. Bromus mibens

4b Ligule obtuse-lacerate; internodes glabrous. Generally above 6,000 feet $. . \ldots \ldots \ldots \ldots \ldots \ldots . . .29$. Bromus tectorm

$2 \mathrm{~b}$ Sheath open.

5a Ligule truncate-c1liate, $1 \mathrm{~mm}$.; blades with dorsal glandular halrs; lower nodes geniculate; culms branched; awned. Below

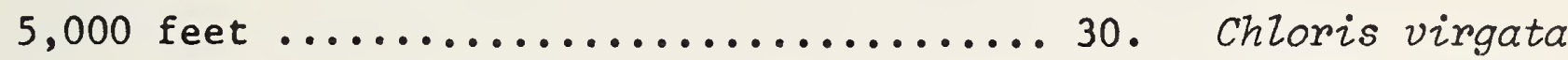

5b Ligule acute-1acerate, 2-4 mm. 1ong; blades 2-5 mm. wide 4-12 cm. 1ong. Desert to woodland 1,000-5,000 feet.

.................................... Poa bigelovii

lb Ligule hairy.

6 a Blades needle-1ike, short and decumbent, $1 / 2-1 \mathrm{~mm}$. wide, $3-8 \mathrm{~cm}$. long; long hairs back of 11gule and collar, 3-8 mm. 1ong. Dry slopes and sandy washes below 4,000 feet. 
6b Blade not needle-1ike.

7a Collar and blade with glandular hairs, 2-3 mm.; blade in bud folded, $1 / 2 \mathrm{~mm}$. wide, $1-2 \mathrm{~cm}$. long; ligule hairy, $1 / 2 \mathrm{~mm} . ;$ awns 1-2 cm. Below 6,000 feet. ......................... Aristida adscensionis

7b Collar without glandular hairs.

8a Blade margin with glandular hairs; culm elliptical; ligule hairy, 1/2 mm., blade in bud curled; stem branches; blades $1 / 2 \mathrm{~mm}$. wide, $2-7 \mathrm{~cm}$. long. Woodland below 6,000 feet. .......................... Bouteloua aristidoides

8b Blade margin without glandular hairs, flat, narrow and short, $1 \mathrm{~mm}$. wide, 2-4 cm. long; culm round, 1igule hairy, $1 \mathrm{~mm} . ;$ awns, 1-2 mm. Waste places throughout the State below 6,000 feet ...................... Bouteloua barbata 
la Auricle present, sometimes rudimentary.

2a Auricle prominent, clawed.

3a Blades glossy below; introduced plants of lawns, tame pastures ............................... Lolium perenne 3b Blades not glossy below.

4a Rhizomes present; blade ribs prominent dorsally; follage characteristically glaucous, blue-green.

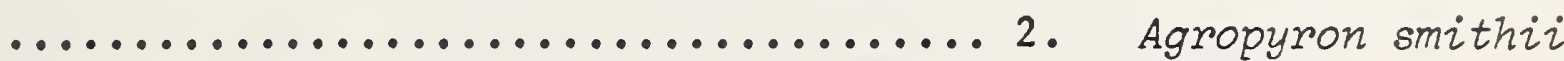

4b Rhizomes absent; blades conspicuously pubescent or rarely glabrous; tufted bunchgrass ........66. Sitanion hystrix

2b Auricle rudimentary ................ Agropyron desertomum 1b Auricle absent.

5a Culm-bases thickened into bulb-like corms.

\section{Panicum bulbosum}

5b Culm-bases not bulb-like.

6a Inflorescences and blades clustered at the apex, the blades exceeding the uppermost florets; plants small, blades involute, 2-5 cm. long, $1 / 4 \mathrm{~mm}$. wide, the plant 5-10 cm. high. ........................... 77. Tridens pulchelzus

$6 \mathrm{~b}$ Inflorescences and blades not clustered at apex.

7a Blade tip pungent, stiff and sharp; common on loose sand, woodland and pine areas ........50. Muhlenbergia pungens

$7 b$ Blade tips not sharp. 
8a Ligule large, 5-18 m.

9a Ligule acute-lacerate; culm elliptical; blades folded; large coarse bunchgrass ............47. Muhlenbergia emerszeyi

9b Ligule acute-entire; culm round; blades rolled, occasionally flat; small bunchgrass ..........48. Muhlenbergia montana

8b Ligule smaller, usually $1 / 2-3 \mathrm{~mm}$.

10a Stolons present.

11a Sheaths pubescent or hirsute.

12a Sheaths with glandular hairs...... 56. Panicum obtusum

12b Sheaths without glandular hairs... 31. Cynodon dactyion

11b Sheaths glabrous

13a Internodes woolly ............19. Bouteloua eriopoda

13b Internodes glabrous ............38. Hilaria belangeri

10b Stolons absent.

14a Rhizomes present.

15a Sheath closed, letter $M$ or $W$ on blade.

27. Bromus inermis

$15 b$ Sheath open.

16a Ligule of hairs.

17a Blades large, 10-12 m. wide, 20-40 cm. 1ong;

11gule 1-2 m.; rhizomes massive, often flattened;

fields, ditchbanks, roadsides.

67. Sorghum halepense

17b Blades smaller, 2-3 mm. wide, 1-6 cm. long,

frequently with salt deposits on upper surface.

32. Distichlis stricta 
16b Ligule membranous.

18. Blade folded, the tip boat-shaped; 11gule truncate-entire .............61. Poa pratensis

18b Blade not folded.

19a Plant woolly, the bases coarse, semiwoody; dry areas below 4,000 feet ...........41. Hilaria rigida

19b Plants not woolly.

20a Blades rolled, growth mat-like. ............. 51. Muhlenbergia richardsonis $20 \mathrm{~b}$ Blades not rolled, growth not mat-1ike.

21a Ligule truncate-ciliate, 1-2 mm.; blades 2-4 mm. wide, 5-10 cm. long, curled in the bud; culms branch at the nodes; dry, tight-soll flats, 2,000-6,000 feet, central and southern Arizona. ..................... Ho. Hizaria mutica

$21 b$ Iigule not truncate-ciliate.

22a Ligule truncate-lacerate, 1-2 m.; blade curled In the bud, involute, the base of blade usually flat, 2-5 m. wide, 4-12 cm. 1ong; dry flats on tight solls, central and northern Arizona, 4,500-7,000 feet....... 39. Hilaria jamesii

22b Ligule obtuse-entire, 1-2 m.; blades 2-5 m. wide, 3-8 cm. long; wet, cool sites generally along streams, 5,000-9,000 feet. 
$14 \mathrm{~b}$ Rhizomes absent.

23a Both sheath and blade margin white.

24 Blade folded, 11gule acute-entire, 2-5 man.; culms branched; small bunchgrass of dry woodland and pine, 4,000-7,000 feet ............45. Lycurus phleoides

$24 \mathrm{~b}$ Blade needle-11ke, 11gule truncate-toothed, growth mat-like ........... 51. Muhlenbergia richardsonis

23b Both sheath and blade margins not white.

25a Blade margins white.

26a Ligule hairy .............67. Sorghum haZepense

26b Ligule not hairy but membranous.

27 a Nodes woolly; 11gule a collar, obtuse-1acerate 2-3 m.; hairs on back of 11gule 3-5 mm.; medium to large bunchgrass; dry, rocky slopes $1,000-6,000$ feet ................4. Andropogon barbinodis

27b Nodes hairy but scarcely woolly, the culms erect from a knotty swollen felty-pubescent base, 40-100 cm. tall; sheaths glabrous to sparsely pilose; blades $3-5 \mathrm{~mm}$. wide, 8-12 cm. long .... 75. Trichachne califomica 25b Blade margins not white.

28a Blades with letters M or W.......27. Bromus inermis $28 \mathrm{~b}$ Blades not as above.

29a Rachis extends beyond florets.

22. Bouteloua hirsuta

29b Rachis does not extend beyond florets. 
30 a Culms root at nodes.

3la Blades with glandular hairs on upper side. 38. Hilaria belangeri

31b Blades without glandular hairs on upper side. 32a Internodes woolly ..... 19. Bouteloua eriopoda

32b Internodes not woolly, the ligule ciliate. ............... 77. Tridens pulchezlus $30 \mathrm{~b}$ Culms not rooting at nodes.

33a Internodes hairy or woolly, the ligules hairy. 34 Ligule $1 / 2 \mathrm{~mm} . . . . . .19 . \quad$ Bouteloua eriopoda $34 \mathrm{~b}$ Ligule larger, 1-2 mm.

35 a Blade rolled, $1 \mathrm{~mm}$. wide, $15-30 \mathrm{~cm}$. Iong. .............. 33. Elyonums barbiculmis

35b Blade not rolled, wider.

36a Blade flat, node woolly; 11gule obtuselacerate $2-3 \mathrm{~mm}$.

............. 4. Andropogon barbinodis

36b Blade folded; ligule, node and lower internode hairy.

64. Setaria geniculata

(see continuation) 
(continuation)

33b Internodes not halry nor woolly.

37a Blade margins, at least at the base, with glandular hairs. 38a Ligule membranous, 1-2 mm., collar with glandular hairs.

18. Bouteloua curtipendula

38b Ligule not membranous.

39a Culm flattened ............. 37. Heteropogon contortus

39b Culm round in corss section.

40a Ligule 2-4 m........... 23. Bouteloua rothrockii 40b Ligule smaller.

41a Blade in bud clasped ... 17. Bouteloua chondrosioides

41b Blade in bud curled ......20. Bouteloua filiformis

37b Blade margins without glandular hairs.

42a Growth a sod or mat-like.

43a Ligule hairy ............... 21 . Bouteloua gracilis

43b Ligule membranous ...........53. Muhlenbergia torreyi

$42 b$ Growth not a sod or mat-1ike.

44a Sheaths closed.

45a Ligule truncate-notched; culm round. ........................26. Bromus ciliatus

45b Ligule truncate-toothed, culm elliptical. ..........................24. Bromus anomalus

44b Sheaths open.

46a Nodes woolly or pubescent. 
47a Ligule obtuse-lacerate ..... 4. Andropogon barbinodis

$47 b$ Ligule not obtuse-lacerate.

48a Blades folded ............64. Setaria geniculata

48b Blades not folded.

49a Blades flat ..............41. Hilaria rigida 49b Blades round.

50a Blade small, about $1 \mathrm{~mm}$. wide, 2-5 cm. long;

Iigule truncate-ciliate; blade tip very sharp

to the touch; sandy soils of woodland and pine.

.................. 50. Muhlenbergia pungens

50b Blades larger, 2-4 mm. wide, 8-20 cm. long;

sheath margin hairy; blade in bud curled;

blade usually rolled; 1igule small, hairy;

desert grassland and oak woodland below

5,500 feet ............ 76. Tridens muticus

$46 \mathrm{~b}$ Nodes not woolly or pubescent.

51a Blades folded.

52a Growth a mat, ring-like....53. Muhlenbergia torreyi

52b Growth not mat-like or ring-like.

53a Ligule large, 8-15 mm. ..47. Muhlenbergia emersleyi

53b Ligule smaller.

$54 a$ Culm branched ..........45. Lycums phleoides

54b Culm not branched.

55a Ligule shorter than $1 \mathrm{~mm}$. 
56a Ligule obtuse-1acerate.

6. Andropogon scoparius

56b Ligule not obtuse-lacerate.

57a Ligule obtuse-toothed, about $1 / 2 \mathrm{~mm} . ;$

blades long, narrow, folded; woodlands

and pine forests, 5,000-11,500 feet,

rarely down to 3,500 feet.

59. Poa fendleriana

57b Ligule truncate-entire, 1/4-1/2 mo; bunch or sodgrass; common in molst meadows at medium and high elevations throughout the State ...............61. Poa pratensis

55b Ligule longer than $1 \mathrm{~mm}$.

58a Ligule obtuse-entire, 1-2 mm.; old growth usually flat, new growth blades folded, long, narrow, plant commonly has a reddish cast; woodland and pine forest fringe, 2,000-7,500 feet .............. 5. Andropogon cirratus

58b Ligule not obtuse-entire.

59a L1gule acute-1acerate, 2-4 mm.; blade $1 \mathrm{~mm}$. wide, 10-20 cm. long, common in chaparral, 3,500-7,500 feet .....6. 60. Poa Zongiligula

59b Ligule acute-entire, 2-3 mm.; blade margin white; woodland and pine, of ten on disturbed sites. 
51b Blade not folded.

60 a Blades round, needle-1ike.

6la Ligule a collar or membranous.

62 Iigule large, 5-8 mm., acute-ent1re; blade in bud folded, a small bunchgrass, common in woodland and plne forests; 4,500-9,500 feet. ...................48. Muhzenbergia montana

62b Ligule smaller.

63a Ligule less than $1 \mathrm{~mm}$. 1ong.

$64 a$ Ligule truncate-c111ate.

65a Ligule with hairs on back, 1-2 mm.; blade in bud curled; short rhizomes often present; large coarse bunchgrass of alkall flats; 2,500-6,500 feet ....68. Sporobolus airoides

65b Ligule with no hairs on back, truncate-c1liate, 1/4-1/2 mm.; blade clasped in the bud; culms occasionally branched at nodes; small bunchgrass of dry upland sites; 3,500-6,000 feet. ....................10. Aristida glauca

$64 \mathrm{~b}$ Ligule not truncate-c111ate.

66 Ligule obtuse-c1liate, $1 / 4 \mathrm{~mm} . ;$ blade $1 / 2 \mathrm{~mm}$. wide, 25-35 cm. long, needle-11ke, awns long, twisted, with long hairs extending to the tip. 73. Stipa neomexicana 
67 Ligule truncate-entire, $1 / 4 \mathrm{~mm} . ;$ blade

$1 \mathrm{~mm}$. wide, 5-10 cm. long; small bunchgrass of pine forests, 6,000-10,000 feet, occasionally lower ....... 14. Blepharoneuron tricholepis

$67 \mathrm{~b}$ Iigule acute-entire, very smal1, 1/4-1/2 mm.; blade 1/2-1 m. wide, 10-30 cm. long; large bunchgrass of forest, parks and dry meadows, $7,000-10,000$ feet

36. Festuca arizonica

63b Ligule longer than $1 \mathrm{~mm}$.

68a Ligule lacerate.

69a Blade veins each side of midvein 2; blade in bud clasped; sheath margin whtte; large coarse bunchgrass, commonly on moist, rocky sites along intermittent streams; 3,000-7,000 feet. 52. MuhZenbergia rigens

69b Blade veins each side of midvein 3-4; juniper and pine, $6,500-8,000$ feet.

46. Muhlenbergia curtifolia

68b Ligule entire, 2-3 mm.; collar margin pubescent; blade $1 / 2 \mathrm{~mm}$. wide, 5-20 cm. long; common in juniper-pinyon, 3,500-6,500 feet. 
61b Ligule of hairs.

70a Blade in bud clasped.

71a Ligule and collar bearded; dry sites; woodland to pine, 4,000-7,000 feet.

\section{Aristida divaricata}

$71 \mathrm{~b}$ Ligule not bearded.

72a Awns 2-4 cm. long; culms branch freely; blade halrs on upper surface $2-3 \mathrm{~mm}$. ................9. Aristida glabrata

72b Awns 5-8 cm. 1ong; culms unbranched; blades glabrous on upper surface.

...............11. Aristida Zongiseta

$70 \mathrm{~b}$ Blade in bud folded.

73a Collar bearded; blades $1 / 2 \mathrm{~mm}$. wide, 2-8 cm. long; awns 2-4 mm., with purplish cast; blades rolled, needle-1ike, desert to pine, 1,000-7,000 feet ..............12. Aristida purpurea

73b Collar not bearded, awns 1 long, 2 short; culm unbranched; collar with hairy margin only; culms elliptical; dry uplands; 2,500-5,500 feet. .................... Aristida ternipes

$60 \mathrm{~b}$ Blades flat, not round or needle-like.

74 a Panicle exserted, not included in the sheath. 75a Ligule of hairs for half or more its length. 
$76 a$ Blades sparsely pilose to pubescent, at least below.

77a Plants with rhizomes, forming an open to dense sod; blades narrow.

78a Blades rolled; salt crystals often on blades; lower culms and rhizomes conspicuously shiny. .............. 32. Distichlis stricta

78b Blades flat, the older ones often curled; salt deposits wanting; culms and rhizomes not as above......21. Bouteloua gracizis

77b Plants without rhizomes; blades 4-5 mm. wide. .................43. Leptochzoa dubia

76b Blades glabrous.

79a Sheaths pubescent on the margin or summit.

$80 \mathrm{a}$ Nodes and internodes glabrous; collar margins distinctly tufted; culms round. ............. 71. Sporobolus giganteus

$80 \mathrm{~b}$ Nodes and internodes sparsely hairy; collar margin not tufted; culms flat. ............65. Setaria macrostachya $79 \mathrm{~b}$ Sheaths glabrous.

81 a Culms branched, blades 12-15 cm. Iong. ........... 35. Eragrostis Zehmanniana

81b Culms simple, blades 45-60 cm. 1ong. 72. Sporobolus wrightii 
75b Ligule membranous, sometimes ciliate.

$82 \mathrm{a}$ Leaf folded in the bud; veins prominent ventrally. Small bunchgrass ....... 42. Koeleria cristata

$82 \mathrm{~b}$ Leaf rolled in the bud.

83a Culms branched.

$84 \mathrm{a}$ Sheaths and nodes pubescent. Introduced grass ........ 35. Eragrostis Zehmanniana

$84 \mathrm{~b}$ Sheaths and nodes glabrous.

85a Wiry decumbent bunchgrass, often clambering over shrubs; blades $3-8 \mathrm{~cm}$. long. ........... 49. Muhzenbergia porteri

85b Erect bunchgrass, blades 15-30 cm. 1ong. ......... 57. Pappophomm mucronulatum

83b Culms not branched.

$86 a$ Nodes pubescent; 11gule obtuse-lacerate. ................. 74. Stipa pringlei

$86 \mathrm{~b}$ Nodes glabrous; 11gule truncate-cillate. ............. 34. Eragrostis intermedia

74b Panicle partly or wholly covered by the sheath.

87 a Culm branched ...........69. Sporobolus asper

$87 \mathrm{~b}$ Culms not branched .... 70. Sporobolus cryptandms 


\section{DESCRIPTIONS OF SPECIES}

1. Agropyron desertorum (Fisch.) Schult. CRESTED WHEATGRASS

Erect, medium size bunchgrass. VERNATION - Curled. BLADES - Flat, 2-5 mme wide, 8-20 cm. long; semi-erect; rough ventrally; veins each side of midrib usually three; ribs prominent dorsally, 12-18; blade margin toothed. AURICLE Small, often rudimentary. LIGULE - Membranous, collar-like, $1 / 2 \mathrm{~mm}$. long. COLLAR - Glabrous. SHEATH - Glabrous, papery margin. NODES - Glabrous. INTERNODES - Glabrous. ROOTS - Fibrous. CULM - Round, unbranched. OUTSTANDING CHARACTERS - Small auricle, growth in very early spring.

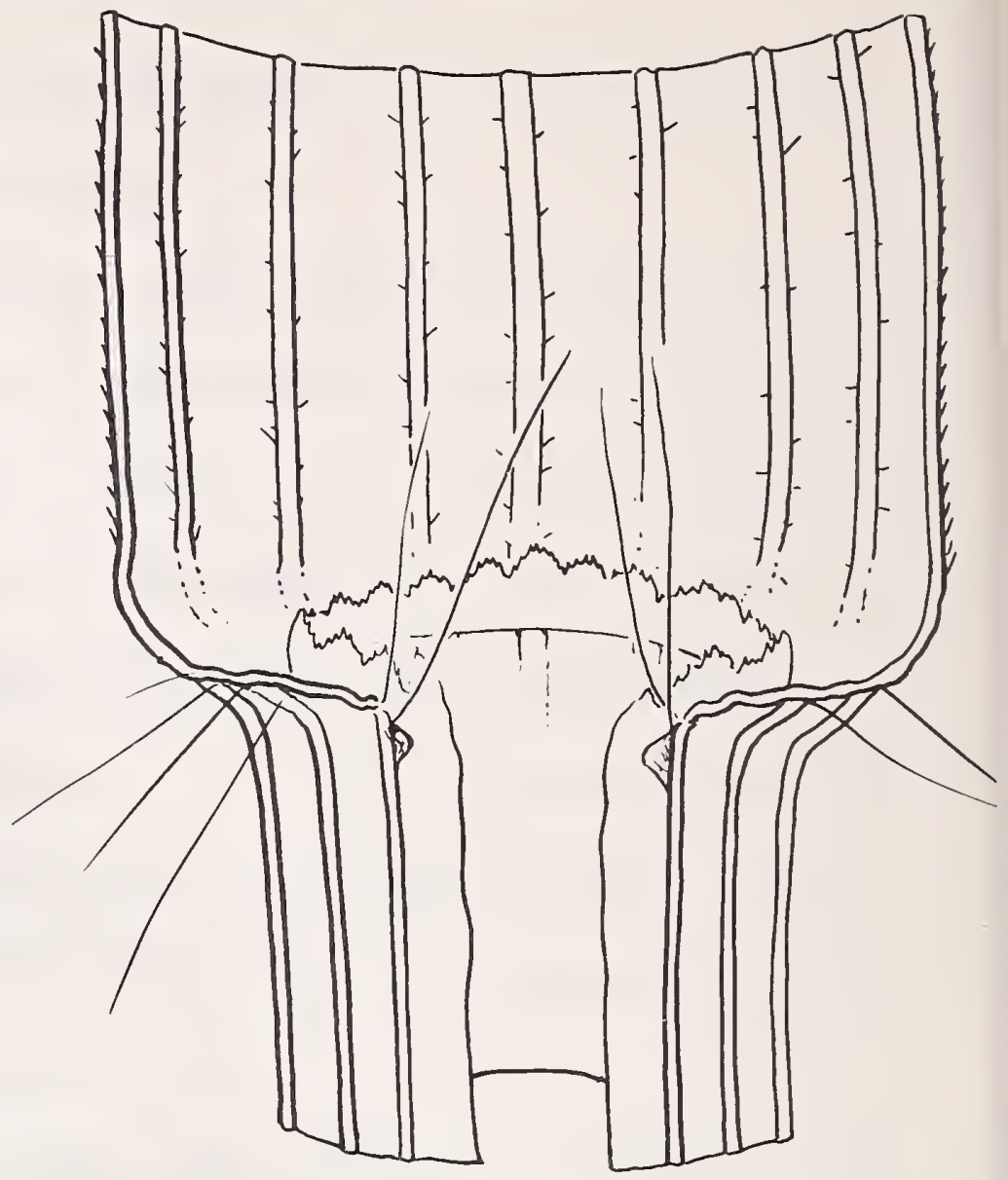

2. Agropyron smithii Rydb. WESTERN WHEATGRASS

Erect, individual plants to an open sodgrass. VERNATION - Curled. BLADES - Usually rolled blue-green, erect, narrow pointed; rough dorsally, pubescent ventrally; veins each side of midrib, 6-8; ribs prominent ventrally; margin toothed, midrib not prominent; $2-3 \mathrm{~mm}$. wide, $5-15$ cm. long. AURICLE - Small, 1/4-1/2 mm., usually brownish-red. LIGULE Collar-like (membranous), small, 1/4-1/2 mm.; truncate-toothed. COLLAR - Smooth, divided. SHEATH - Smooth, round, veined; lower pinkish to red. NODES - Glabrous. INTERNODES - Glabrous. ROOTS - Fibrous, rhizomatous. CULM - Round, not branched. OUTSTANDING CHARACTERS Auricles small, blade ribs prominent dorsally, growth usually sod, blade color usually a blue-green.

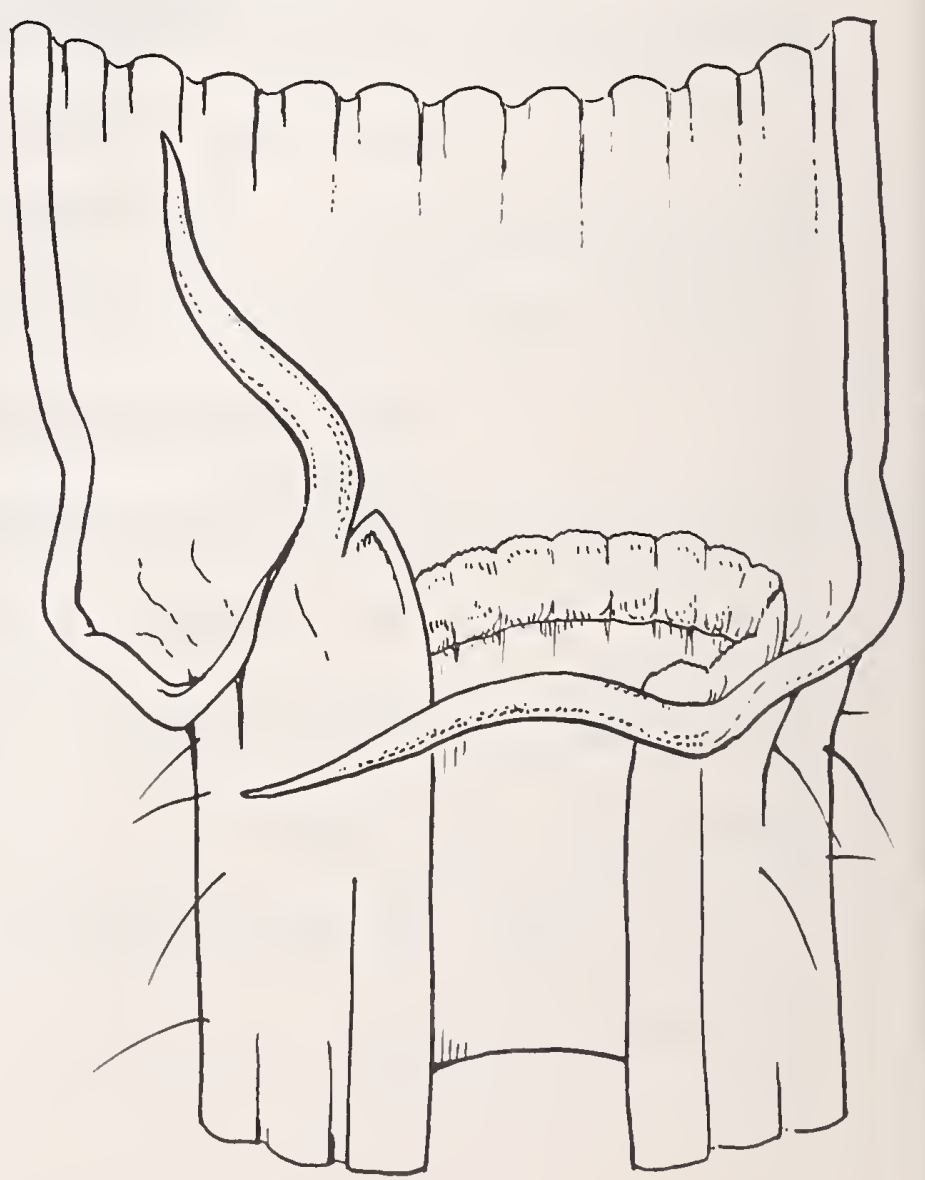


Erect, open sod. VERNATION - Folded erect, narrow, pointed, wider at base; rough ventral and dorsal; veins each side midrib, 3; ribs prominent dorsally and ventrally; margin toothed; midrib prominent dorsally; 3-5 mm. wide, $3-8 \mathrm{~cm}$. long. AURICLE - None. LIGULE - Menbranous, small, 1-3 mm., obtuse-entire to slightly toothed. COLLAR - Glabrous, divided. SHEATH - Glabrous, round, frequently purplish-red. NODES - Glabrous. INTERNODES - Glabrous. ROOTS - Rhizomatous. CULM - Round, occasionally branched. OUTSTANDING CHARACTERS Ligule small, 1-2 nm.; obtuse-entire, blades flat, wide tapered to point, glabrous, culm frequently reddish-purple at maturity, rhizomatous. (also reported as curled). BLADES - Flat,

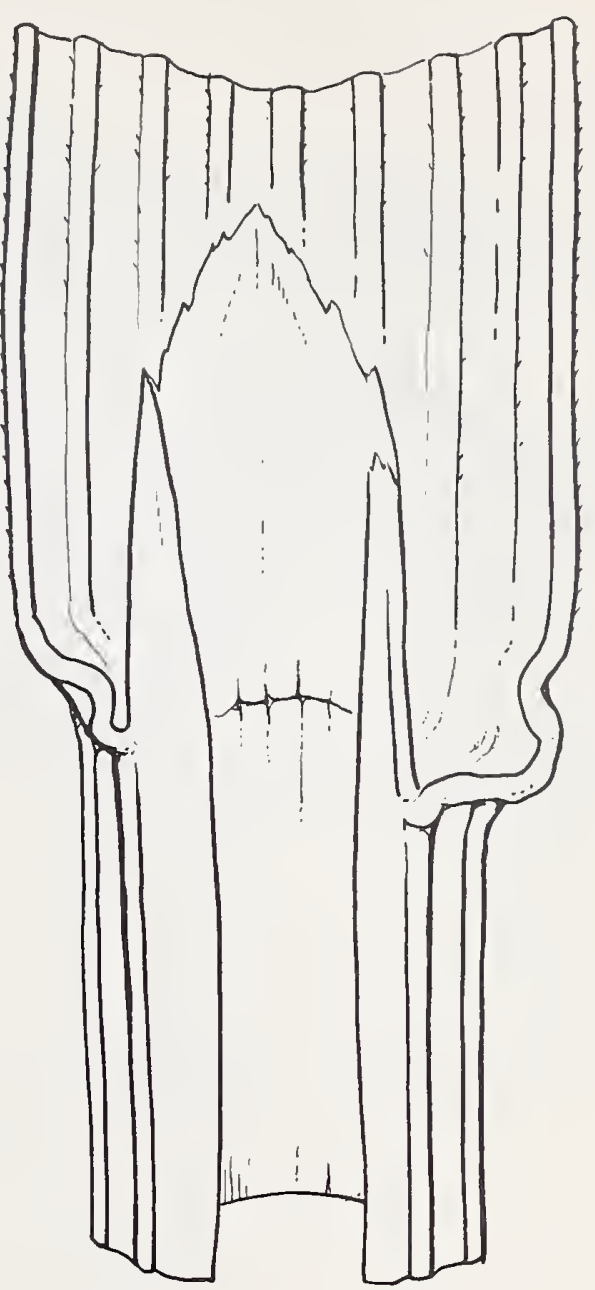

4. Andropogon barbinodis Lag. CANE BLUESTEM

Erect, small bunchgrass. VERNATION - Curled. BLADES - Flat, narrow, pointed; reddish; rough ventrally and dorsally; margin white, toothed; midrib prominent dorsally; 2-5 mm. wide, 8-20 cm. long. AURICLE - None. LIGULE - Membranous, 2-3 mm., obtuse-lacerate, occasionally long hairs on back, 3-5 mm. COLLAR Smooth, divided. SHEATH - Round, reddish, smooth, hyaline margin, veined. NODES - Woolly. INTERNODES - Glabrous. ROOTS - Fibrous. CULM - Round, usually branched, reddish. OUTSTANDING CHARACTERS - Blade margin white; nodes woolly, culms large, usually branched. Mature plants usually reddish. Panicle large, cotton-like, awned.

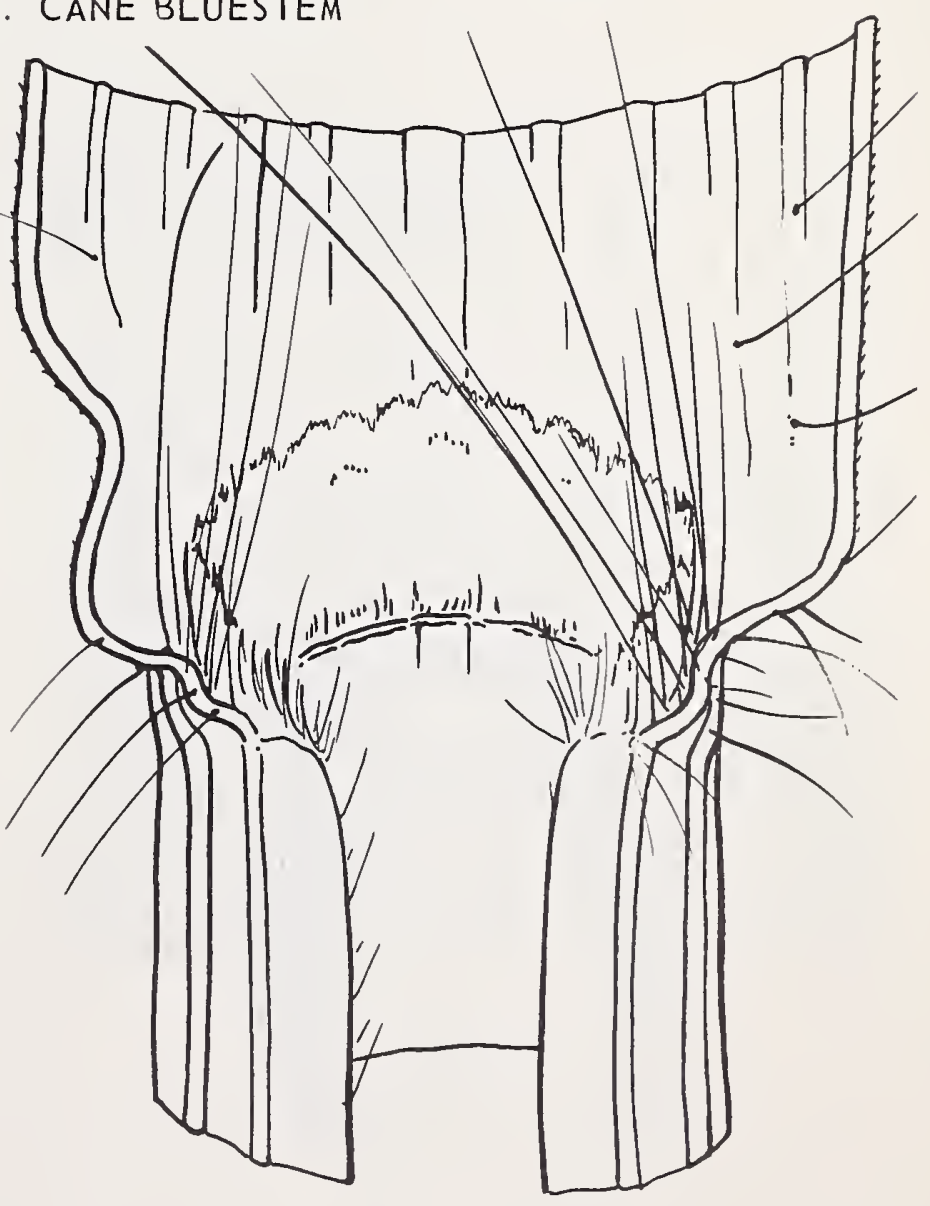


Erect, small bunchgrass。VERNATION - Folded. BLADES - Old, flat; new, folded; erect, narrow, pointed; veins each side of midrib 2-3; midrib prominent dorsally; 2-4 mm. wide, 12-20 cm. long. AURICLE None. LIGULE - Membranous, small, 1-2 mm., obtuse-entire. COLLAR - Glabrous. SHEATH - Glabrous, round. NODES - Glabrous。INTERNODES - Glabrous。ROOTS Fibrous. CULM - Round, sparingly branched. OUTSTANDING CHARACTERS Old growth blades usually flat. New growth blades folded, long, narrow.

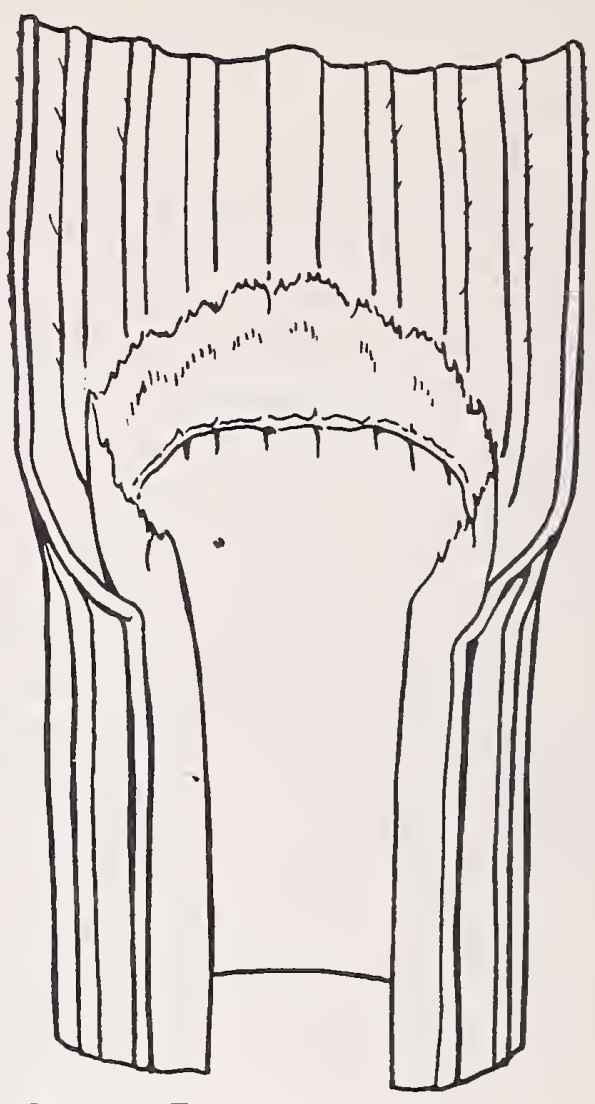

6. Andropogon scoparius Michx. LITTLE BLUESTEM

Erect, medium to large bunchgrass. VERNATION - Folded. BLADES - Flat to folded, narrow, dropping, pointed; rough, margin toothed; veins each side of midrib usually 4; ribs prominent ventrally and dorsally; midrib prominent dorsally; $2 \mathrm{~mm}$. wide, 5-20 cm. long. AURICLE - None. LIGULE - Collar-like (membranous), small, 1/2 mm., occasionally to $2 \mathrm{~mm}$., obtuse, finely lacerate. COLLAR - Glabrous, very small. SHEATH - Glabrous, flat, occasionally pinkish. NODES - Glabrous, reddish-brown at maturity. INTERNODES - Glabrous. ROOTS - Fibrous. CULM - Flat. OUTSTANDING CHARACTERS - Blades usually folded, long and narrow, twisted. Stems elliptical to flat. Ligule very small and difficult to observe.

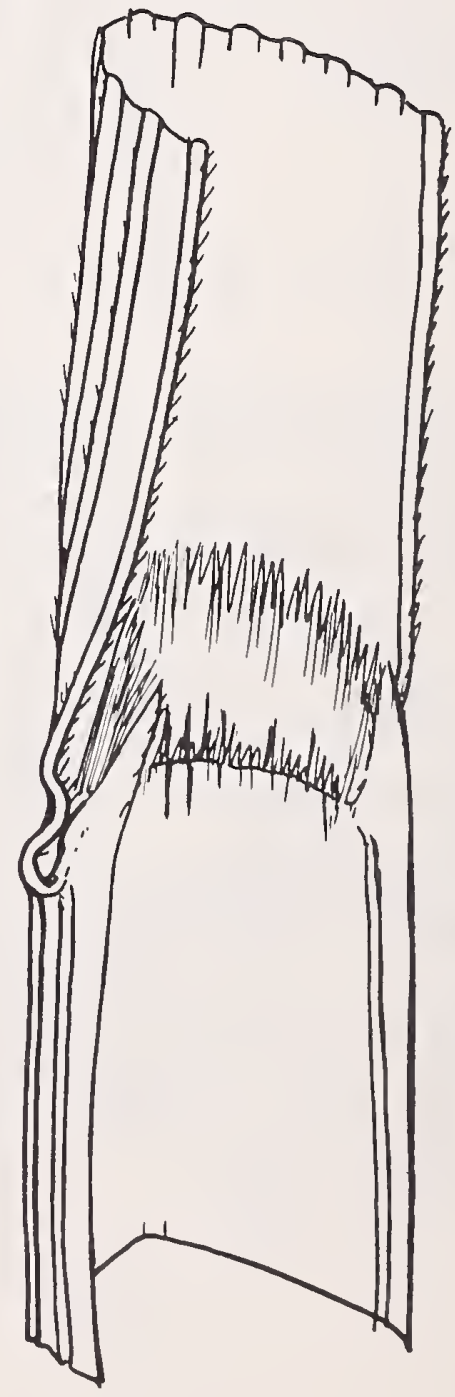


Annual, erect, small bunchgrass. VERNATION - Folded. BLADES - Flat, narrow, short; glabrous, soft; veins each side of midrib, 3-4; ribs not prominent; margin glandular hairs at base; nidrib not prominent; $1 / 2-1 \mathrm{~mm}$. wide, $1-21 / 2 \mathrm{~cm}$. long. AURICLE - None. LIGULE - Hairy, small $1 / 2 \mathrm{~mm}$., long hairs, $2.3 \mathrm{~mm}$. COLLAR Hairy margin, glandular, 2-3 mm. SHEATH - Occasional hairs, papery margin. NODES - Glabrous. INTERNODES - Glabrous. ROOTS - Fibrous, superficial. CULM Round, frequently branched. OUTSTANDING CHARACTERS - Annual. Collar with glandular hairs. Ligule hairy, very small $1 / 2 \mathrm{~mm}$. Roots superficial. Blades narrow, short, usually margin glandular hairs. At maturity usually blown away. Awns, 1-2 $\mathrm{cm}$.

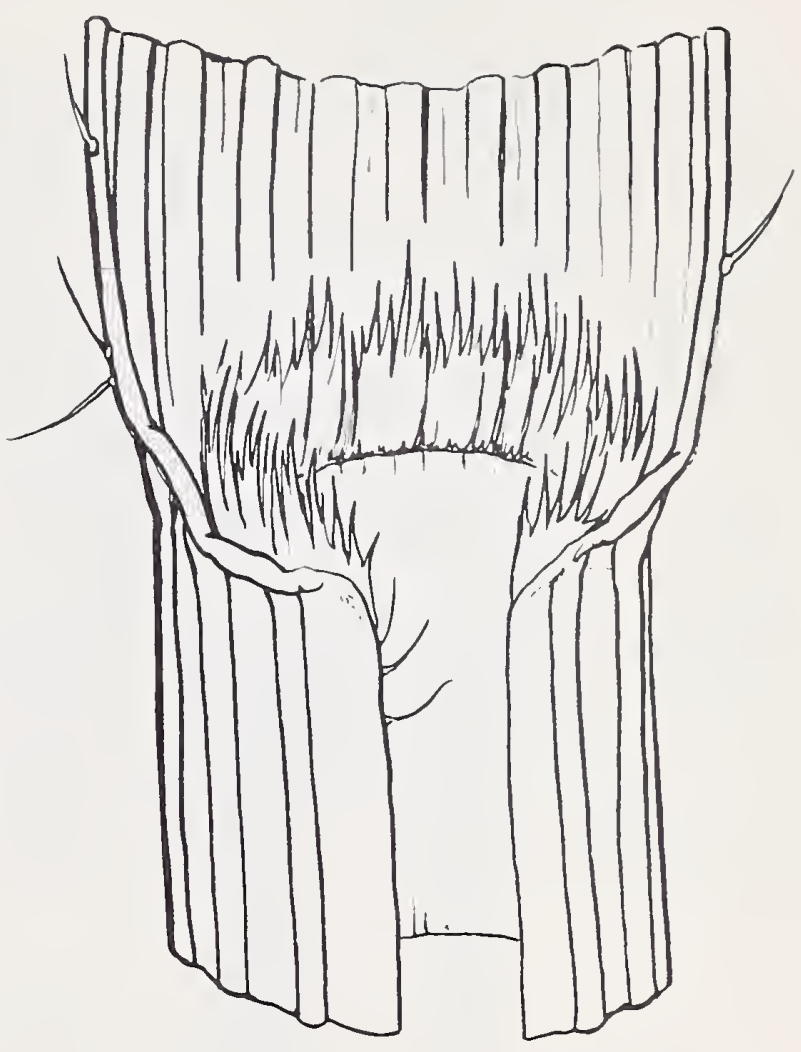

8. Aristida divaricata Humb. \& Bonpl. POVERTY THREE-AWN

Erect, small bunchgrass. VERNATION - Clasped. BLADES - Rolled, erect, narrow, pointed; smooth; ribs prominent ventrally; margin toothed; midrib not prominent; $1 / 2-1 \mathrm{~mm}$. wide, 5-15, occasionally $25 \mathrm{~cm}$. long. AURICLE - None. LIGULE Hairy, small 1/2-1 mm. COLLAR B earded, 1-2 mm. SHEATH - Glabrous, veined. NODES - Glabrous. INTERNODES - Glabrous. ROOTS - Fibrous. CULM Elliptical, not branched. OUTSTANDING CHARACTERS - Collar and ligule bearded. Glades narrow, rolled, thread-like. Awns short 10-15 $\mathrm{mm}$.

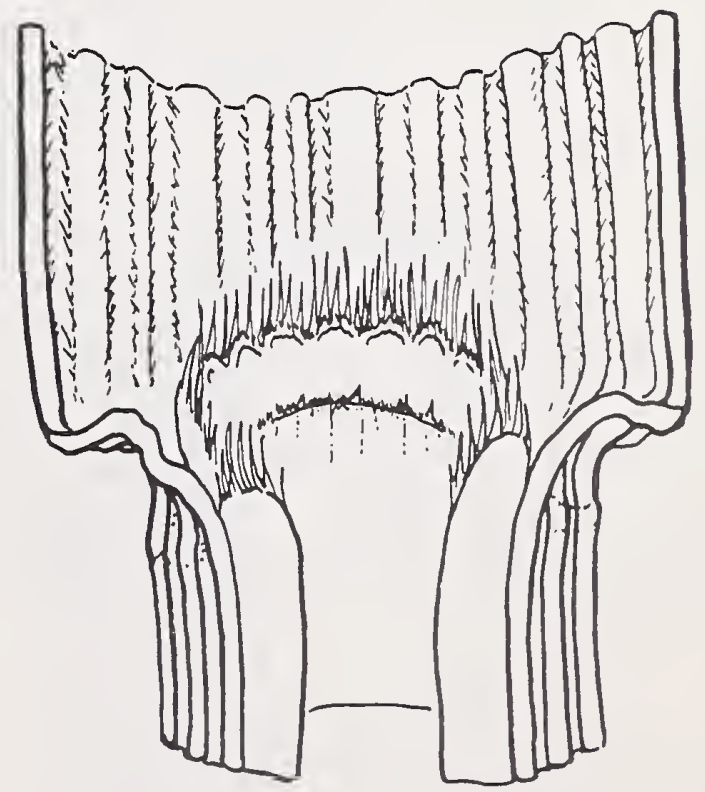


Erect, small bunchgrass. VERNATION - Clasped. BLADES - Hairy ventrally on lower $1 / 4,2-3 \mathrm{~mm}$; veins each side of midrib, 3; ribs prominent ventrally and dorsally; margin glabrous; midrib not prominent; $1 \mathrm{~mm}$. wide, $5-18 \mathrm{~cm}$. long. AURICLE - None. LIGULE - Hairy, small 1/2-1 mm., occasional hairs 2-3 $\mathrm{mm}$. COLLAR - Glabrous, divided. SHEATH Glabrous, veined, papery margin. NODES Glabrous. INTERNODES - Glabrous. ROOTS - Fibrous. CULM - Elliptical, strongly branched. OUTSTANDING CHARACTERS - Blades long, narrow, usually rolled. Ligule hairy, small, and scattered hairs, 2-3 mm. Blades scattered hairs, 2-3 mm. dorsally. Awns, 2-4 cm. Culms branched.

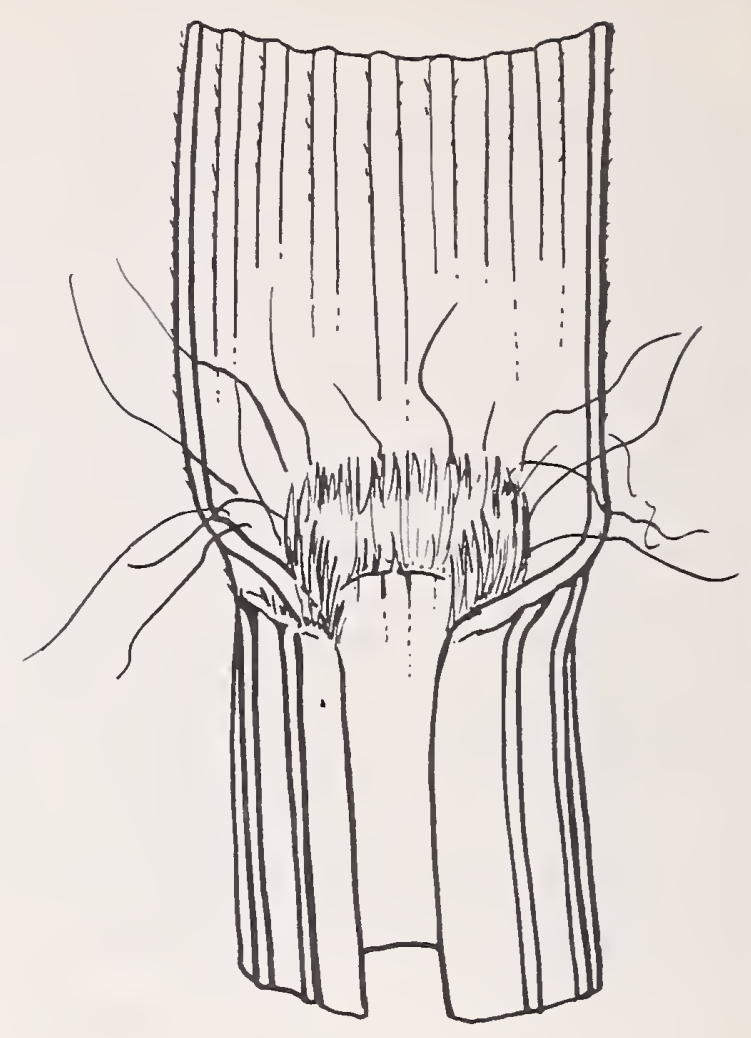

10. Aristida glauca (Nees) Walp. BLUE THREE-AWN

Erect, medium bunchgrass. VERNATION - Clasped. BLADES - Rolled, narrow, pointed; usually glabrous, stiff; veins and ribs indistinct; margin smooth; midrib prominent; $1 / 2 \mathrm{~mm}$. wide, $15-25 \mathrm{~cm}$. long. AURICLE - None. LIGULE - A collar, small 1/4-1/2 mm., truncate-ciliate. COLLAR Ciliate front margin divided. SHEATH Round, glabrous. NODES - Glabrous. INTERNODES - Glabrous. ROOTS - Fibrous. CULM - Round, usually branched. OUTSTANDING CHARACTERS - Blades rolled, narrow, long, pointed. Culms usually branched. Ligule ciliate, short. Awns 2-4 $\mathrm{cm}$. long.

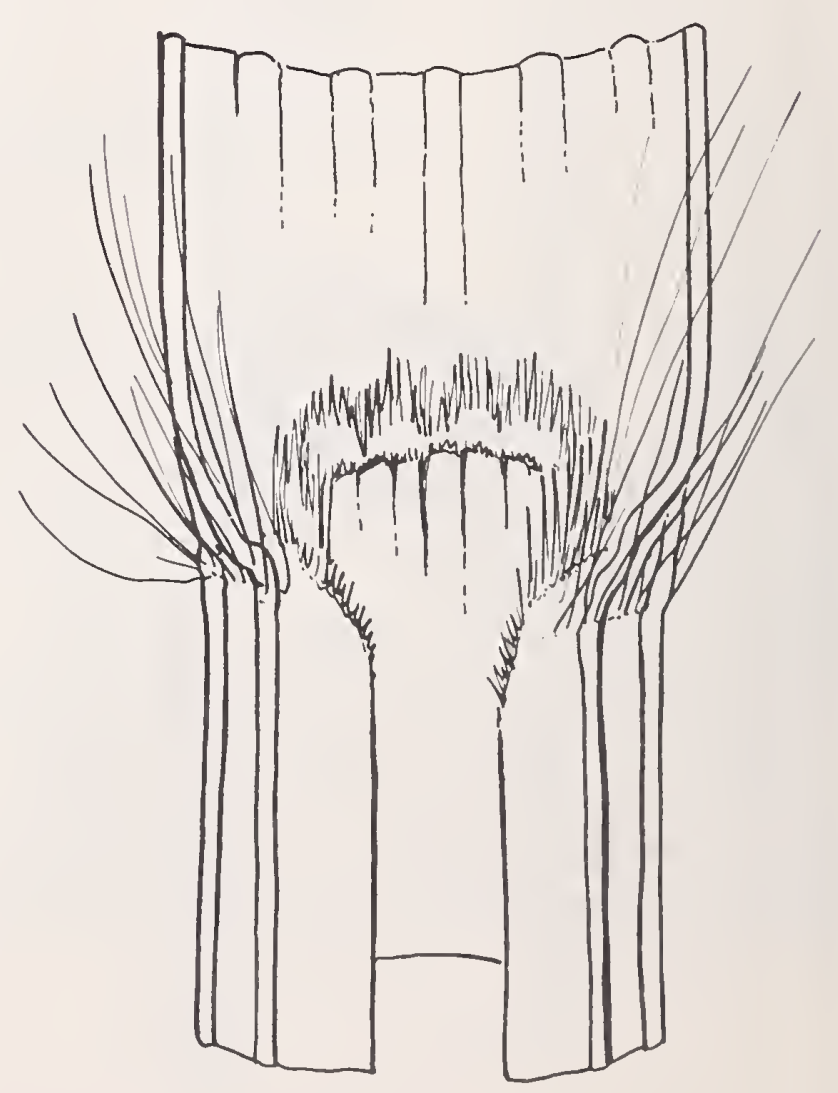


11. Aristida longiseta Steud. RED THREE-AWN

Erect, small bunchgrass. VERNATION - Clasped. BLADES - Rolled, narrow, long; rough ventrally, stiff; ribs not prominent; margin toothed; midrib not prominent; 1 mm. wide, 10-20 cm. long. AURICLE None. LIGULE - Hairy, tufted, 1-2 mm. COLLAR - Hairy margin, divided. SHEATH - Round, veined, glabrous, papery margin. NODES - Glabrous. INTERNODES - Glabrous. ROOTS - Fibrous. CULM - Round, not branched. OUTSTANDING CHARACTERS - Blades rolled, narrow, long. Ligule hairy, tufted, 1-2 mm. Awn 5-8 cm. long.

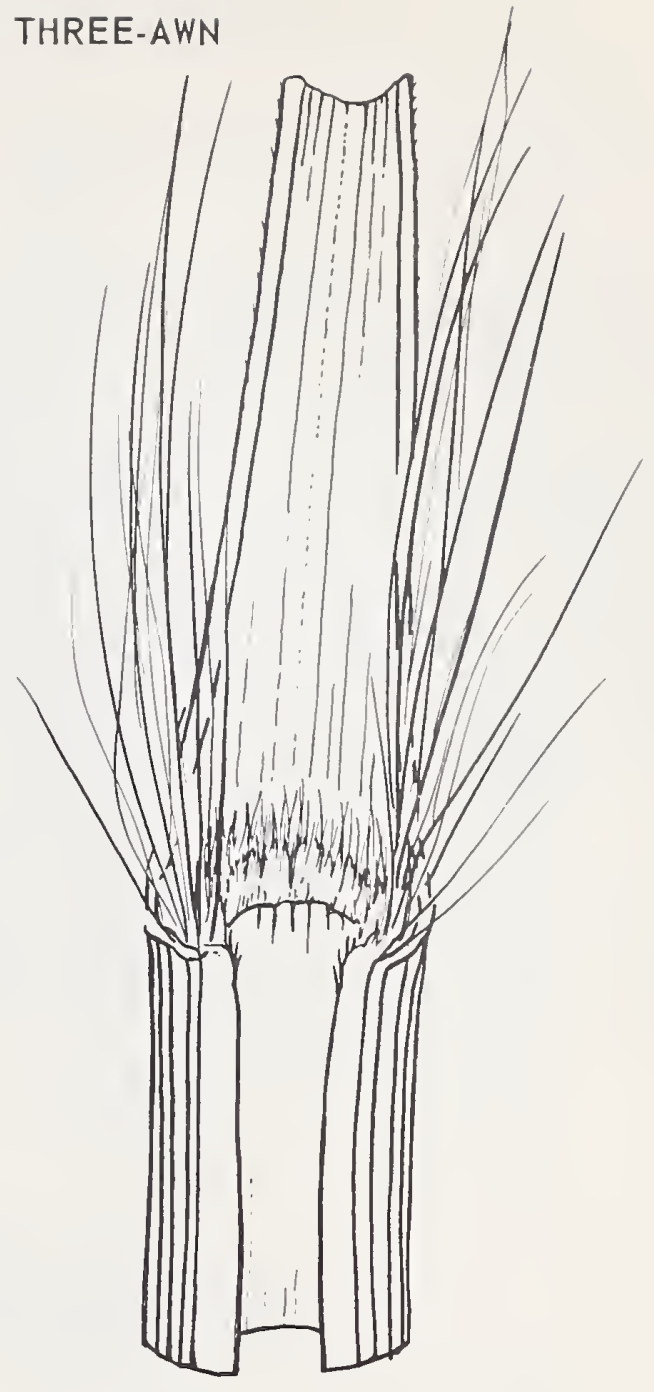

12. Aristida purpurea Nutt. PURPLE THREE-AWN

Erect, small bunchgrass. VERNATION - Folded. BLADES - Rolled, curved, pointed; rough dorsally and ventrally, occasionally hairy; ribs indistinct; margin occasionally hairy, $1 / 2 \mathrm{~mm}$. wide, $2-8 \mathrm{~cm}$. long. AURICLE - None. LIGULE - Hairy, 1-1 1/2 mm. COLLAR - Hairy margin, bearded. SHEATH - Glabrous, round. NODES - Glabrous. INTERNODES - Glabrous. ROOTS - Fibrous. CULM - Elliptical, not branched. OUTSTANDING CHARACTERS - Blades rolled, thread-like, curved, short. Collar bearded. Ligule conspicuous hairs. Awns purple, $2-5 \mathrm{~cm}$.

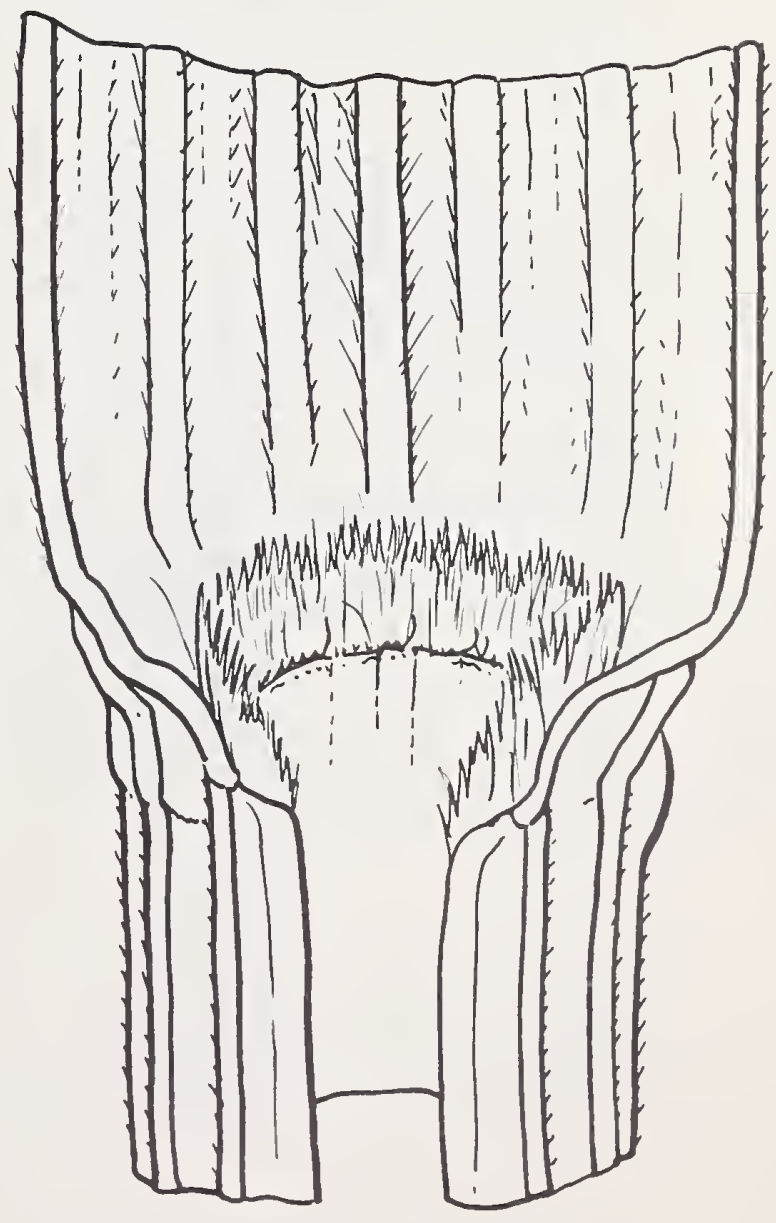


Erect, small bunchgrass. VERNATION - Folded. BLADES - Flat at base, tip rolled; rough, occasional hairs dorsally; veins each side of midrib, 2; ribs prominent ventrally and dorsally; margin toothed; midrib not prominent; 2 mm. wide, 5-20 $\mathrm{cm}$. long. AURICLE - None. LIGULE Hairy, small $1 \mathrm{~mm}$. COLLAR - Hairy margin, $1 \mathrm{~mm}$, divided. SHEATH - Glabrous, papery margin. NODES - Glabrous. INTERNODES - Glabrous. ROOTS - Fibrous. CULM - Elliptical, occasionally branched. OUTSTANDING CHARACTERS Blades flat at base, narrow, long, ribbed. Ligule hairy, small. Collar hairy margin. Awns, 1 long and 2 short.

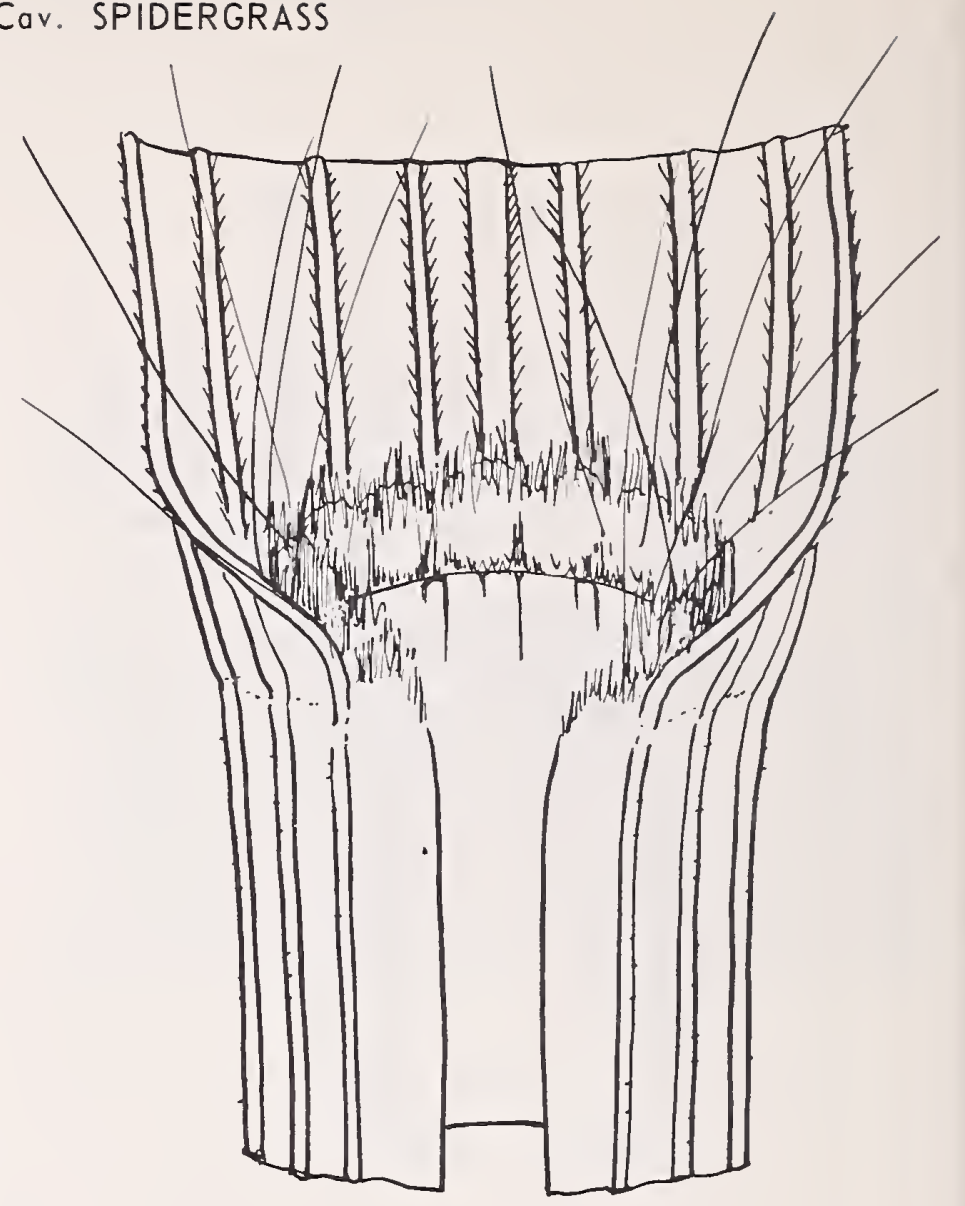

14. Blepharoncuron tricholepis (Torr.) Nash. PINE DROPSEED

Erect, small bunchgrass. VERNATION - Folded. BLADES - Usually rolled, narrow, pointed; rough, stiff, ribs indistinct; margin toothed; midrib not prominent; $1 \mathrm{~mm}$. wide, 5-10 cm. long. AURICLE None. LIGULE - Membranous, very small, $1 \mathrm{~mm}$., truncate-entire. COLLAR - Glabrous. SHEATH - Elliptical, glabrous, veined. NODES - Glabrous. INTERNODES - Glabrous. ROOTS - Fibrous. CULM Round, not branched. OUTSTANDING CHARACTERS - Blades long, narrow, usually rolled. Ligule small, truncateentire. Few basal blades.

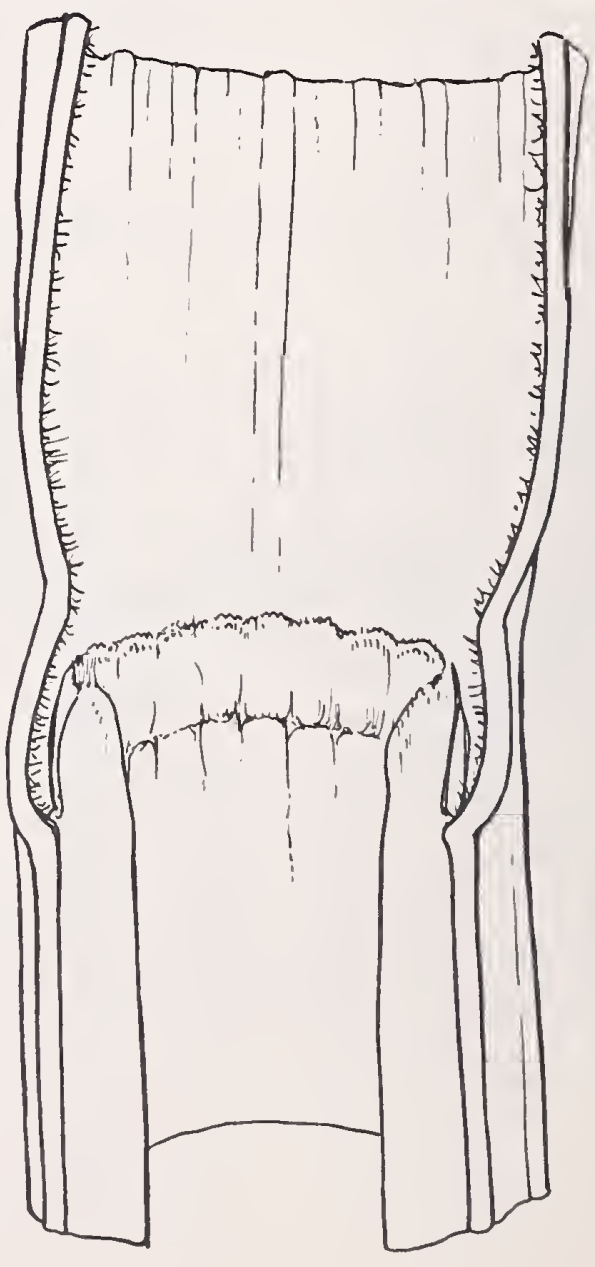


Annual semi-erect, small bunchgrass. VERNATION - Curled. BLADES - Flat, erect, narrow, pointed; blade hairy near collar; veins each side of midrib, 1-2; ribs indistinct; margin with grandular hairs near ligule, 2-4 mm.; midrib prominent ventrally; 1-2 $\mathrm{mm}$. wide, $2-7 \mathrm{~cm}$. long. AURICLE - None. LIGULE - Hairy, small $1 / 2 \mathrm{~mm}$. COLLAR - Glabrous. SHEATH Glabrous, veined, elliptical. NODES - Glabrous. INTERNODES - Glabrous. ROOTS Fibrous. CULM - Elliptical, branched. OUTSTANDING CHARACTERS - Annual. Ligule hairy. Blade margin glandular hairs. Stems branched. Awns short.

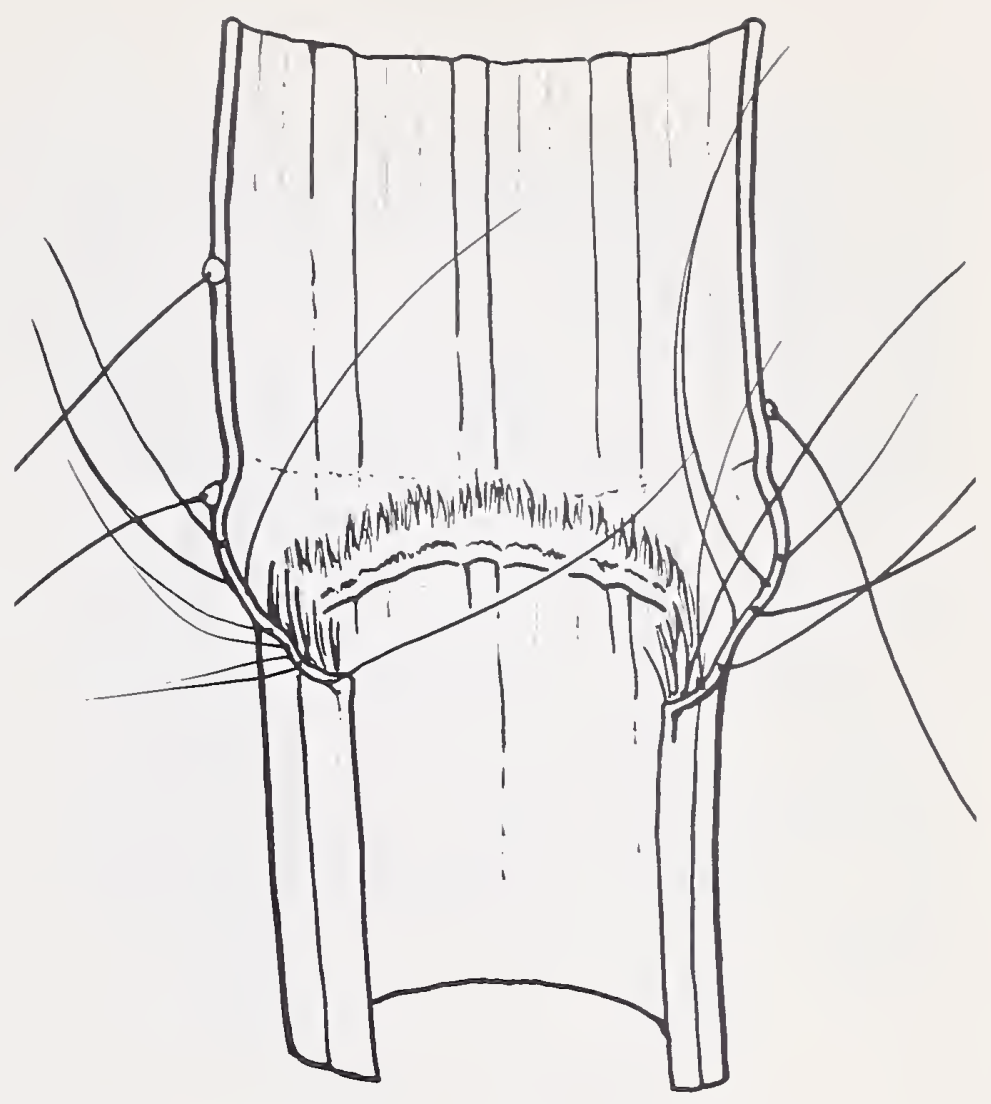

16. Bouteloua barbata Lag. SIXWEEKS GRAMA

Annual, erect, small bunchgrass. VERNATION - Curled. BLADES - Flat, narrow, short; glabrous; veins each side of midrib, 2; ribs not prominent; margin glabrous; midrib not prominent; $1 \mathrm{~mm}$. wide, 2-4 cm. long. AURICLE - None. LIGULE Hairy, small $1 \mathrm{~mm}$. COLLAR - Glabrous. SHEATH - Glabrous, veined, margin papery. NODES - Glahrous. INTERNODES Glabrous. ROOTS - Fibrous. CULM Branched, round. OUTSTANDING CHARACTERS - Annual. Roots superficial, blows away at maturity. Ligule hairy. Blades flat, narrow, short. Sheath margin papery. Awns short.

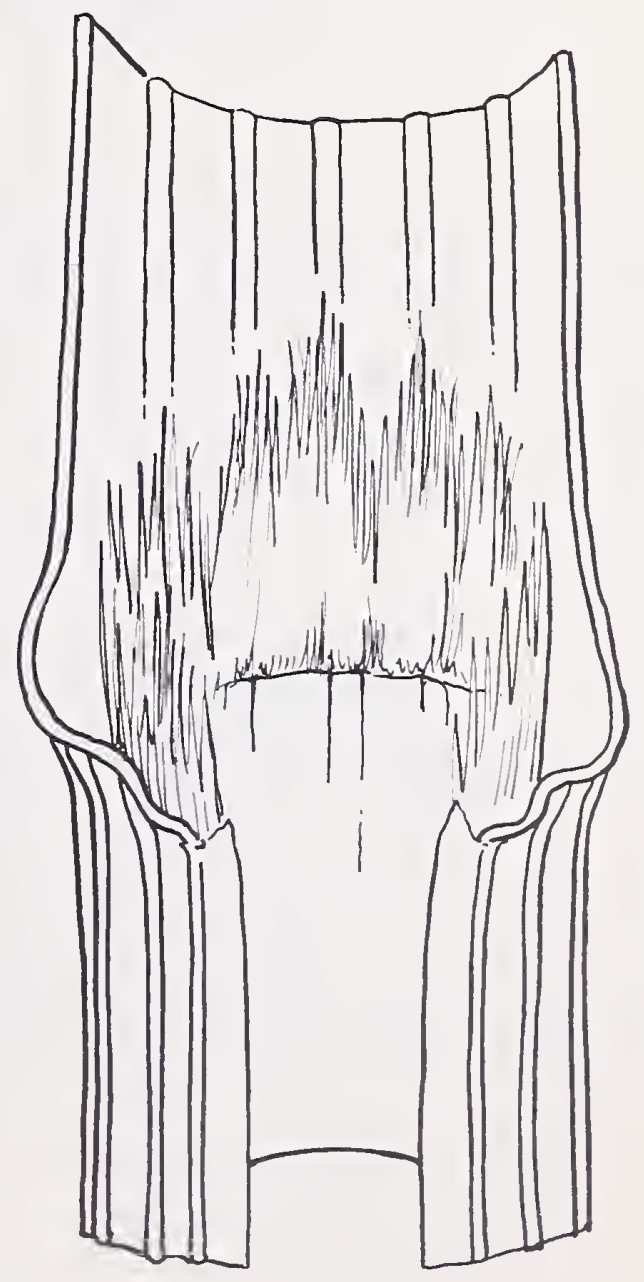


Erect, small bunchgrass. VERNATION - Clasped. BLADES - Flat, drooping, narrow, pointed; occasionally hairy ventrally and dorsally; veins each side of midrib, 4; ribs numerous, prominent ventrally and dorsally; margin with glandular hairs, midrib prominent ventrally; $2 \mathrm{~mm}$. wide, 5-15 cm. long. LIGULE - Hairy, $1 / 4 \mathrm{~mm}$. COLLAR - Hairy dorsally, margin divided. SHEATH - Round, veined, glabrous. NODES - Glabrous. INTERNODES - Glabrous. ROOTS - Fibrous. CULM - Round, not branched。OUTSTANDING CHARACTERS Glandular hairs on blade margin, dorsally and occasionally ventrally. Ligule hairy, small 1/4 nm. Spikelet villous, short and broad.

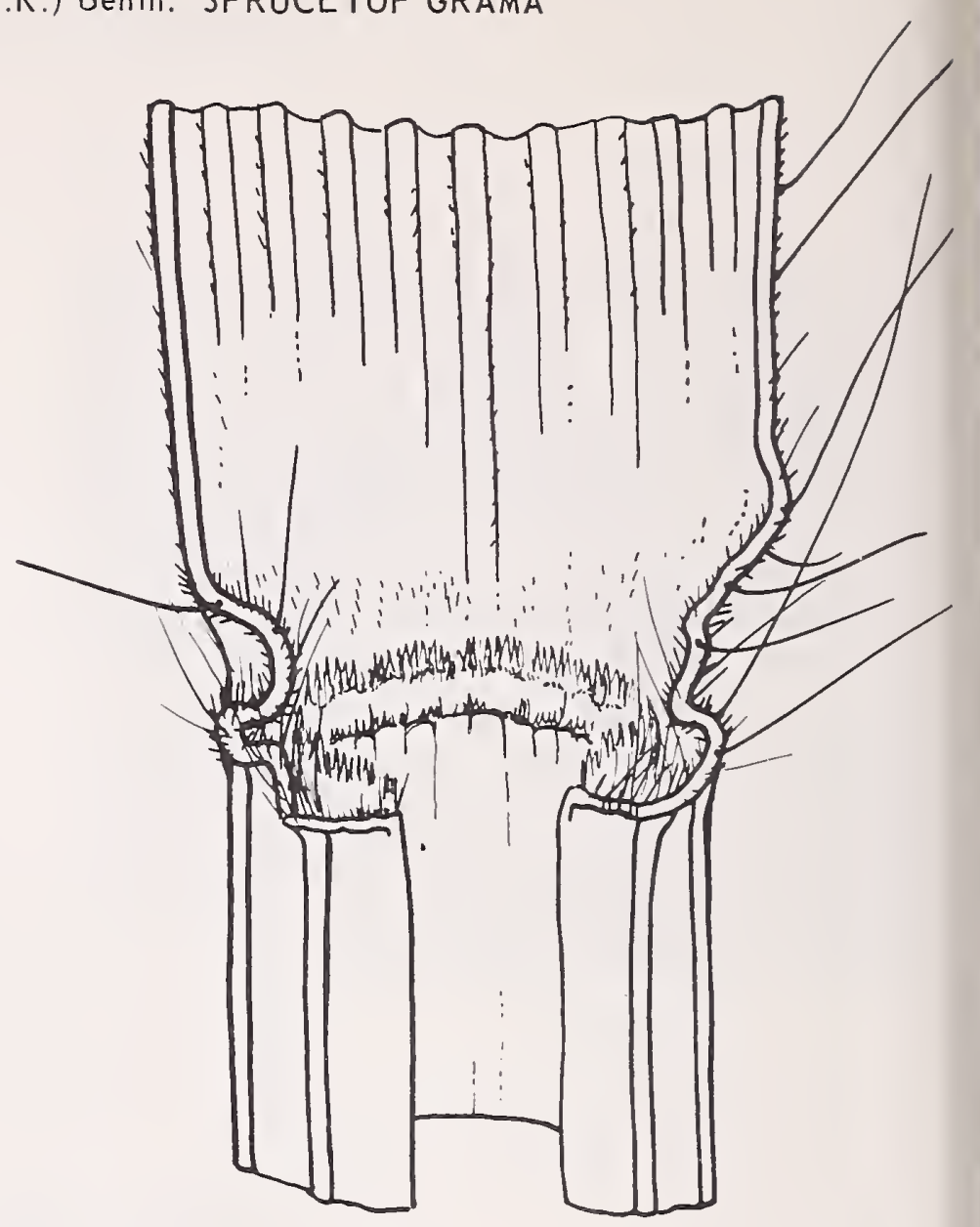

18. Bouleloua curtipendula (Michx.) Torr. SIDEOATS GRAMA
Erect, large bunchgrass. VERNATION - Curled. BLADES - Flat, long, drooping, narrow, pointed; rough dorsally; veins each side of midrib, 2-3; ribs not prominent; margin toothed, glandular hairs; midrib not prominent; $2-3 \mathrm{~mm}$. wide, 5-20 $\mathrm{cm}$. long, AURICLE - None. LIGULE Membranous, small $1 \mathrm{~mm}$, truncatelacerate. COLLAR - Hairy margin and occasionally glandular. SHEATH - Glabrous, veined, papery margin. NODES Glabrous. INTERNODES - Glabrous. ROOTS - Short rhizomes. CULM - Elliptical to round occasionally branched. OUTSTANDING CHARACTERS - Blade and collar margins with conspicuous glandular hairs. Rachis zigzag. Mature plants reddish-brown.

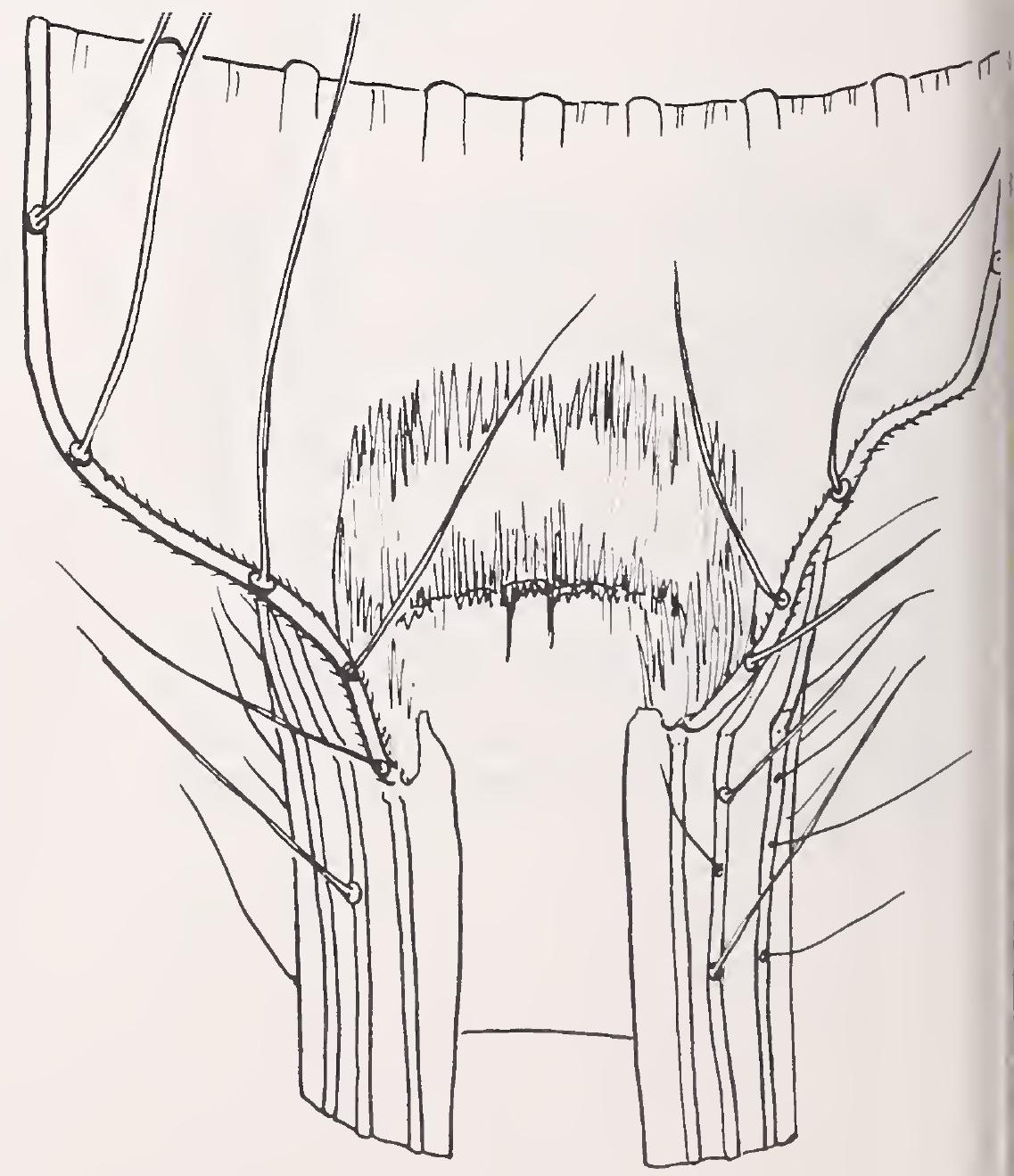


Erect to generally decumbent. VERNATION - Curled. BLADES - Flat, narrow, drooping, pointed; soft, hairy ventrally; veins each side of midrib, usually 2 ; rib not prominent; margin glandular hairs, 3-5 mm., midrib not prominent; 1-2 mm. wide, 3-8 cm. long. AURICLE - None. LIGUI..E Hairy, small, 1/2 mm. COLLAR - Glabrous or with scattered hairs. SHEATH Round, glabrous, NODES - Sparsely pubescent to glabrous. INTERNODES - Woolly. ROOTS - Fibrous. CULM - Round, branch at node, woolly. OUTSTANDING CHARACTERS - Blade margin glandular hairs. Internodes canescent. Culm roots at nodes and produces new plants. Old plants surrounded by new plants connected with stolons. Lower stems remain green year long.

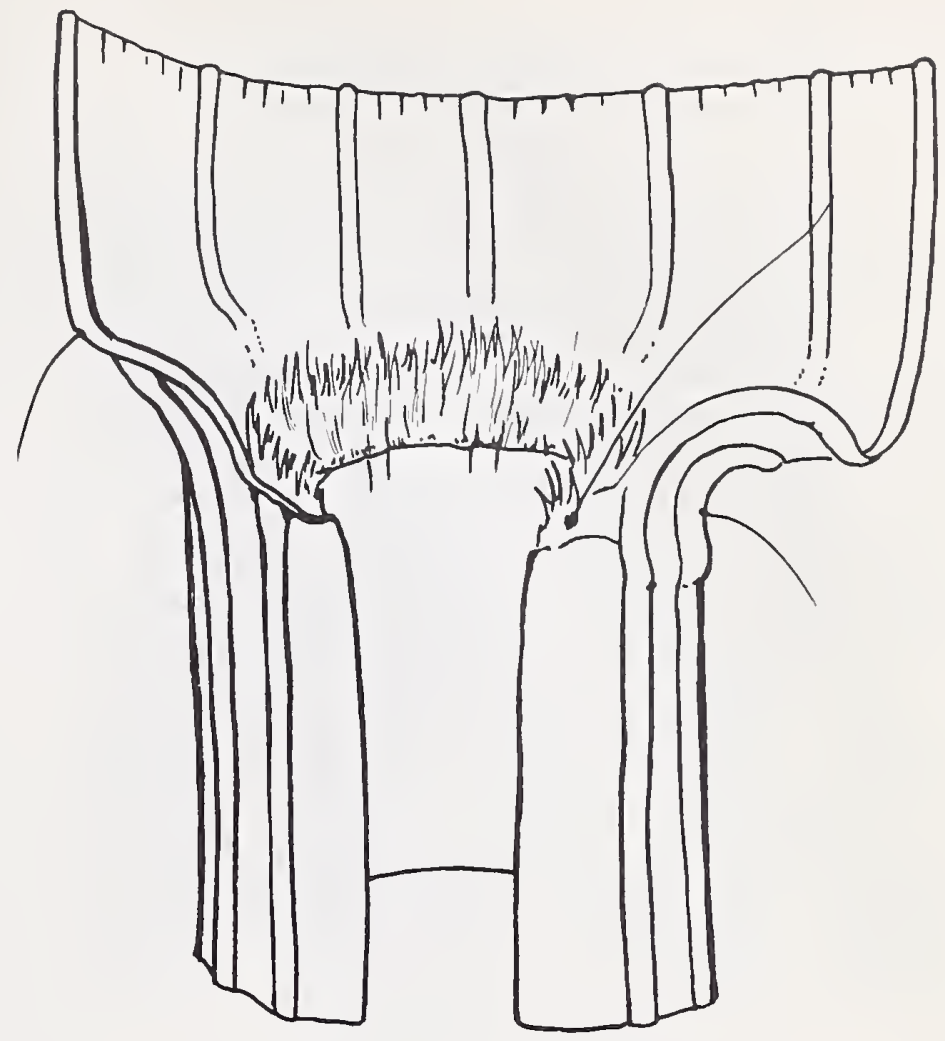

20. Bouteloua filiformls (Fourn.) Griffiths. SLENDER GRAMA

Erect, small bunchgrass. VERNATION - Curled. BLADES - Flat, narrow, pointed; margin rough, glandular hairs; veins each side of midrib, 2-3; ribs prominent (with lens) ventrally and dorsally, midrib prominent ventrally; 1-2 $\mathrm{mm}$. wide, $5-8 \mathrm{~cm}$. long. AURICLE - None. LIGULE - Hairy, small 1/2 mm., ciliate. COLLAR - Hairy margin. SHEATH - Glabrous, papery margin. NODES - Glabrous. INTERNODES Glabrous. ROOTS - Fibrous. CULM - Elliptical, not branched. OUTSTANDING CHARACTERS - Blade margin glandular hairs. Ligule ciliate, small $1 / 2 \mathrm{~mm}$. Blades narrow, long.

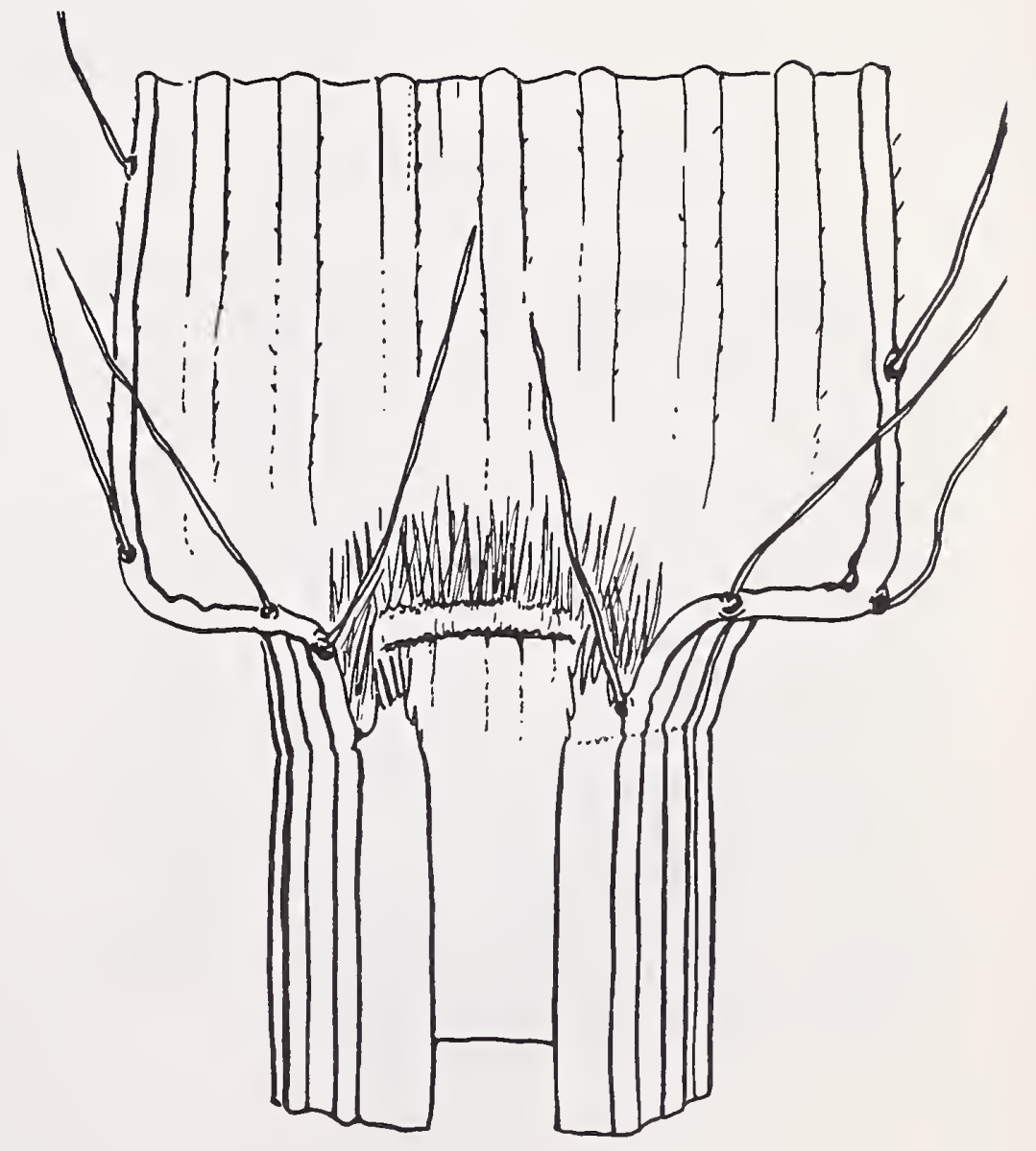


Erect, open sod or mat. VERNATION Clasped. BLADES - Flat, drooping, narrow, pointed; rough ventrally, veins each side of midrib usually 2; veins prominent ventrally and dorsally, margin toothed; sparsely pilose below; 2-3 mm. wide, $5-15$ cm. long. AURICLE - None. LIGULE Small 1/2 mm. mostly of hairs. COLLAR Glabrous (smooth, not pubescent or hairy). SHEATH - Round, glabrous, veined. NODES - Glabrous. INTERNODES - Glabrous. ROOTS - Fibrous, short rhizomes. CULM - Round, not branched. OUTSTANDING CHARACTERS - Blade in bud clasped. Blades flat, narrow, long drooping, glabrous. Usually semi-sod. Old growth blades curled.

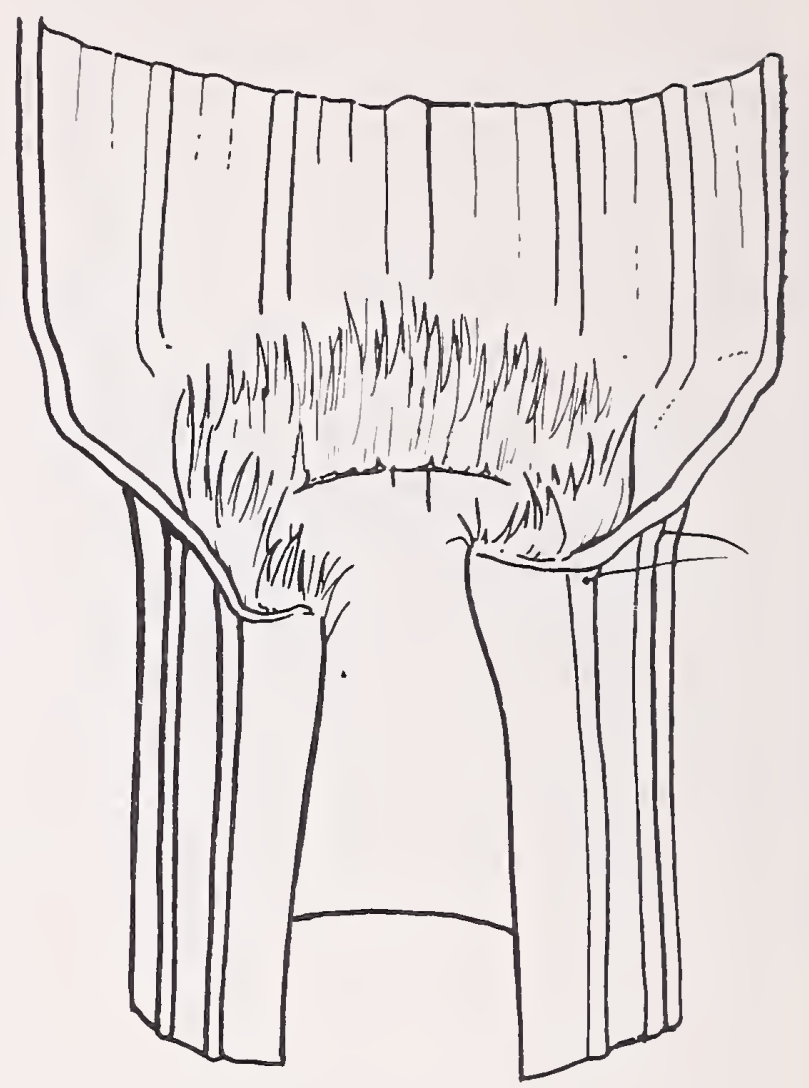

22. Boutcloua hirsuta Lag. HAIRY GRAMA

Erect, small bunchgrass. VERNATION - Clasped. BLADES - Flat, drooping, narrow, pointed; rough, hairy dorsally; veins each side of midrib, 3-4 ribs indistinct; margin glandular hairs: midrib not prominent; 1-3 mm. wide, 3-10 cm. long. AURICLE - None. LIGULE - Membranous, small $1 / 4 \mathrm{~mm}$. with occasional marginal hairs; truncate ciliate. COLLAR - Hairy with glandular marginal hairs. SHEATH Veined, round with papery margin. NODES - Glabrous. INTERNODES - Glabrous. ROOTS - Fibrous. CULM - Not branched, round. OUTSTANDING CHARACTERS Blades margin with glandular hairs. Rachis extends beyond terminal spikelet. Collar margin with glandular hairs.

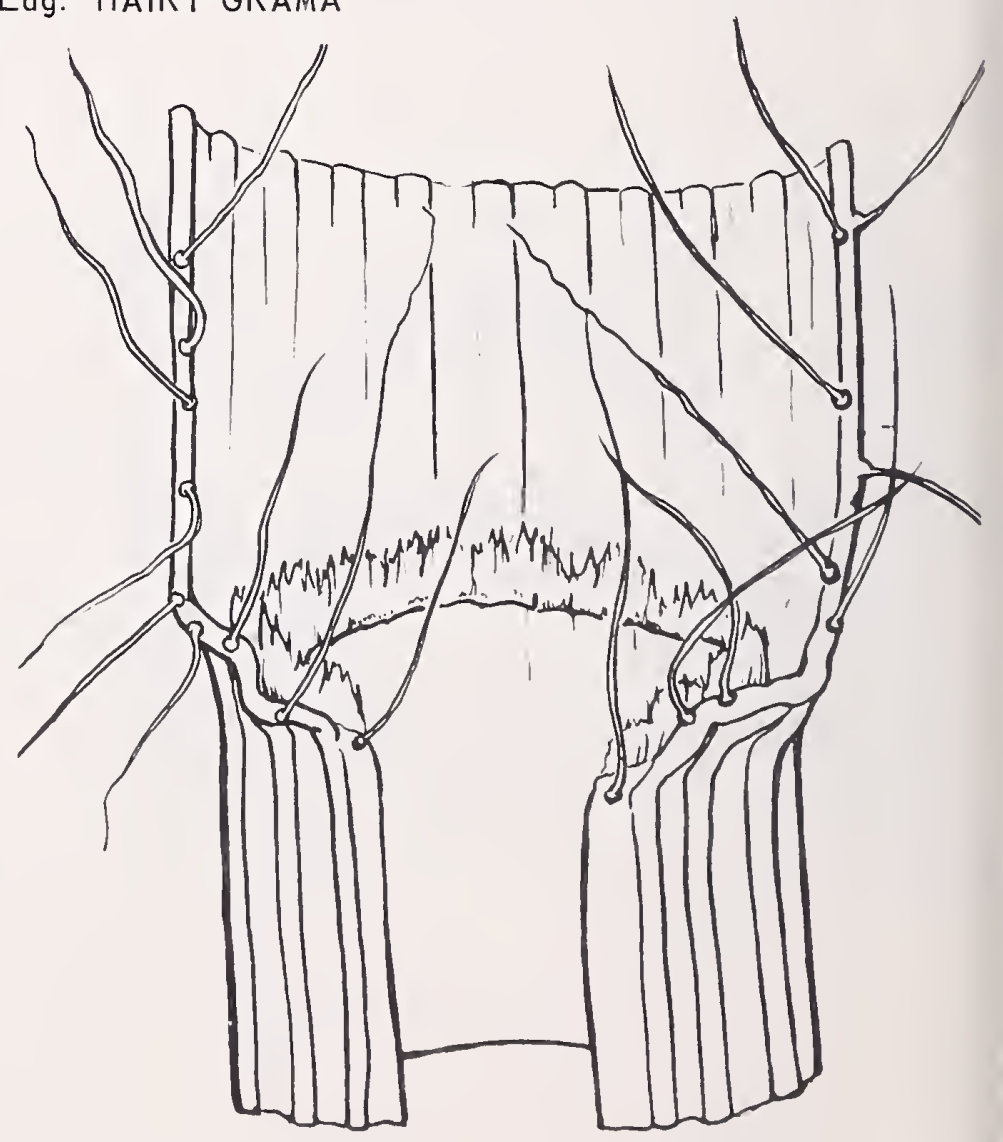


Erect to semi-erect, small bunchgrass. VERNATION - Curled. BLADES - Flat, drooping, narrow, long, pointed; rough, hairy ventrally and dorsally; veins each side of midrib 2; ribs numerous, not prominent; margin glandular hairs, toothed; midrib prominent dorsally; $2 \mathrm{~mm}$. wide, 5-15 cm. long. AURICLE - None. LIGULE - Hairy, tufted, 2-4 mm. COLLAR - Hairy margin, divided. SHEATH - Glabrous, papery margin. NODES - Glabrous. INTERNODES - Glabrous. ROOTS - Fibrous. CULM - Round, not branched. OUTSTANDING CHARACTERS - Glandular hairs on blade margin, dorsally and occasionally ventrally. Ligule and collar hairy. Spikelets $3-8$, slender.

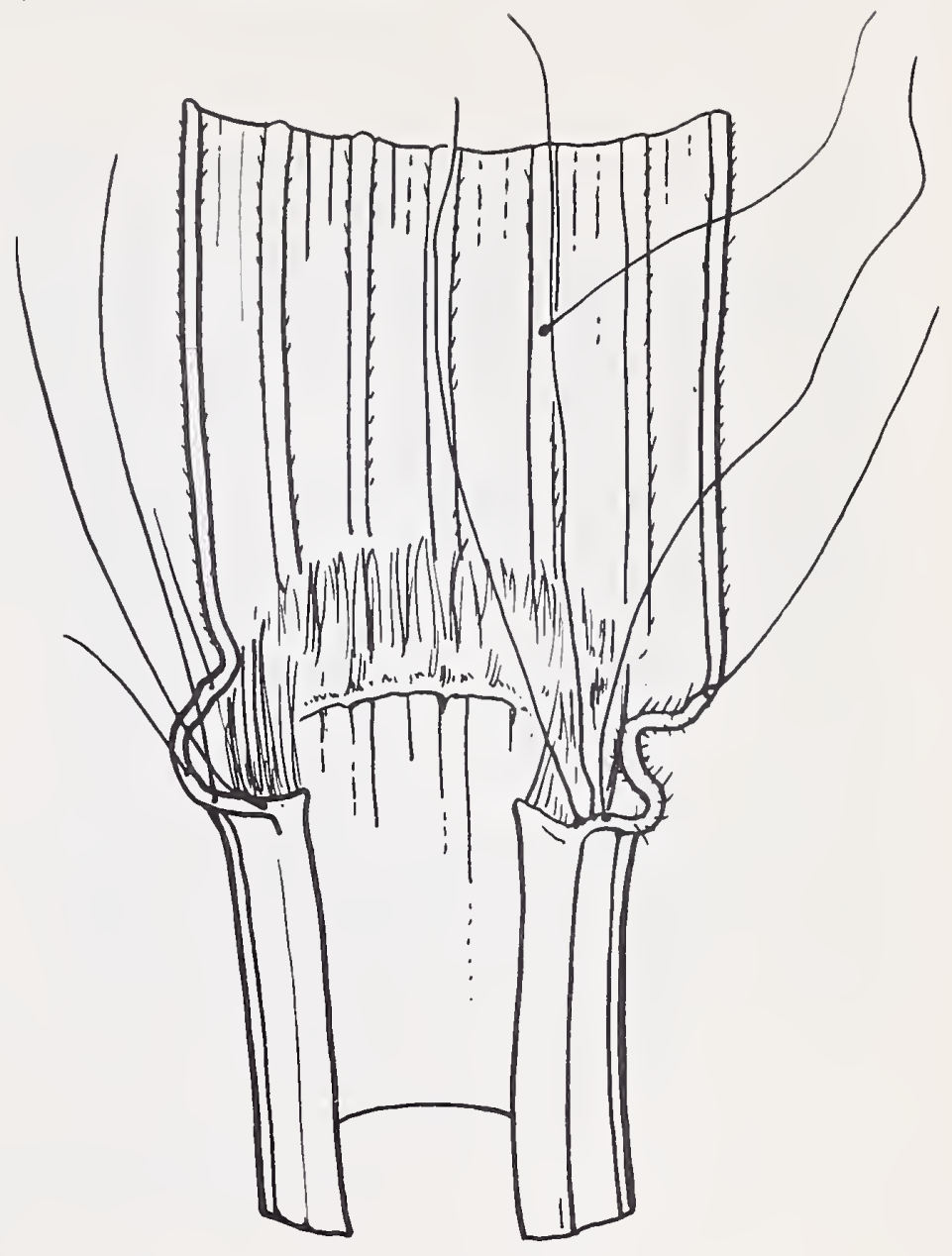

24. Bromus anomalus Rupr. NODDING BROME

Erect, small bunchgrass. VERNATION - Curled. BLADES - F'lat, wide, long, drooping; occasionally hairy dorsally, 2-4 mm.; veins each side of midrib, 3 ; ribs prominent ventrally and dorsally; margin toothed; midrib prominent dorsally and ventrally; 2-8 $\mathrm{mm}$. wide, $15-40 \mathrm{~cm}$. long. AURICLE - None. LIGULE - Membranous, small $1 / 2 \mathrm{~mm}$, truncate-toothed. COLLAR - Glabrous, divided. SHEATH Ribbed, generally closed, glabrous to hairy on margin, the lower sheaths sometimes retrorsely hispid. NODES - Glabrous. INTERNODES - Glabrous. ROOTS - Fibrous. CULM - Elliptical, not branched. OUTSTANDING CHARACTERS - Sheath usually closed completely. Blades flat and sheath with prominent ribs. Blades wide, long, drooping. Ligule membranous, $1 / 2 \mathrm{~mm}$. truncate-toothed. Blade midrib prominent dorsally and ventrally.

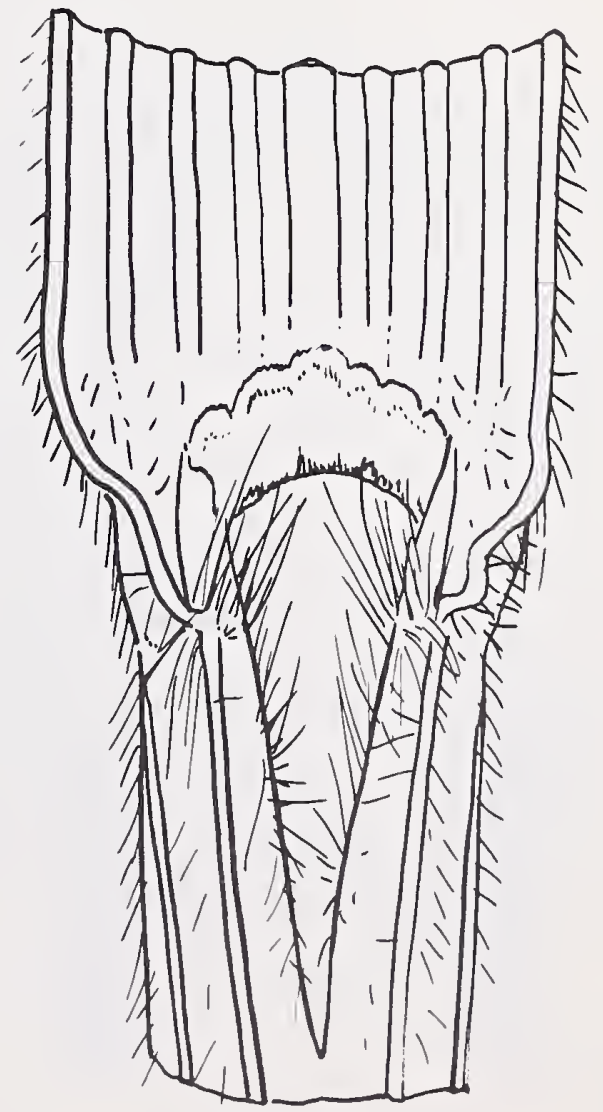


Erect, annual. VERNATION - Curled. BLADES - Flat, wide, long; hairy ventrally and dorsally; veins each side of midrib, 2-3; ribs prominent ventrally and dorsally; margin hairy; midrib prominent dorsally; 4-6 $\mathrm{mm}$. wide, $10-20 \mathrm{~cm}$. long. AURICLE - None. LIGULE - Membranous, hyaline, large 2-5 mm., obtuse-lacerate. COLLAR - Glabrous, divided. SHEATH Closed, hairy. NODES - Glabrous. INTERNODES - Glabrous, veined. ROOTS Fibrous, annual. CULM - Round, not branched. OUTSTANDING CHARACTERS Annual. Sheath closed. Blades hairy. Ligule hyaline, large 2-5 mm., obtuse-lacerate. Awns, 5-10 $\mathrm{mm}$.

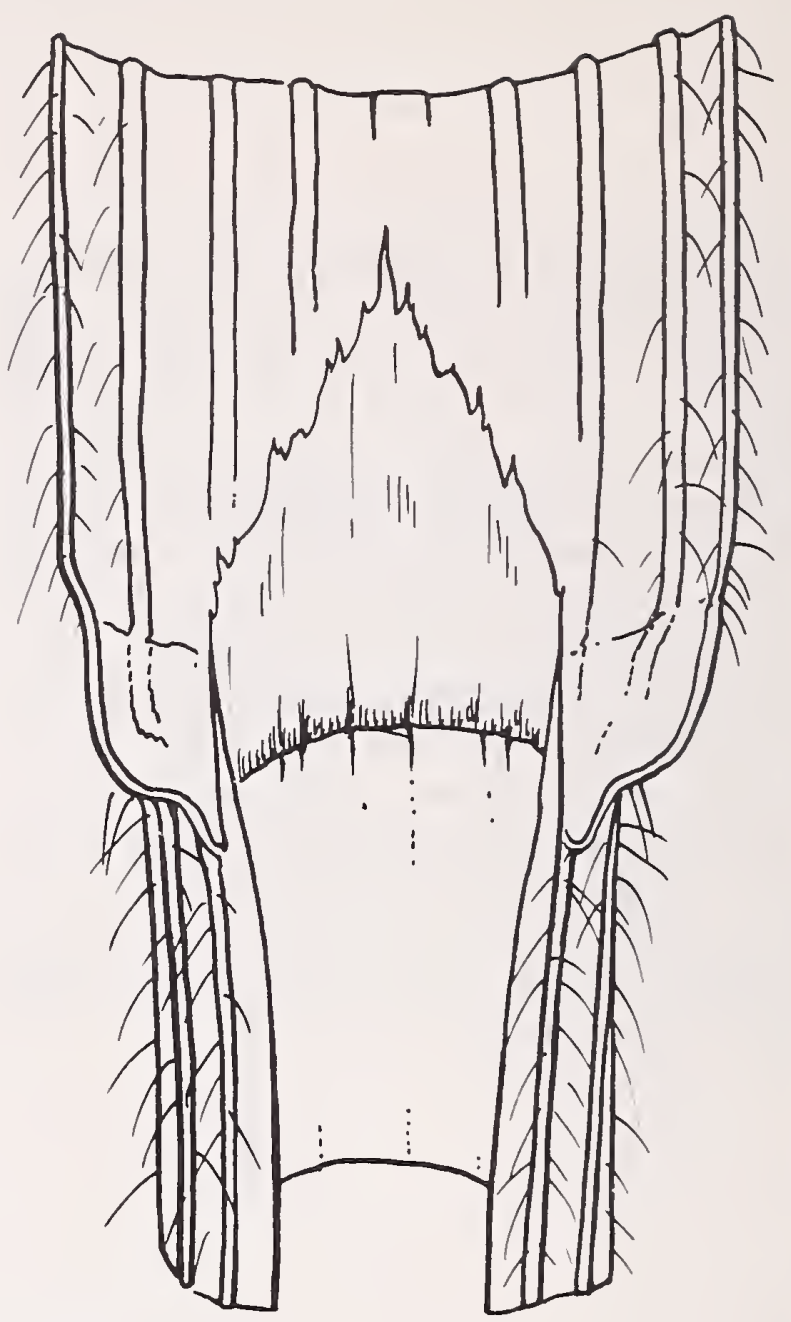

26. Bromus ciliatus L. FRINGED BROME

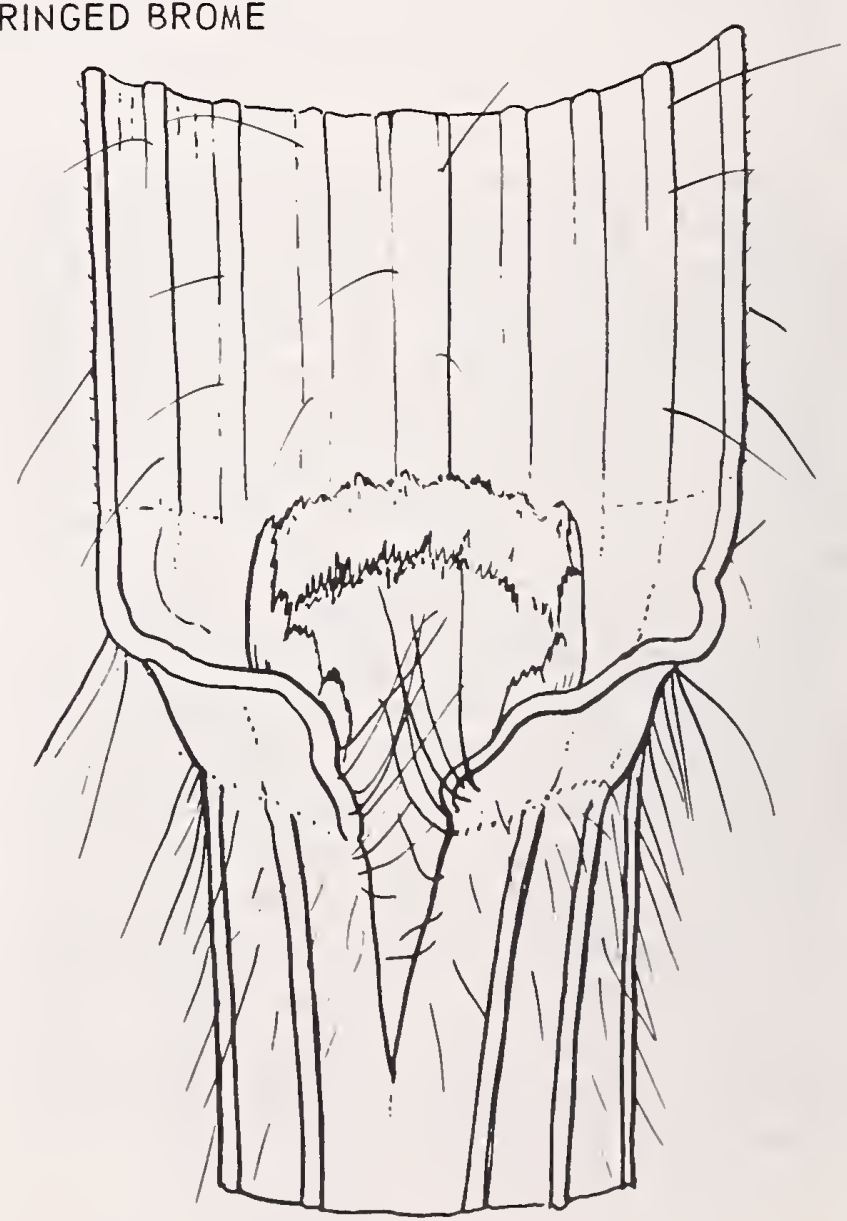


Large erect bunchgrass. VERNATION - Curled. BLADES - Flat, wide, drooping, usually a $W$ on blade; rough ventrally and dorsally; veins each side of midrib, 5-6; rib prominent ventrally and dorsally; midrib prominent dorsally; 4-5 mm. wide, 10-20 cm. long. AURICLE - None. LIGULE - Membranous, small 1/2 mm. COLLAR Glabrous, divided. SHEATH - Closed, sometimes hairy, veined, round, papery margin. NODES - Occasionally pubescent. INTERNODES - Glabrous, veined. ROOTS - Short rhizomes. CULM - Round, not branched, large. OUTSTANDING CHARACTERS - Sheath closed. The letter W or M on blades. Blades large, flat, long ribbed.

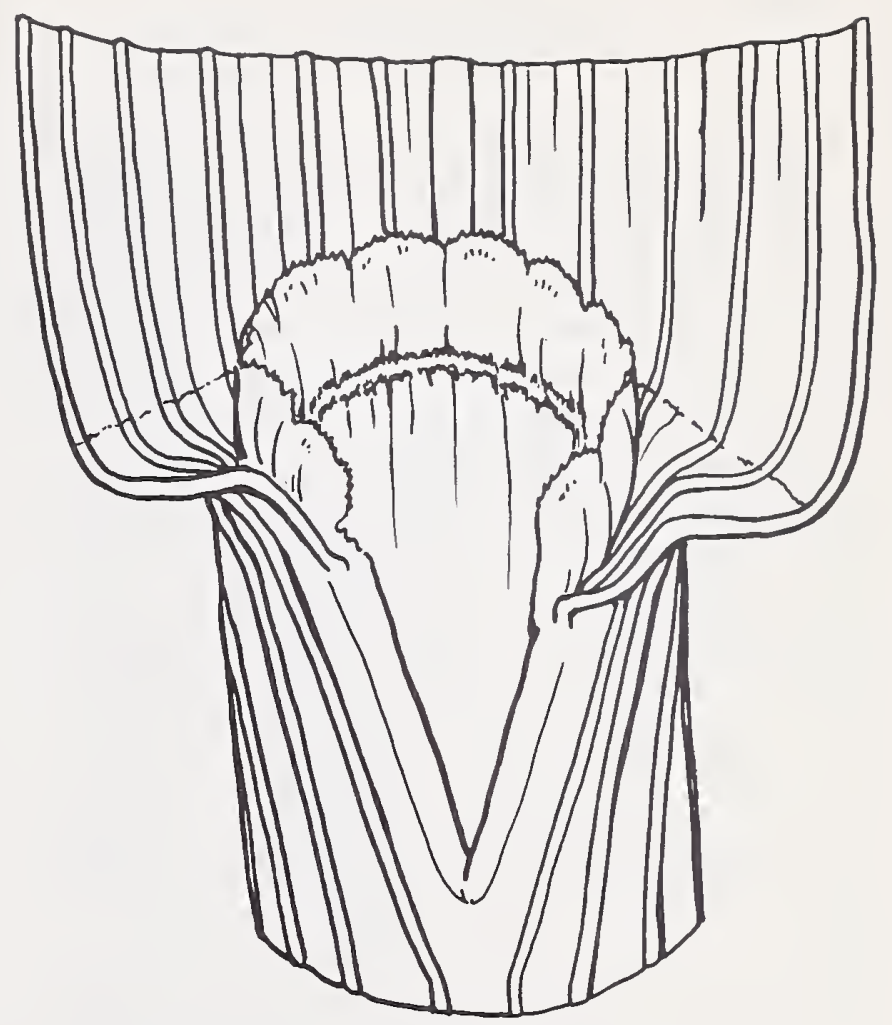

28. Bromus rubens L. RED BROME

Erect to semi-decumbent, anmual. VERNATION - Curled. BLADES - Flat, pointed; hairy dorsally and ventrally; veins each side midrib, 2-3; ribs prominent ventrally and dorsally; blade margin smooth; midrib prominent, 1-2 mm. wide, $2-6 \mathrm{~cm}$. long. AURICLE - None. LIGULE - Membranous, $1 / 2-3 \mathrm{~mm}$. long, acute-lacerate. COLLAR - Smooth. SHEATH - Pubescent, papery margin, round, usually closed. NODES - Glabrous. INTERNODES - Occasionally puberulent near nodes. ROOTS Fibrous, weak. CULM - Not branched, round. OUTSTANDING CHARACTERS Winter anmual. Blades short, narrow, flat. Midrib prominent ventrally. Ligule acutelacerate. Awns 1/2-1 cm. Head at maturity reddish color.

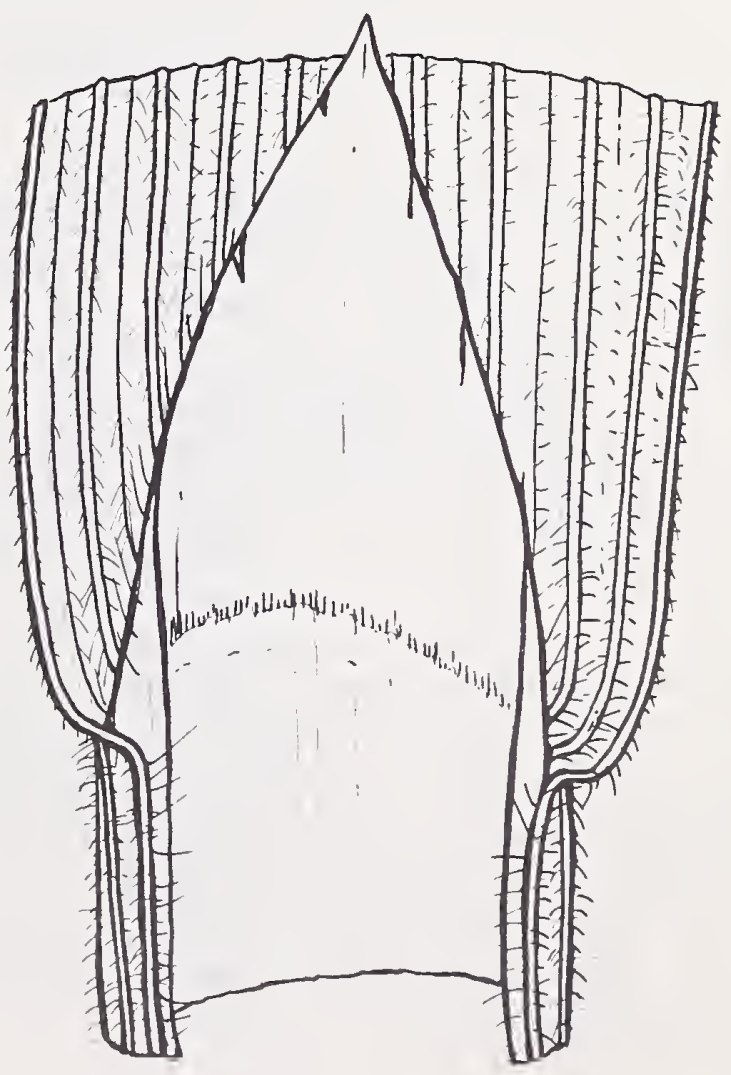


Semi-erect, small bunchgrass, annual. Heads open, drooping. VERNATION Curled. BLADES - Flat twisted, drooping, blunt pointed; hairy ventrally and dorsally, soft; veins each side of midrib, $2-3$; ribs indistinct; margin hairy; midrib prominent ventrally; 2-4 mm. wide, $5-10 \mathrm{~cm}$. long. AURICLE - None. LIGULE - Membranous, collar-like, small 1-2 mm., obtuse-lacerate, paper-like. COLLAR - Smooth, divided. SHEATH - Woolly, round, closed. NODES Glabrous, dark in color. INTERNODES Glabrous. ROOTS - Shallow, fibrous. CULM - Round, not branched. OUTSTANDING CHARACTERS - Awn, 1-2 cm. Winter annual. Fire hazard. Sheath growth together. Ligule very thin, obtuse-lacerate. Woolly over most of plant, soft to touch. In northern climates grows in spring and fall. Excellent for lambing.

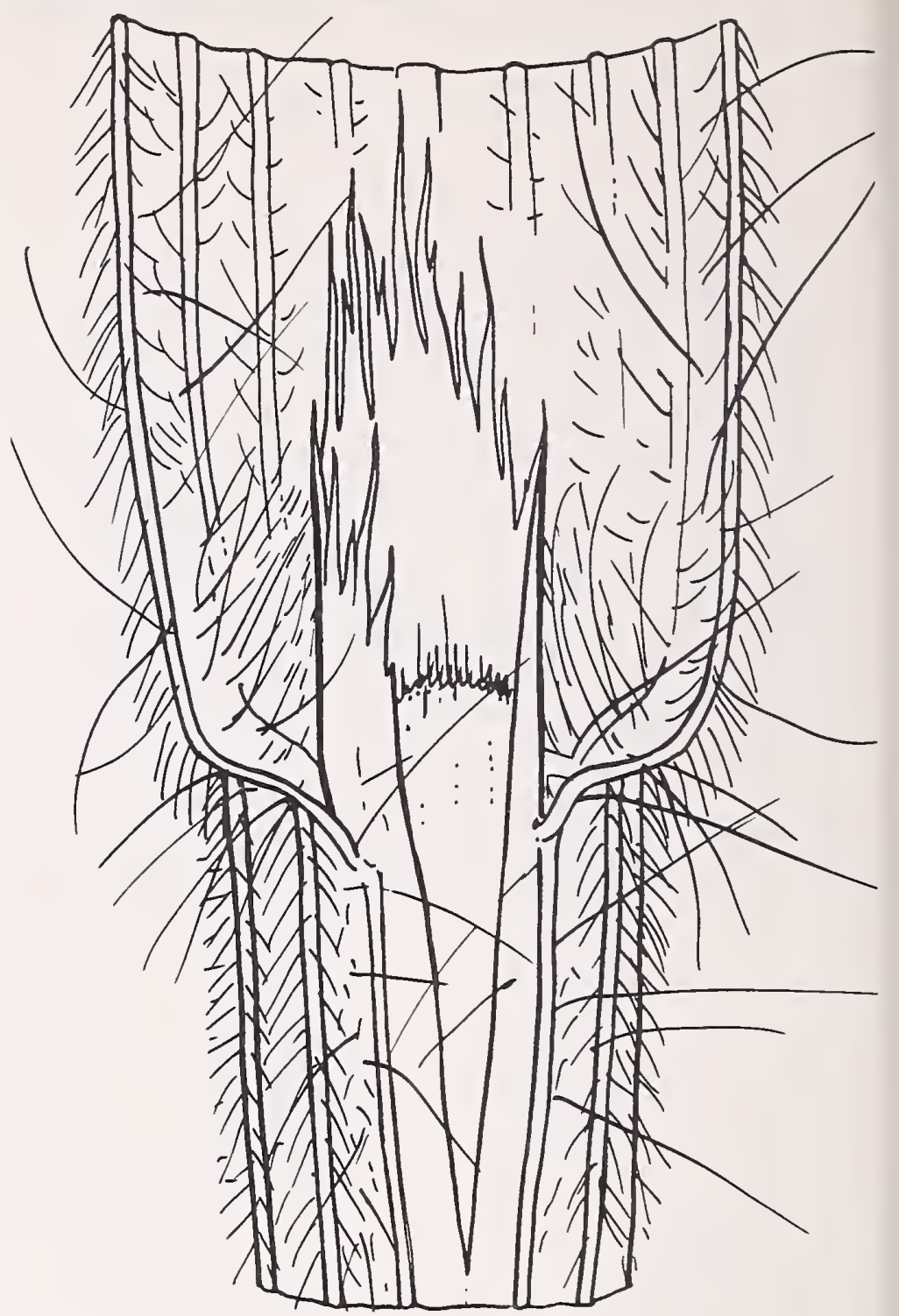

30. Chloris virgata Swartz. FEATIHER FINGERGRASS

Semi-erect, annual small bunchgrass. VERNATION - Curled. BLADES - Flat, wide, long; occasionally glandular hairs dorsally near base, 2-3 mm.; veins each side of midrib, 3 ; ribs numerous, not prominent; margin often straw colored; midrib prominent ventrally; $3-6 \mathrm{~mm}$. wide, 8-15 cm. long. AURICLE - None. LIGULE Membranous, small, $1 \mathrm{~mm}$., truncateciliate. COLLAR - Glabrous. SHEATH Glabrous, hyaline margin. NODES - Glabrous. INTERNODES - Glabrous. ROOTS Fibrous, annual. CULM - Elliptical, branched at base. OUTSTANDING CHARACTERS - Annual. Blades flat, wide, occasionally glandular hairs dorsally. Ligule $1 \mathrm{~mm}$, truncate-ciliate. Blade midrib prominent ventrally.

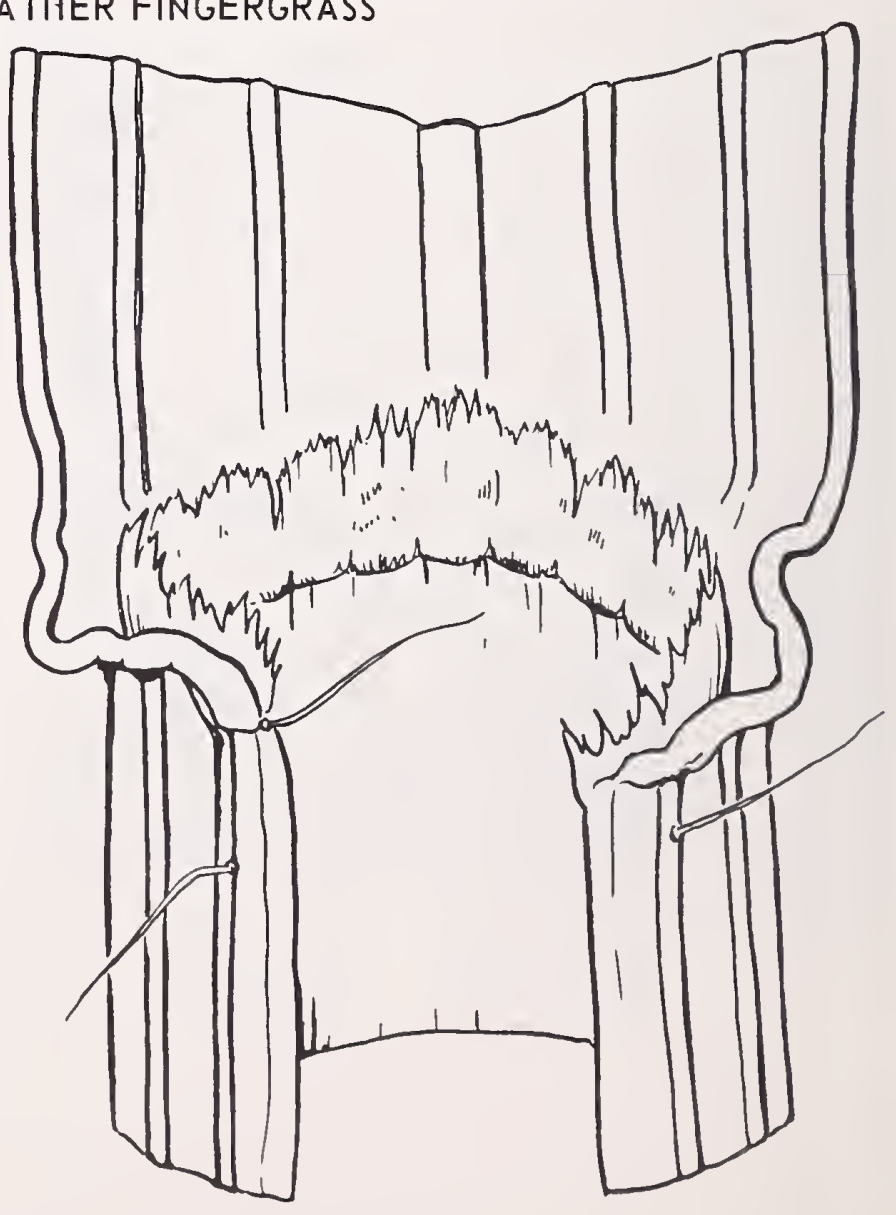


Decumbent, sodgrass. VERNATION Curled. BLADES - Flat, wide, short, usually glabrous; veins each side of midrib, 2 ; ribs not prominent; margin white, midrib prominent dorsally; 1-4 mm. wide, 2-6 $\mathrm{cm}$. long. AURICLE - None. LIGULE Hairy, medium, $1 \mathrm{~mm}$., with scattered long hairs. COLLAR - Usually glabrous, occasionally with long hairs. SHEATH Usually glabrous, hyaline margin, often with tufts of hair at the summit. NODES Glabrous. INTERNODES - Glabrous. ROOTS - Rhizomatous. CULM - Elliptical, branched, stoloniferous. OUTSTANDING CHARACTERS - Culms, stoloniferous, branched. Ligule hairy. Blade flat, short, wide. Growth decumbent except flower stalks.

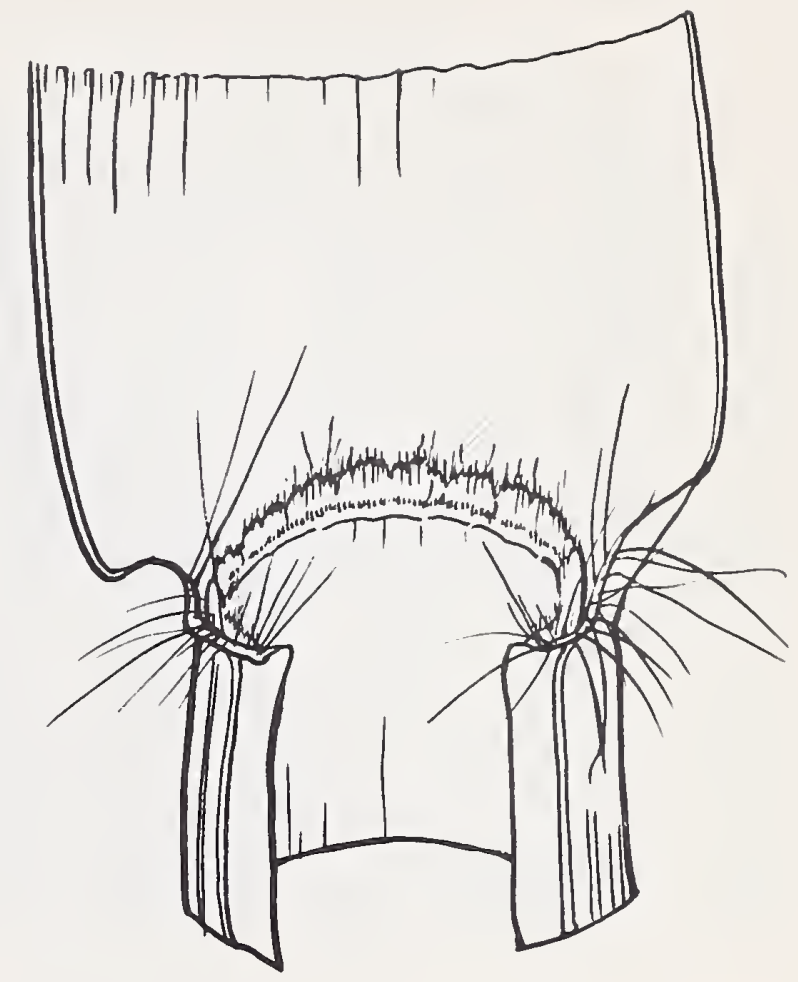

32. Distichlis stricta (Torr.) Rydb. DESERT SALTGRASS
Erect, semi-sodgrass. VERNATION Clasped. BLADES - Flat to rolled, erect, narrow, pointed; hairy ventrally, frequently with salt deposits; veins each side of midrib, 3; ribs prominent dorsally; margin toothed; midrib prominent ventrally; $2-3$ $\mathrm{mm}$. wide at base, 1-6 $\mathrm{cm}$. long. AURICLE - None. LIGULE - Hairy, small 1/2-1 mm. COLLAR - Occasionally hairy margin 1-2 mm. SHEATH - Smooth, round, paper margin. NODES - Glabrous. INTERNODES - Glabrous. ROOTS - Rhizomatous. CULM - Round, not branched. OUTSTANDING CHARACTERS - Rhizomatous. Blades usually rolled at tip, short. Lower culms and rhizomes conspicuously shiny.

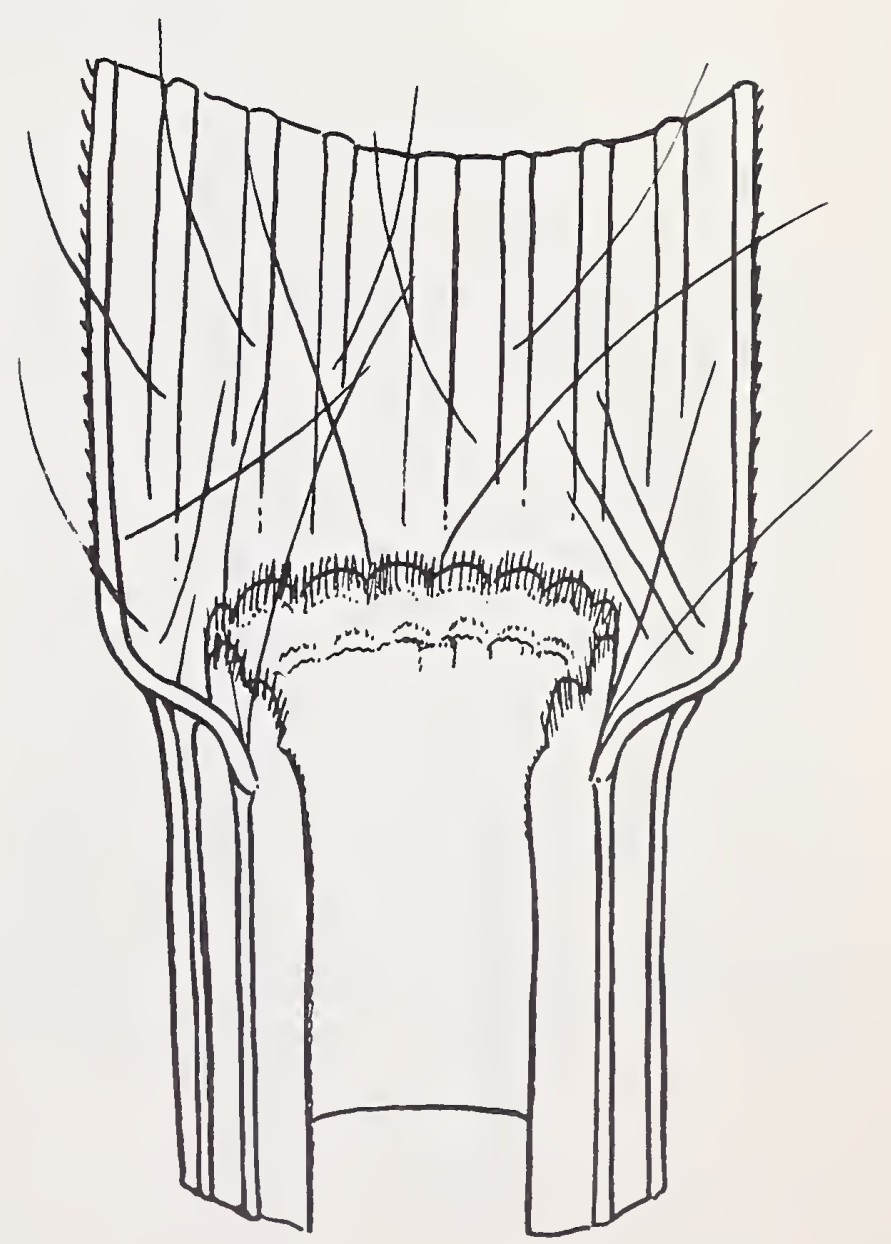


Erect, medium bunchgrass. VERNATION - Folded. BLADES - Rolled, narrow, long, hairy, ventrally, pilose, 3-5 mm.; ribs prominent dorsally; midrib not prominent; $1 \mathrm{~mm}$. wide, $15-30 \mathrm{~cm}$. long. AURICLE - None. LIGULE - Hairy, small $1 \mathrm{~mm}$., front pilose, 2-5 mm. COLLAR - Glabrous, divided. SHEATH - Glabrous, veined. NODES - Glabrous.INTERNODES - Pubescent to villous below node. ROOTS - Fibrous. CULM - Round, branched. OUTSTANDING CHARACTERS - Ligule hairy, front pilose, 2-5 $\mathrm{mm}$. Stems pubescent below node. Blades narrow, long rolled, hairy ventrally. Culms branched; heads silvery, densely pilose.

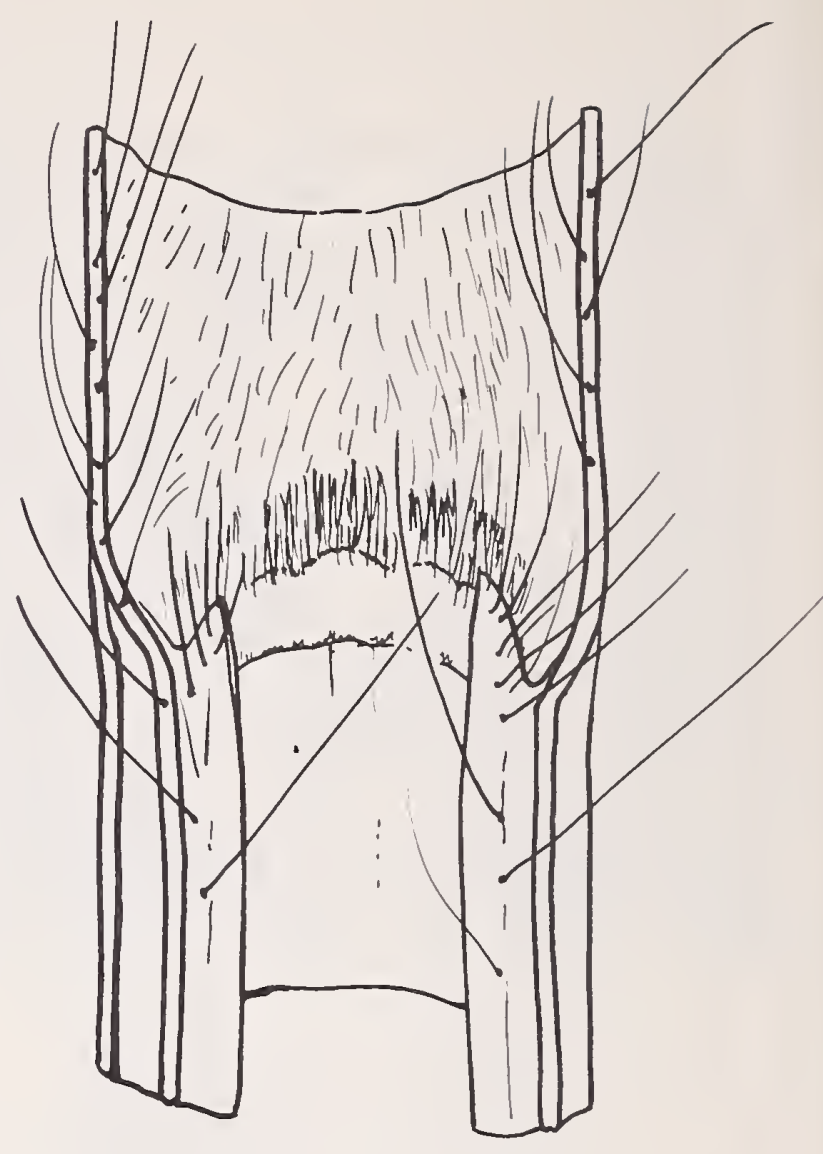

34. Eragrostis intermedia Hitchc. PLAINS LOVEGRASS

Erect, medium bunchgrass. VERNATION - Curled. BLADES - Flat, long, narrow, scattered hairs; veins each side of midrib, 3-4; ribs prominent ventrally and dorsally; margin smooth; midrib prominent dorsally; $2 \mathrm{~mm}$. wide, $10-30 \mathrm{~cm}$. long.AURICLE - None. LIGULE - Membranous, small 1/2 mm., truncate-ciliate, with long conspicuous hairs, 3-5 $\mathrm{mm}$. COLLAR - Hairy on margin. SHEATH Glabrous, veined. NODES - Glabrous.INTERNODES - Glabrous. ROOTS - Fibrous. CULM - Elliptical, not branched. OUTSTANDING CHARACTERS - Blades flat, long narrow. Conspicuous ribs on blade and sheath. Ligule membranous with long hairs. Collar bearded. Culm elliptical.

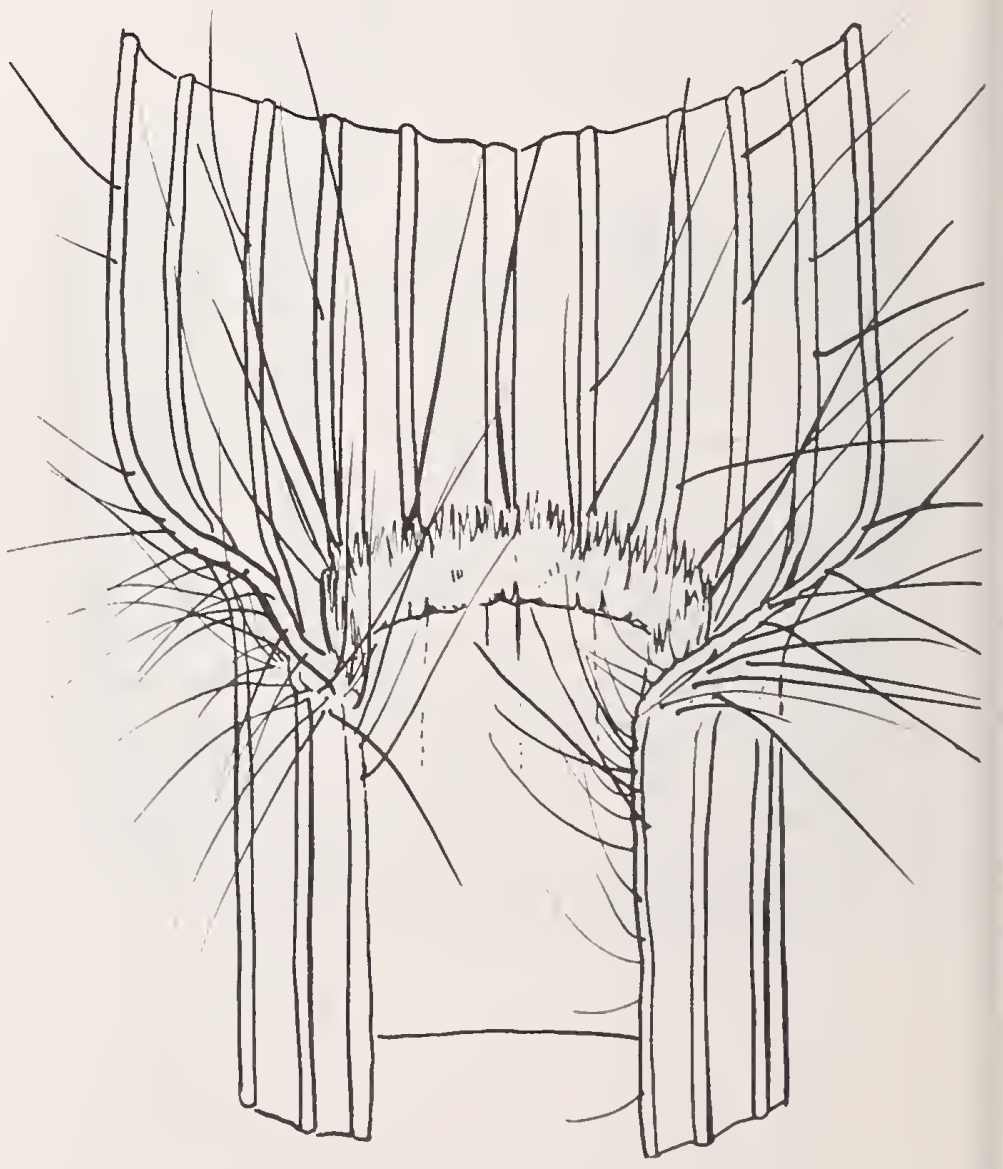


Erect, large bunchgrass. VERNATION - Curled. BLADES - Flat, drooping, wide, long, narrow, pointed; glabrous, soft; veins each side of midrib, 3; ribs prominent ventrally and dorsally; margin glabrous; midrib not prominent, 3-4 mm. wide, 12$15 \mathrm{~cm}$. long. AURICLE - None. LIGULE Hairy or membranous, truncate-ciliate, 1/2-1 mm. COLLAR - Hairy margin, 2-3 mm. SHEATH - Round, veined, frequently bearded at base. NODES - Occasionally pubescent. INTERNODES - Glabrous. ROOTS - Fibrous. CULM - Round, occasionally branched. OUTSTANDING CHARACTERS - Blades flat, long. Blade ribs prominent. Collar margin hairy, 2-3 $\mathrm{mm}$. Ligule truncate-ciliate, $1 / 2-1 \mathrm{~mm}$. Large leafy bunch. Culm round.

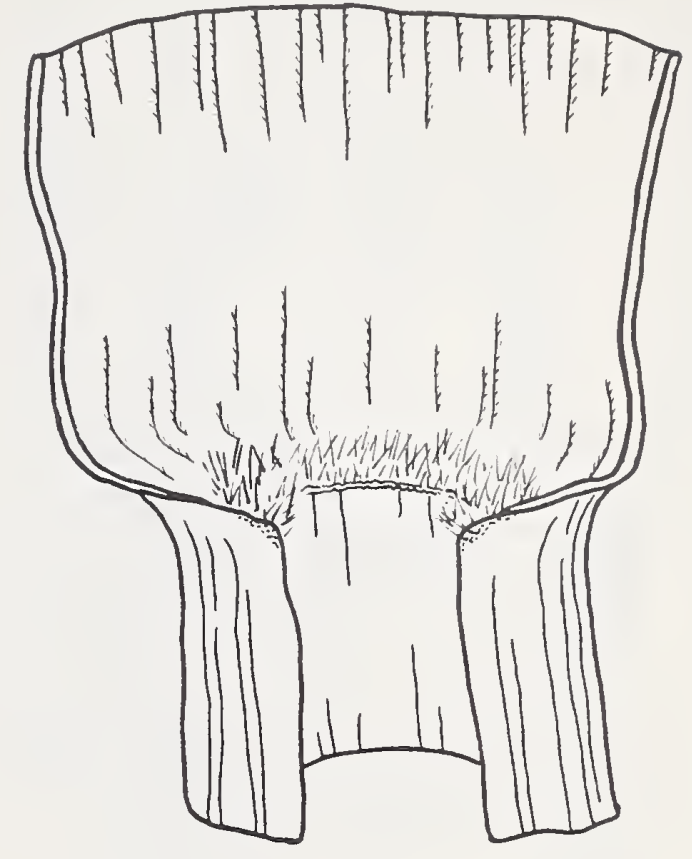

36. Festuca arizonica Vasey. ARIZONA FESCUE

Erect, large bunchgrass. VERNATION - Folded. BLADES - Rolled, drooping, very small; glabrous; ribs indistinct; margin toothed; midrib indistinct; $1 / 4-1 / 2 \mathrm{~mm}$. wide, 10-30 cm. long. AURICLE - None. LIGULE - Membranous; very small, 1/4$1 / 2 \mathrm{~mm}$; acute-entire. COLLAR - Indistinct. SHEATH - Elliptical, pinkish above roots. NODES - Glabrous. INTERNODES Glabrous. ROOTS - Fibrous. CULM Round, not branched. OUTSTANDING CHARACTERS - Long drooping, rolled, thread-like blades. Large bunches. Ligule very small and difficult to observe.

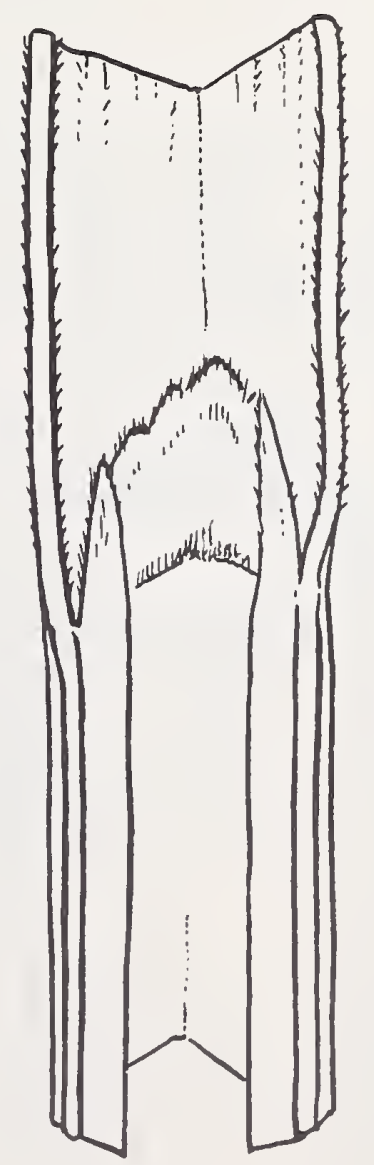


Erect, small bunchgrass. VERNATION - Folded. BLADES - Flat, wide; hairy occasionally ventrally; veins each side of midrib, 3-4; ribs not prominent; margin glandular at base, white; midrib prominent ventrally; 6-8 mm. wide, 6-15 cm. long. AURICLE - None. LIGULE - Hairy, lower half membranous, overall length $1 \mathrm{~mm}$. COLLAR - Hairy margin, glandular, 2-4 mm. SHEATH - Flat, glabrous, veined. NODES - Glabrous. INTERNODES - Glabrous. ROOTS - Fibrous. CULM - Flat, not branched. OUTSTANDING CHARACTERS - Blades flat, wide, occasionally folded at base. Culm flat. Blade margin and collar with glandular hairs. Ligule hairy, small. Midrib prominent ventrally. Awns long, hairy, tangled mass.

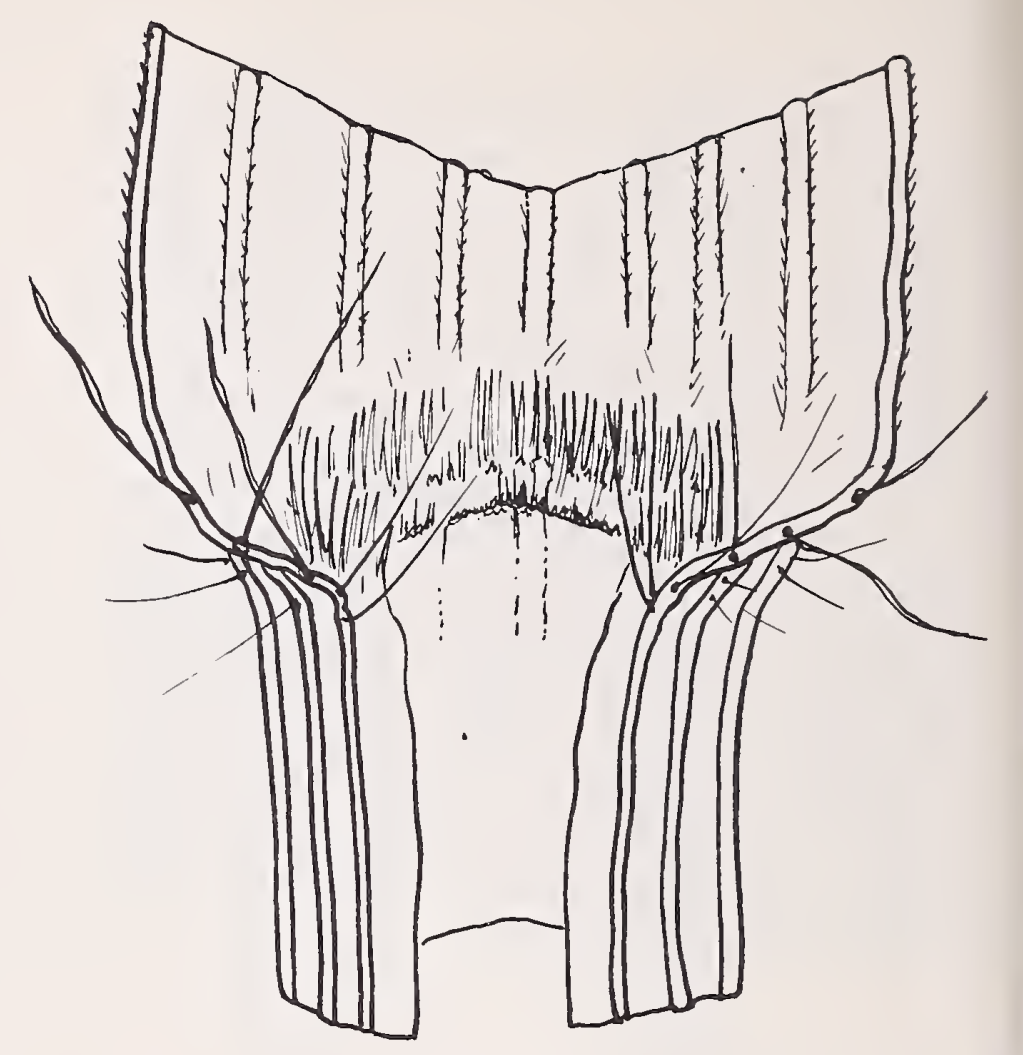

38. Hilaria belangeri (Steud.) Nash. CURLYMESQUITE

Open sodgrass. VERNATION - Curled. BLADES - Flat, curled, narrow, pointed, long; hairy, glandular; veins on each side of midrib, 2; ribs not prominent ventrally; $2 \mathrm{~mm}$. wide, 5-20 cm. long. AURICLE None. LIGULE - Membranous, large $2 \mathrm{~mm}$.; truncate-lacerate. COLLAR - Glabrous. SHEATH - Glabrous, paper margin. NODES - Hairy。INTERNODES - Glabrous。ROOTS - Fibrous. CULM - Round, lower nodes grow roots and culms. OUTSTANDING CHARACTERS - Culms root at lower nodes. Nodes hairy. Blades with glandular hairs, 2-4 $\mathrm{mm}$. Ligule $2 \mathrm{~mm}$. truncatelacerate.

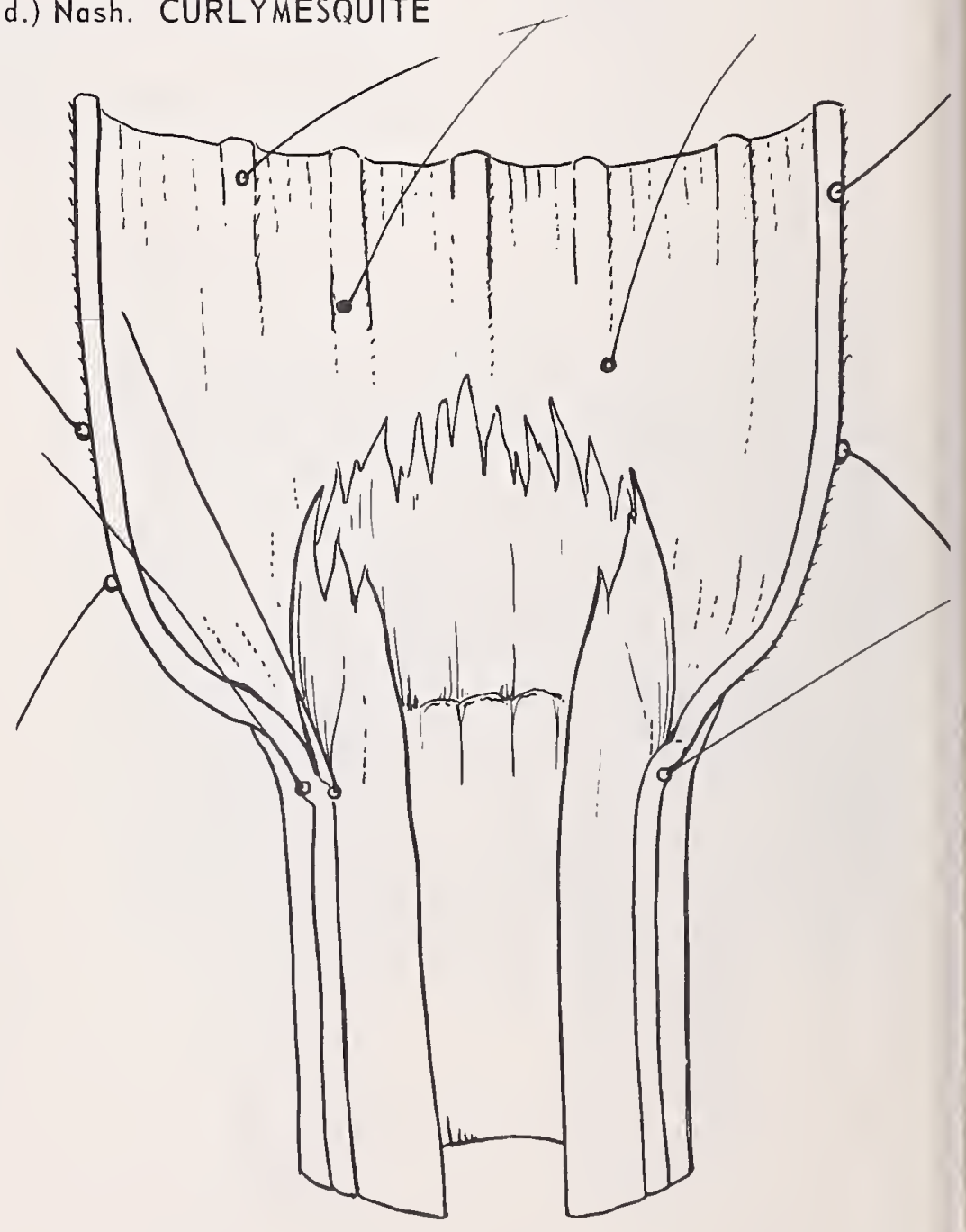


Erect, large bunchgrass. VERNATION - Curled. BLADES - Flat base, upper 2/3 often rolled, pointed; rough ventral and dorsal; veins each side of midrib, 3-4; ribs prominent dorsally; margin toothed; midrib not prominent; $3-5 \mathrm{~mm}$. wide at base, 4-12 cm. long. AURICLE - None. LIGULE - Membranous, 1-3 mm., truncate-lacerate. COLLAR - Usually glabrous. SHEATH - Elliptical, veined. NODES Pubescent to villous. INTERNODES - Often pubescent below node. ROOTS - Rhizomatous. CULM - Round, not branched. OUTSTANDING CHARACTERS - Blades flat base, upper rolled. Rhizomatous. Nodes pubescent. Ligule 1-2 mm., truncatelacerate. Culm base frequently semidecumbent. Rachis zigzag.

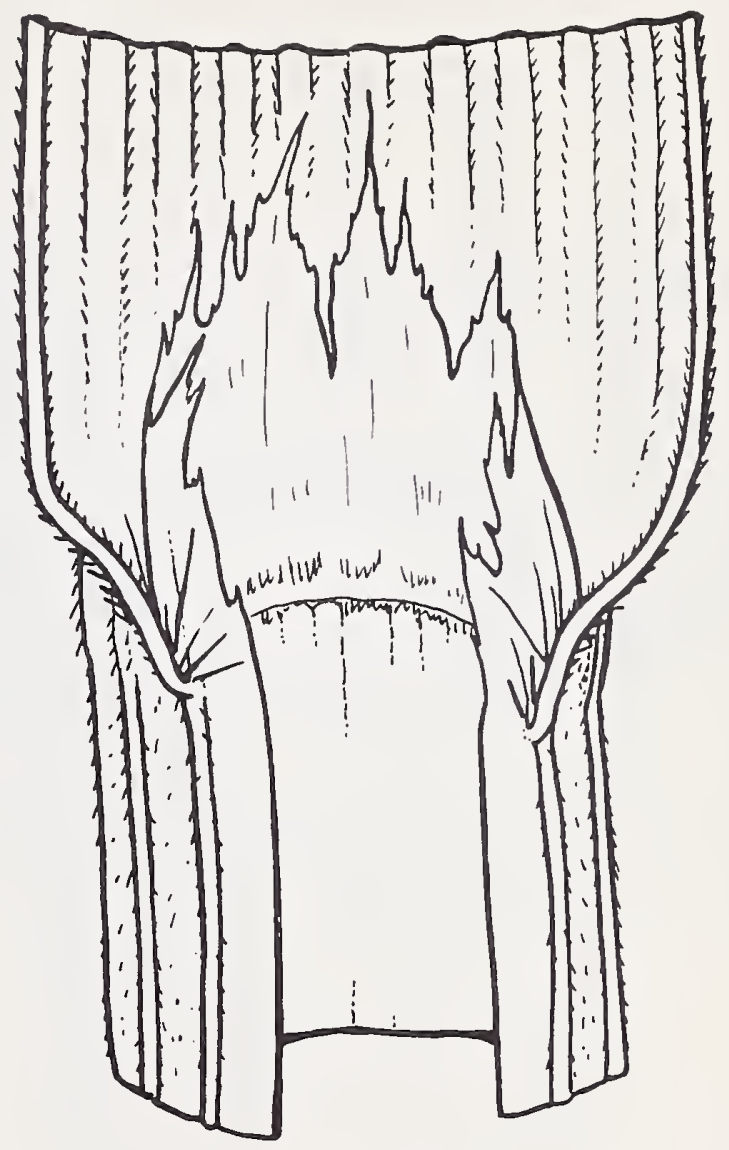

40. Hilaria mutica (Buckl.) Benth. TOBOSA

Big bunchgrass, semi-erect. VERNATION - Curled. BLADES - Flat to rolled; occasionally hairy ventrally; veins each side of midrib, 2-3; ribs prominent; midrib prominent ventrally; $2-4 \mathrm{~mm}$. wide, 5-10 cm. long. AURICLE - None. LIGULE Membranous, truncate-ciliate, 1-2 mm. COLLAR - Hairy margin. SHEATH - Glabrous, veined. NODES - Glabrous to pubescent. INTERNODES - Glabrous. ROOTS Rhizomatous. CULM - Round, branched. OUTSTANDING CHARACTERS - Blade usually rolled. Growth semi-decumbent, large bunchgrass. Rhizomatous. Culms branch. Ligule truncate-ciliate.

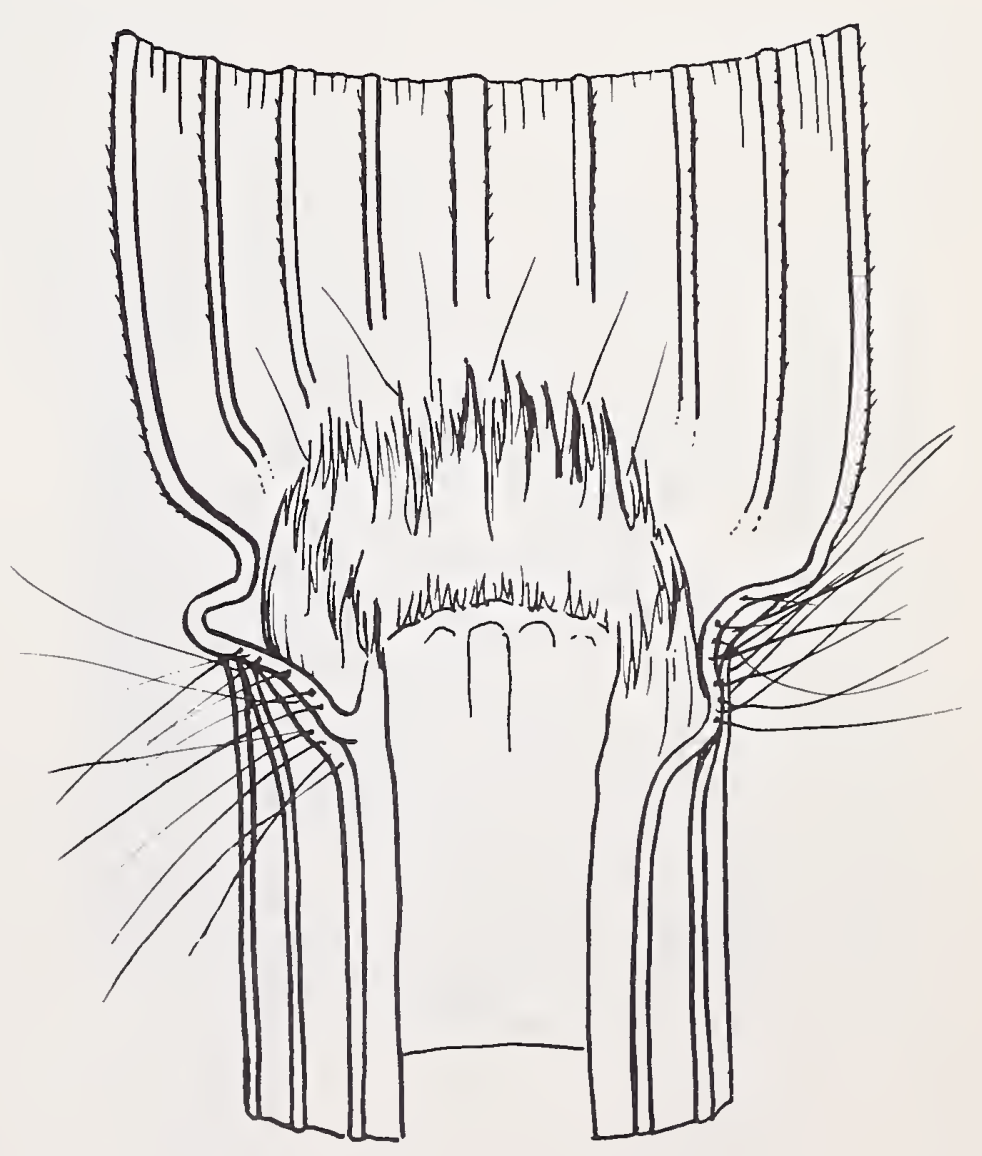


Erect, large bunchgrass. VERNATION - Curled. BLADES - Flat at base, upper part rolled, erect, narrow, pointed; smooth, stiff; veins each side midrib 2-4; ribs prominent ventrally, 6-8; margin toothed; midrib prominent dorsally; width 1-2 $\mathrm{mm}$. length 4-10 cm. AURICLE - None. LIGULE - Hairy to woolly, small, $1 \mathrm{~mm}$. COLLAR Woolly. SHEATH - Round, margin woolly. NODE - Pubescent to woolly. INTERNODE - Woolly. ROOTS - Hard. scaly, rhizomatous. CULMi - Round, branched. OUTSTANDING CHARACTERS - New growth sprouts at nodes. Ligule, nodes, collar, sheath and culms woolly.

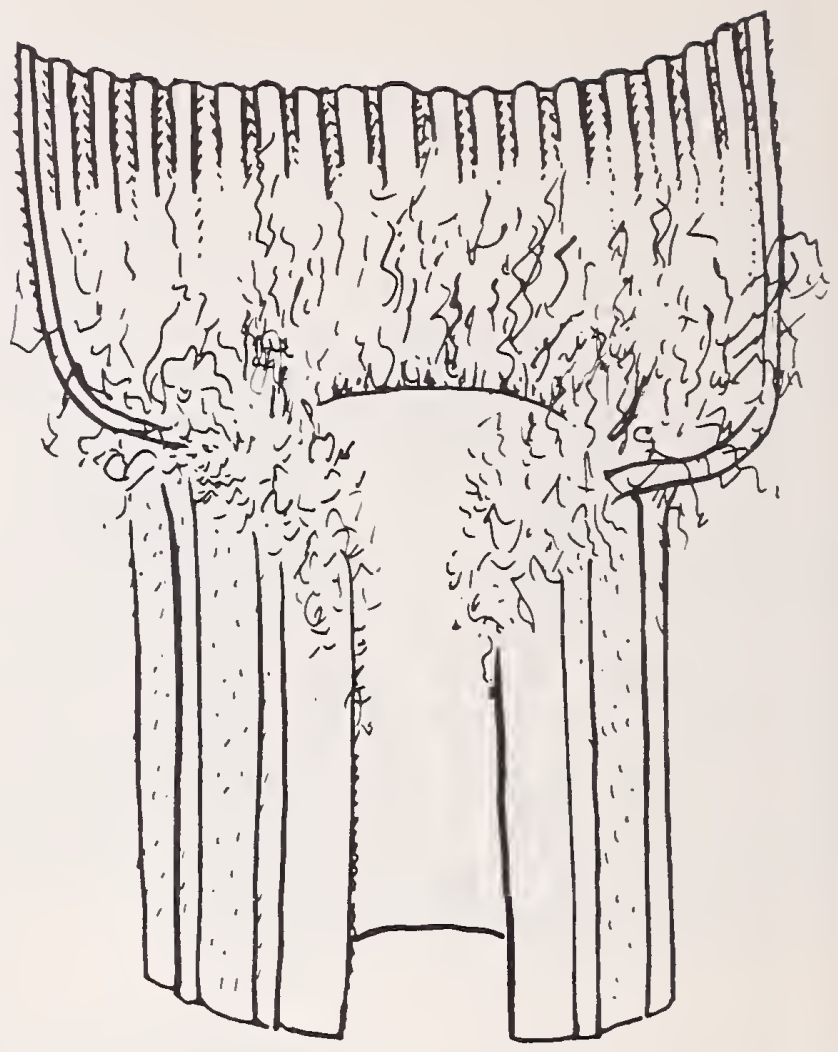

42. Koeleria cristata (L.) Pers. JUNEGRASS

Erect, small bunchgrass. VERNATION - Folded. BLADES - Flat, drooping, narrow, pointed; rough ventrally, canescent dorsally, soft; veins each side of midrib usually 2; ribs prominent ventrally; margin toothed; width $2 \mathrm{~mm}$., length $5-15 \mathrm{~cm}$. AURICLE - None. LIGULE - Membranous, small 1/4-1/2 mm. truncate-ciliate. COLLAR - Glabrous to canescent. SHEATH Usually canescent, round. NODES - Usually glabrous. INTERNODES - Usually canescent above node. ROOTS - Fibrous. CULM - Round, not branched. OUTSTANDING CHARACTERS - Blade flat, conspicuously ribbed ventrally, soft. Internode canescent near node. Ligule membranous, truncate-ciliate $1 / 2 \mathrm{~mm}$.

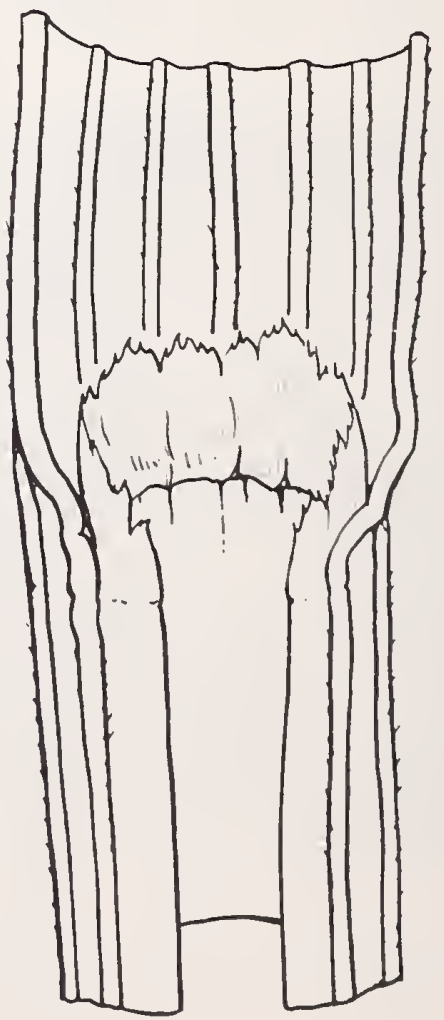


Erect, medium bunchgrass. VERNATION - Folded (reported in Colorado as curled). BLADES - Flat, wide, long, drooping; occasional hairs dorsally 2-5 mm., on lower one-third; veins on each side of midrib 2-3; ribs ventral and dorsal; margin toothed; midrib prominent ventrally; width 4-5 mm. length $15-25 \mathrm{~cm}$. AURICLE - None. LIGULE - Hairy, small 1/2 mm., margin hairs 3-5 mm. COLLAR - Hairy margin, 3-5 mm. SHEATH - Glabrous, veined, papery margin. NODE - Glabrous. INTERNODE - Glabrous. ROOTS - Fibrous. CULM - Flat, not branched. OUTSTANDING CHARACTERS - Collar margin hairy. Culms flat. Ligule hairy, small $1 / 2 \mathrm{~mm}$. occasional hairs $3-5 \mathrm{~mm}$. Blades flat, wide, long drooping. Sheath veined, papery margin.

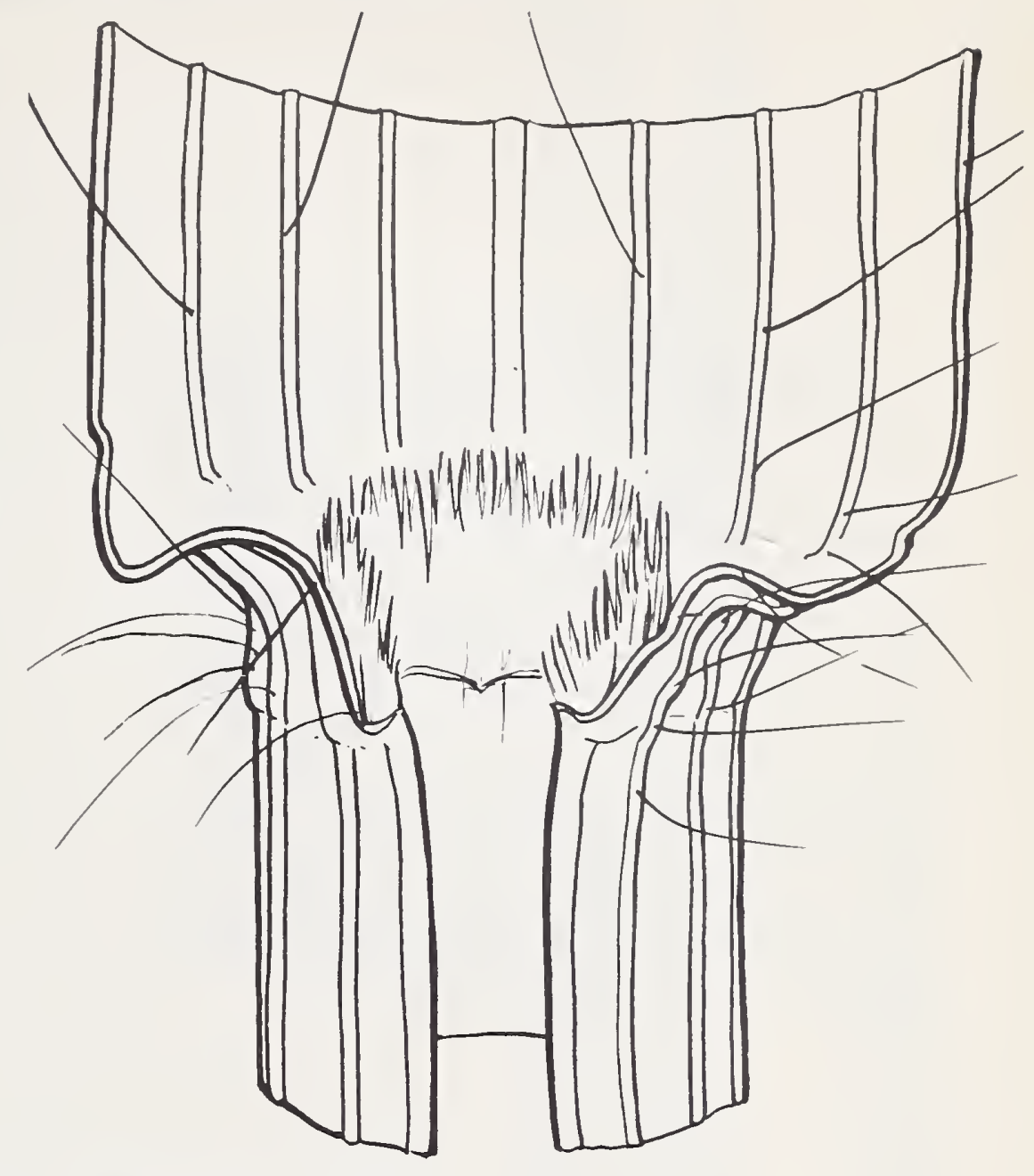

44. Lolum perenme L. PERENNIAL RYEGRASS

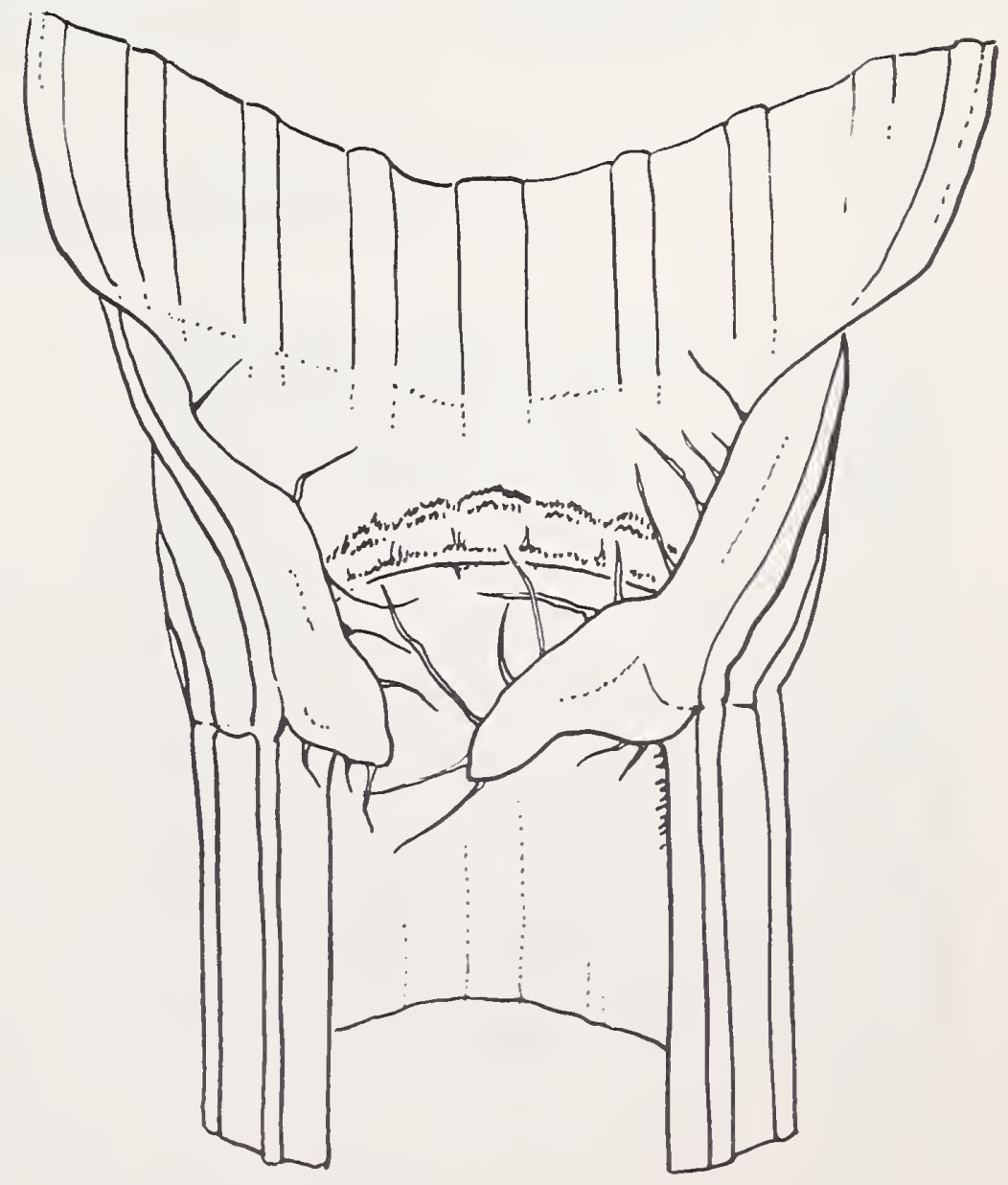


Erect, small bunchgrass. VERNATION - Folded. BLADES - Usually folded, drooping, glabrous; veins each side of midrib 2; margin white, toothed; ribs indistinct; midrib prominent dorsally; width $1 \mathrm{~mm}$., length 3-10 cm. AURICLE - None. LIGU LE - Membranous, large, 2-5 mm. acuteentire. COLLAR - Glabrous. SHEATH Flat, hyaline white margin. NODE - Short canescent. INTERNODE - Glabrous to short canescent near nodes. ROOTS Fibrous. CULM - Flat, branched. OUTSTANDING CHARACTERS - Blades and sheath conspicuous white margin, flattened at base. Ligule large acute. Culms branch at nodes. Blade usually folded.

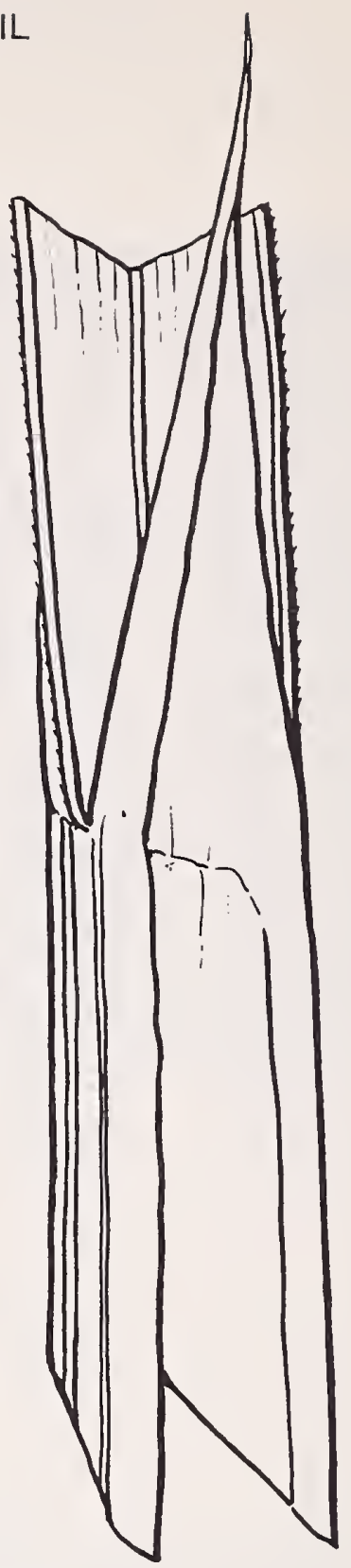

46. Muhlenbergia curtifolia Scribn. UTAH MUHLY

Erect, medium sized bunchgrass. VERNATION - Clasped. BLADE - Rolled, semi-erect, pointed; rough, stiff, pubescent ventrally; veins each side of midrib 3-4; ribs prominent dorsally; margin toothed; midrib prominent ventrally; width $1 \mathrm{~mm}$., length $1-21 / 2 \mathrm{~cm}$. AURICLE None. LIGULE - Membranous, small 1-2 mm., acute-lacerate. COLLAR - Glabrous. SHEATH - Glabrous, veined, round. NODE - Glabrous. INTERNODE - Pubescent to glabrous. ROOTS - Fibrous. CULM Round, not branched. OUTSTANDING CHARACTERS - Ligule membranous, 1-2 mm., acute-lacerate. Blades rolled, long and narrow. Blade veins 3-4. Plant glabrous.

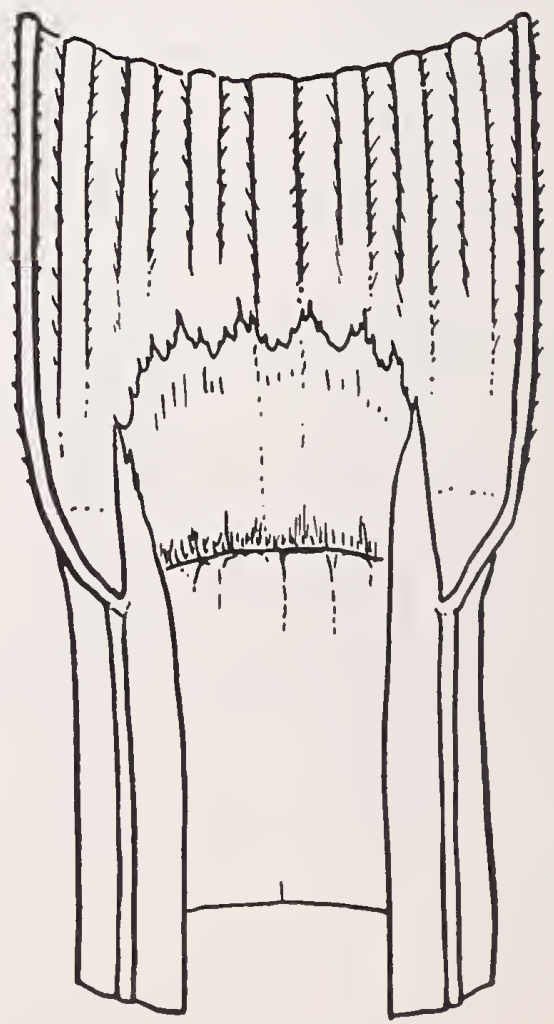


Decumbent, large bunchgrass. VERNATION - Curled. BLADES - Flat, narrow, short, pointed; rough dorsally; veins each side of midrib 2; ribs not prominent; margin toothed, midrib not prominent; width $1 \mathrm{~mm}$., length 3-8 cm. AURICLE - None. LIGULE - Membranous, 1-2 mm., hyaline, truncate-lacerate. COLLAR - Glabrous. SHEATH - Open, glabrous, veined. NODE Glabrous. INTERNODE - Glabrous. ROOTS - Fibrous. CULM - Elliptical to round, branched. OUTSTANDING CHARACTERS Ligule conspicuous, truncate-lacerate, 1-2 $\mathrm{mm}$. Blade short, flat. Branches at nodes. Usually found clambering over shrubs. Culms small, long and semi-woody, persistently green. Florets purple awned.

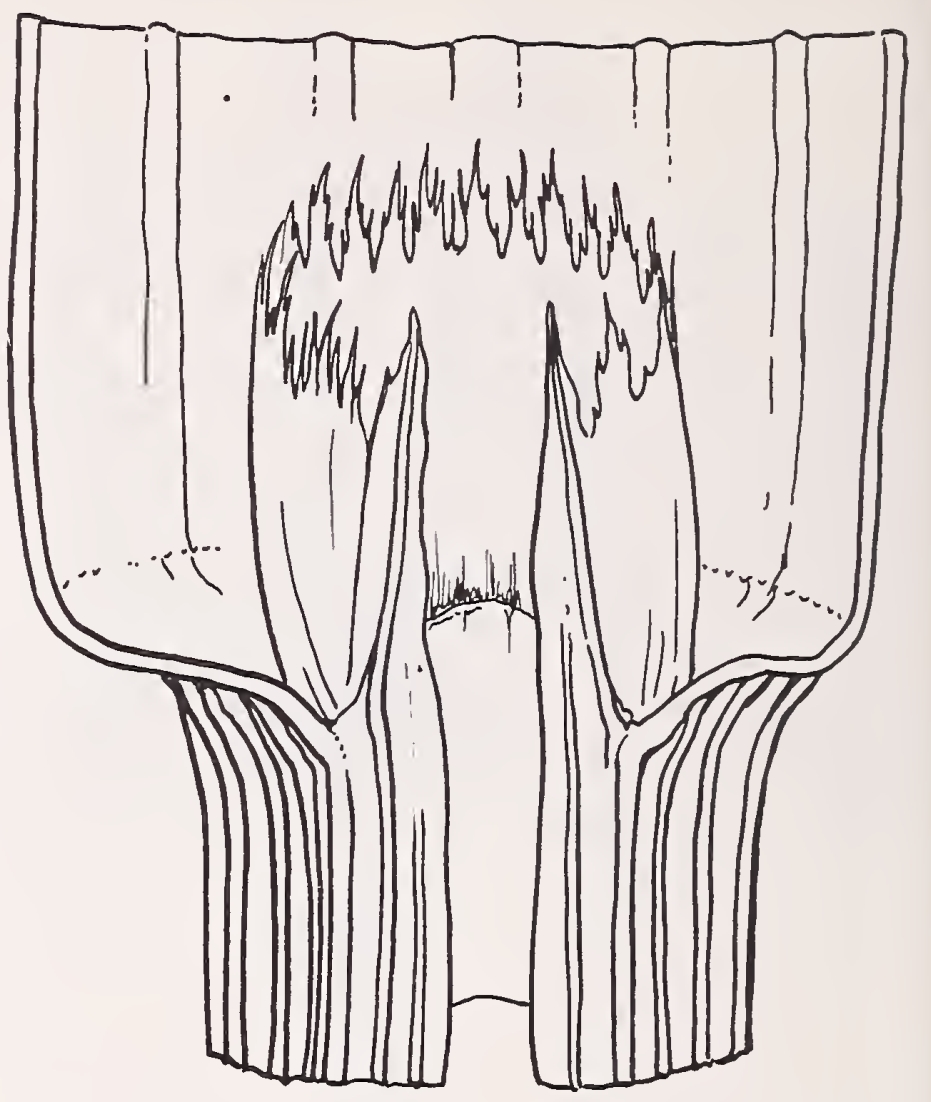

50. Nuhlenbergia pungens Thurb. SANDHILL MUIHLY

Semi-erect, big bunchgrass. VERNATION - Clasped. BLADES - Rolled, very sharp pointed (pungent); rough ventrally, stiff; ribs indistinct; width $1 \mathrm{~mm}$., length 3-5 cm. AURICLE - None. LIGULE Membranous, small, $1 / 2 \mathrm{~mm}$; truncateciliate. COLLAR - Glabrous. SHEATH Open, glabrous, round, papery margined. NODE - Canescent. INTERNODE - Canescent. ROOTS - Rhizomatous. PANICLE Reddish-brown. CULM - Round. OUTSTANDING CHARACTERS - Node - internode canescent. Blades rolled pungent. Rhizomatous. Prefers sandy soil. Panicle open, reddish.

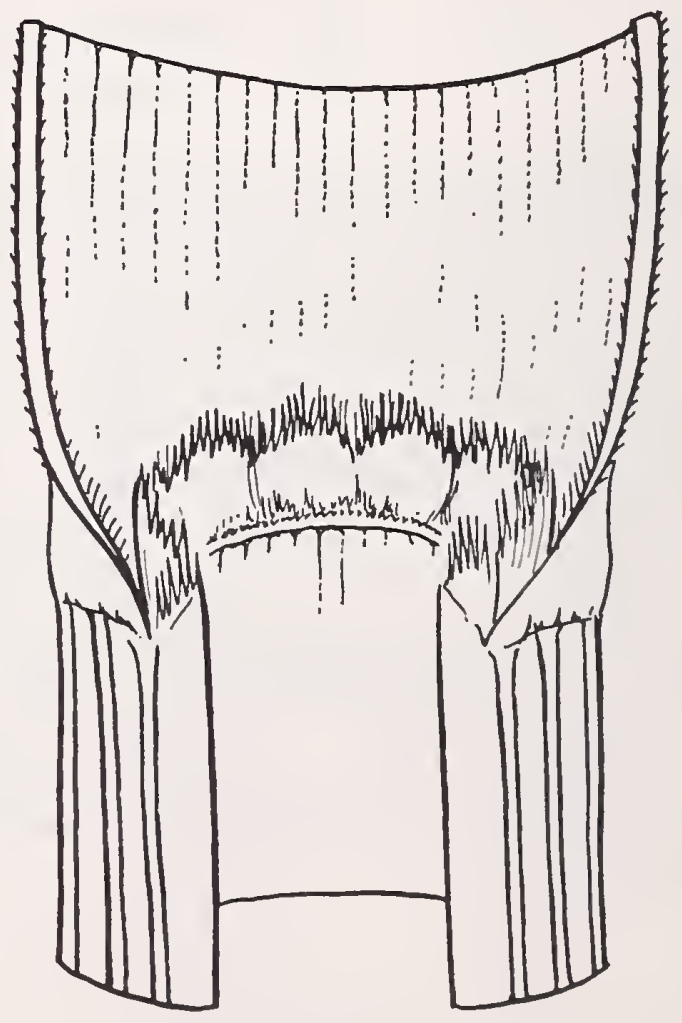


Erect to semi-erect, branched, forming a mat. VERNATION - Folded. BLADES Rolled, erect, narrow, pointed; glabrous, rough ventrally, stiff; margin toothed; width $1 / 2 \mathrm{~mm}$., length $1-5 \mathrm{~cm}$. AURICLE None. LIGULE - Membranous, small 1/2-1 mm., truncate-toothed. COLLAR - Glabrous. SHEATH - Glabrous, white hyaline margin, elliptical. NODE - Glabrous. INTERNODE - Glabrous. ROOTS - Fibrous, short rhizomes. CULM - Elliptical, branched. OUTSTANDING CHARACTERS Growth a dense mat. Sheath margin white. Blades short, rolled, erect.

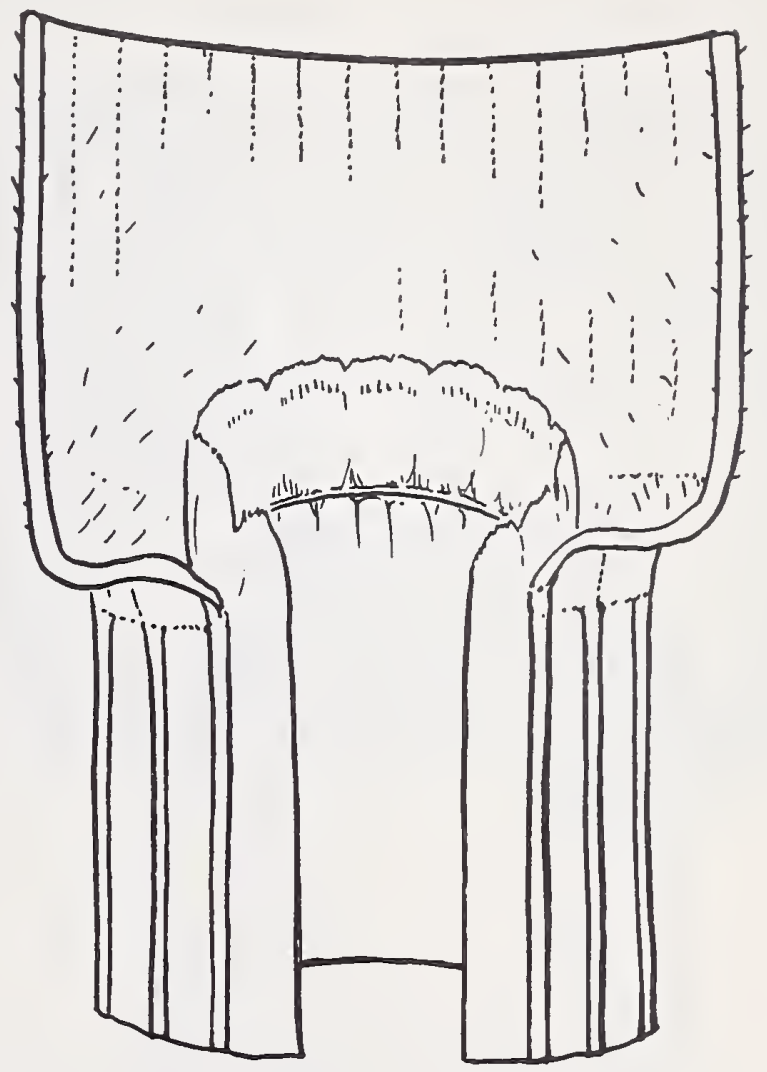

52. Muhlenbergia rigens (Benth.) Hitchc. DEERGRASS

Erect, large bunchgrass. VERNATION - Clasped. BLADES - Flat to rolled, rough, pointed, veins each side of midrib 2; ribs prominent ventrally and dorsally; margin toothed; width 1-2 mm., length $10-20 \mathrm{~cm}$. AURICLE - None. LIGULE - Membranous, 2-4 mm., obtuse-lacerate. COLLAR Glabrous. SHEATH - White margin, round, glabrous. NODE - Glabrous. INTERNODE Glabrous. ROOTS - Fibrous. CULM Round, not branched. OUTSTANDING CHARACTERS - Large bunchgrass. Blades long, narrow, rolled to flat. Ligule membranous, large, acute-lacerate, 2-4 $\mathrm{mm}$.

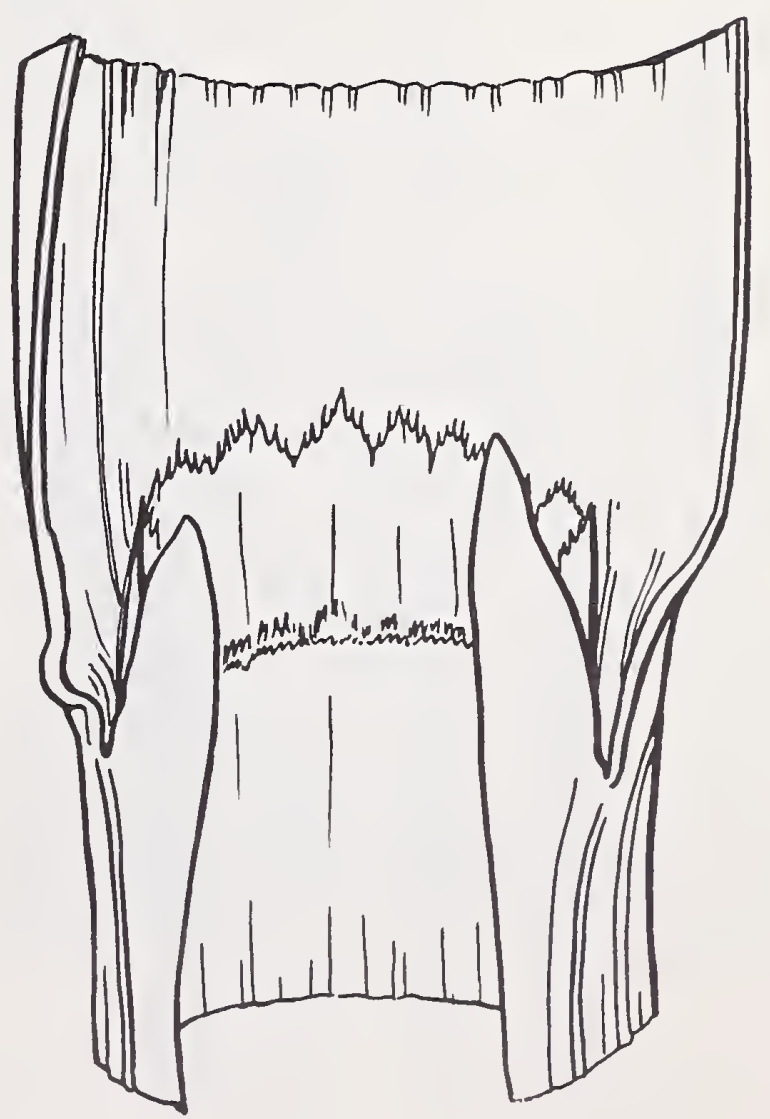


Semi-erect, dense mat, frequently open in center, or ring-like. VERNATION Folded. BLADES - Small, folded, erect, narrow, pointed; glabrous; midrib not prominent; width $1 / 2 \mathrm{~mm}$., length $1-2 \mathrm{~cm}$. AURICLE - None. LIGULE - Membranous, medium 2-3 mm., acute-entire. COLLAR Glabrous. SHEATH - Glabrous, veined, round. NODE - Canescent. INTERNODE Canescent. ROOTS - Short rhizomes. CULM - Flat, not branched, very small. OUTSTANDING CHARACTERS - Growth often ring-like, forming a dense mat. Very small blades and stems, flat. Ligule prominent.

54. Oryzopsis hymenoides (Roem. \& Schult.) Ricker. INDIAN RICEGRASS

Erect, small bunchgrass. VERNATION - Clasped or curled. BLADES - Rolled, drooping, narrow, pointed; rough dorsally; midrib prominent dorsally; width $1 / 2 \mathrm{~mm}$., length 5-30 cm. AURICLE - None. LIGULE - Miembranous, hyaline 2-3 mm., acuteentire. COLLAR - Glabrous to pubescent. SHEATH - Hairy margin, $1 \mathrm{~mm}$. NODE Glabrous. INTERNODE - Glabrous to pubescent. ROOTS - Fibrous. CULM - Round, not branched. OUTSTANDING CHARACTERS - Sheath usually hairy margin. Ligule hyaline, acute-entire. Blade rolled, narrow, long. 
Erect, small bunchgrass. VERNATION - Folded. BLADES - Folded to flat, drooping, narrow, pointed; occasional glandular hairs near ligule; veins each side of midrib 3-4; rib prominent ventrally and dorsally, margin white, midrib prominent dorsally, width $2 \mathrm{~mm}$., length $10-20 \mathrm{~cm}$. AURICLE - None. LIGULE - Membranous, medium $1 \mathrm{~mm}$., truncate-ciliate. COLLAR - Glabrous, divided. SHEATH - Elliptical, occasional glandular hairs near collar. NODE - Glabrous。INTERNODE - Glabrous。 ROOTS - Corm, rhizomes. CULM - Flat, not branched. OUTSTANDING CHARACTERS - Bulbs prominent. Occasional glandular hairs on blade and sheath.

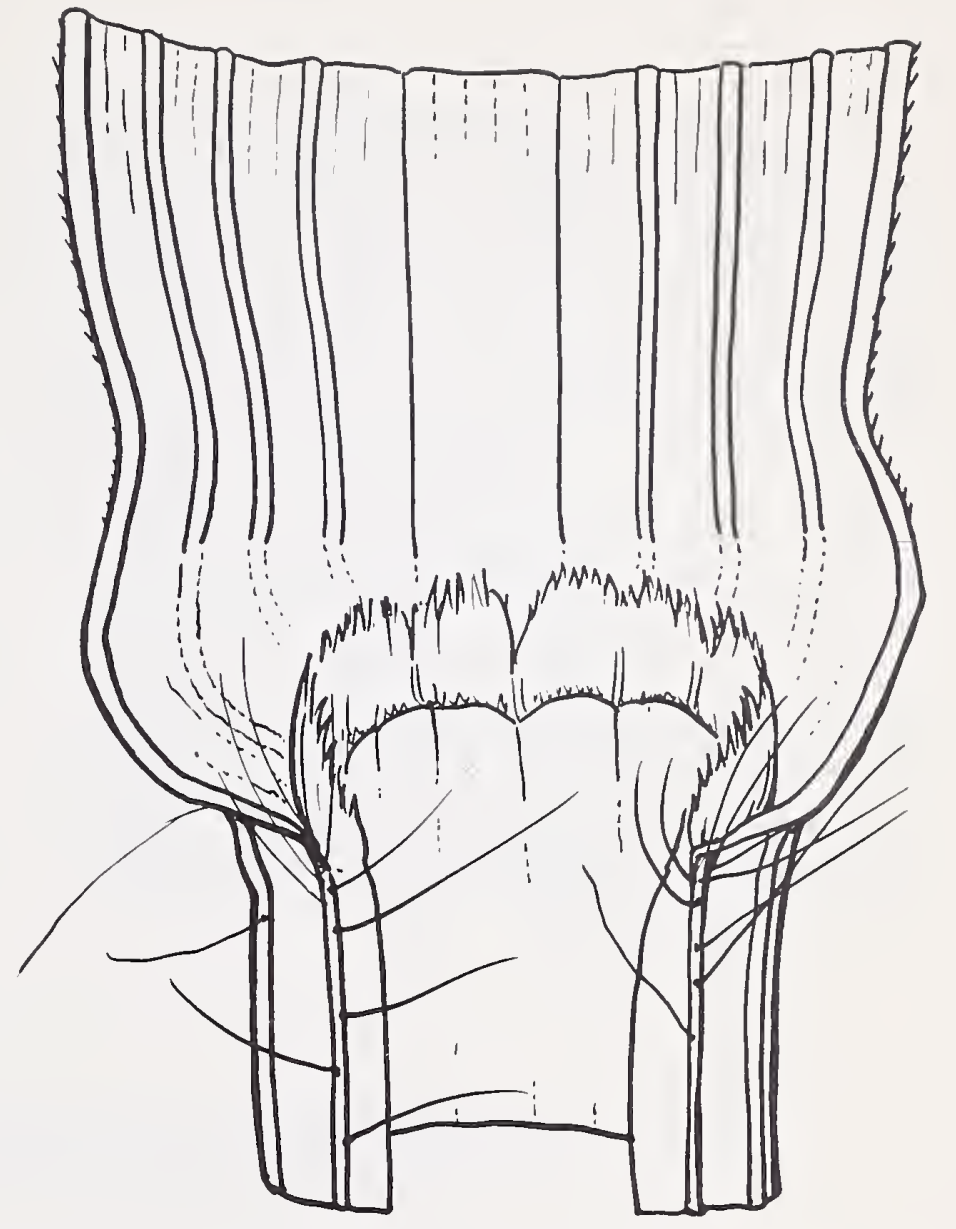

56. Panicum obtusum H.B.K. VINE-MESOUITE

Erect, almost a sod, strongly stoloniferous. VERNATION - Curled. BLADES Flat, wide, long; glandular hairs ventrally; veins each side of midrib 4; ribs prominent; margin toothed; midrib prominent dorsally; width 4-6 mm., length 10-20 cm. AURICLE - None. LIGULE - Membranous, with occasional hairs 1-3 $\mathrm{mm}$., obtusenotched. COLLAR - Margin long-hairy. SHEATH - Glandular hairs at base of plant. NODE - Glabrous (Stolons hairy). INTERNODE - Glabrous. ROOTS - Stoloniferous. CULM - Elliptical, not branched. OUTSTANDING CHARACTERS - Blades flat, wide, long, stiff, narrow, pointed. Ligule membranous also long hairs $3-5 \mathrm{~mm}$. Strongly stoloniferous with stolons up to 10 feet long. Sheath with glandular hairs at base of plant.

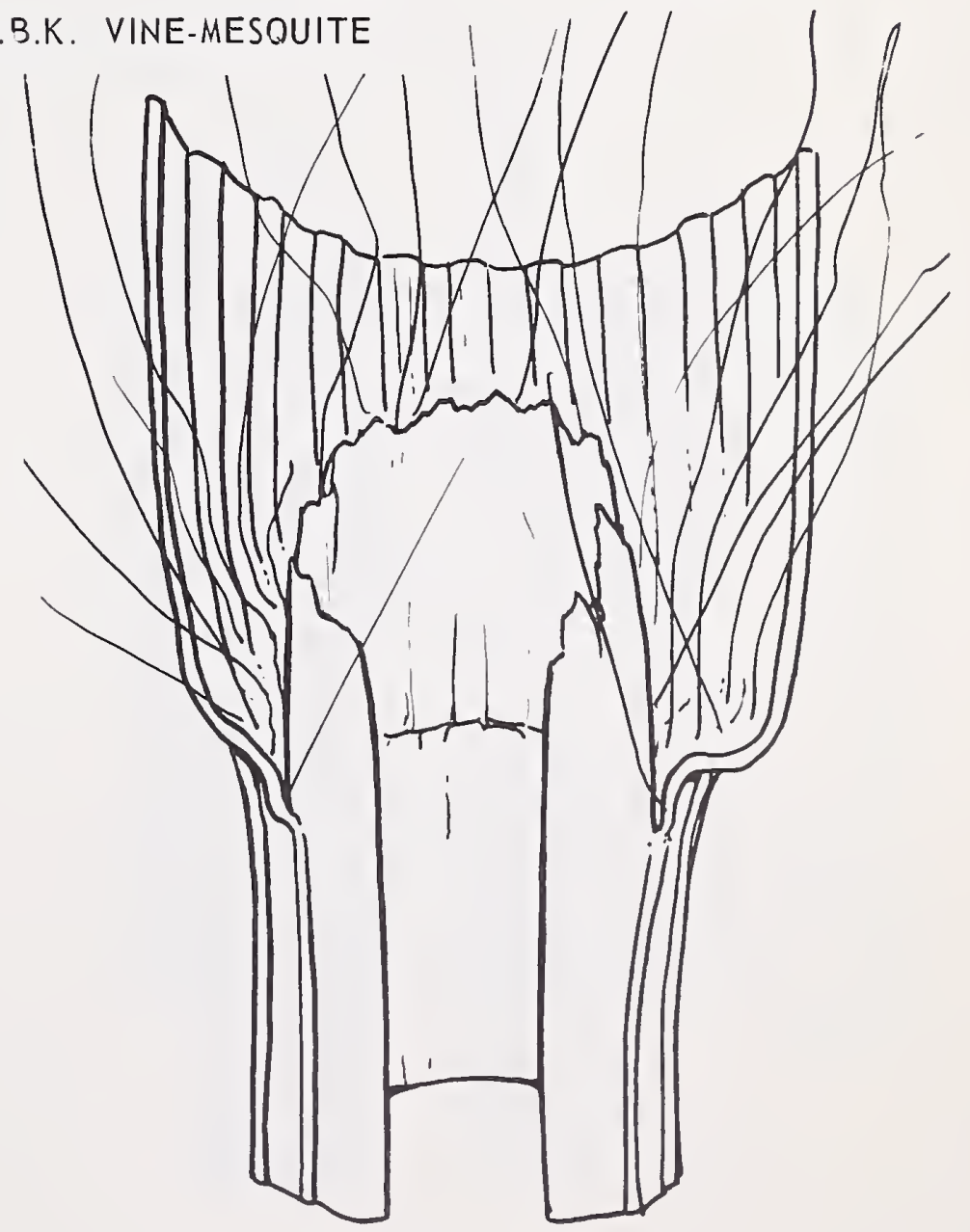


Erect, large bunchgrass. VERNATION - Curled. BLADES - Flat, drooping, narrow, pointed; rough ventrally, soft; veins each side of midrib $3-4$; ribs prominent dorsally; margin toothed; midrib not prominent; width 3-4 mm., length 15-30 $\mathrm{cm}$. AURIClE - None. LIGULE - Membranous, 2-4 mm., bearded, truncateciliate. COLLAR - Glabrous. SHEATH Glabrous, round, paper-like margin. NODE - Glabrous. INTERNODE - Glabrous. ROOTS - Fibrous. CULM - Round, branched at nodes. OUTSTANDING CHARACTERS - Culms branch at nodes. Ligule membranous 2-4 mm., truncate-ciliate. Blades flat, drooping, narrow, pointed, 15-30 cm. long.

\section{SS}

58. Poa bigelovii Vasey \& Scribn. BIGELOW BLUEGRASS

Erect, small annual bunchgrass. VERNATION - Folded. BLADES - Flat, wide, drooping, narrow, pointed, toothed; glabrous; veins each side of midrib 2; midrib not prominent; margin toothed; ribs not prominent; width 2-4 mm., length $4-12 \mathrm{~cm}$., the tip boat-shaped. AURICLE - None. LIGULE - Membranous, hyaline 2-4 mm., acute-lacerate. COLLAR - Glabrous, divided. SHEATH - Glabrous, margin hyaline. NODE - Glabrous. INTERNODE Glabrous. ROOTS - Fibrous, annual. CULN: - Elliptical, not branched. OUTSTANDING CHARACTERS - Annual. Plant glabrous. Ligule hyaline 2-4 mm., acute-lacerate. Blade flat, wide, drooping, long, narrow, pointed.

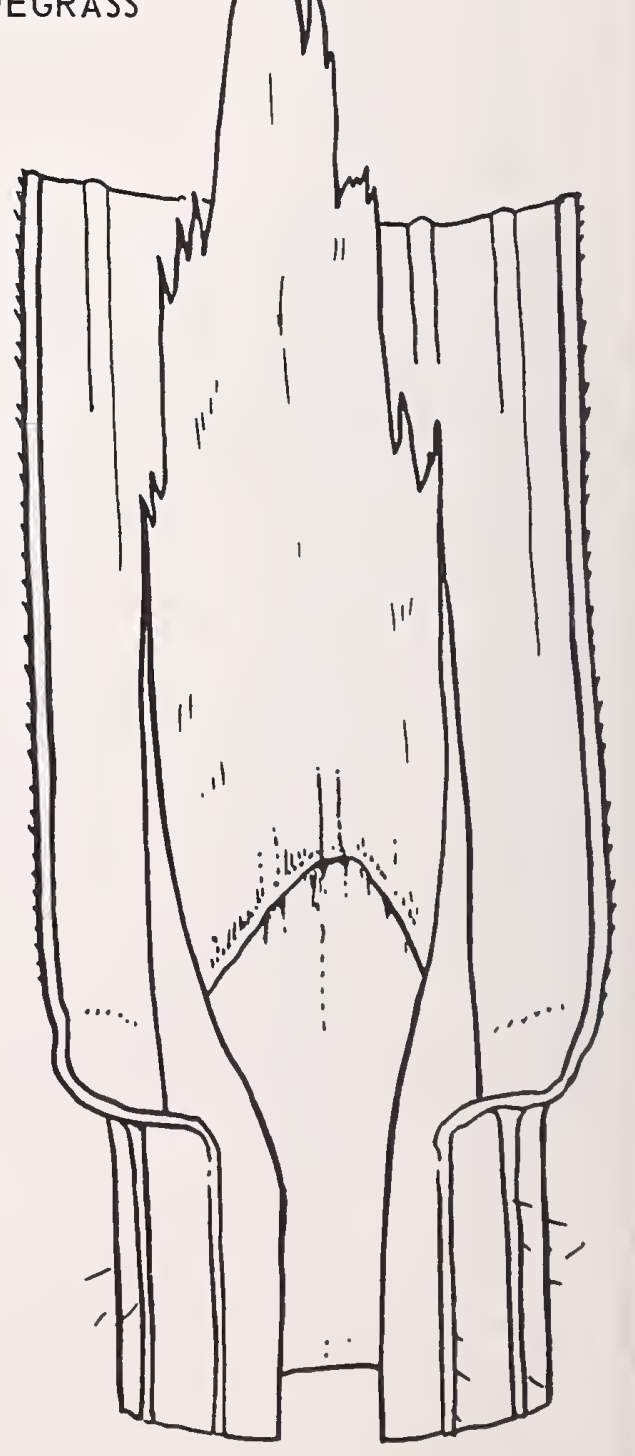


Erect, small bunchgrass. VERNATION - Folded. BLADES - Folded, erect, glabrous, stiff; ribs dorsal; margin glabrous; width 1/2-1 mm., length 10-20 $\mathrm{cm}$., the tip boat-shaped. AURICLE - None LIGULE - Membranous, small 1/2 mm. or less, obtuse-toothed. COLLAR - Glabrous. SHEATH - Short canescent to glabrous, elliptical, the margin hyaline. NODE Glabrous. INTERNODE - Glabrous. ROOTS - Fibrous. CULM - Elliptical. OUTSTANDING CHARACTERS - Blade long, narrow and folded. Ligule small, obtuse-toothed.

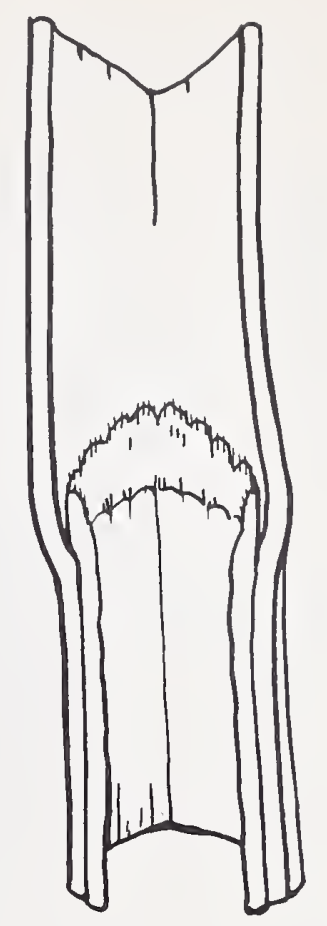

60. Poa longiligula Scribn. \& Will. LONGTONGUE MUTTON BLUEGRASS

Erect, small bunchgrass. VERNATION - Folded. BLADES - Folded, erect, narrow, pointed; smooth; ribs prominent dorsally; veins indistinct; midrib prominent ventrally; width $1 \mathrm{~mm}$., length $10-20 \mathrm{~cm}$., the tip boat-shaped. AURICLE - None. LIGULE - Membranous, 2-7 mm., acute-lacerate. COLLAR - Glabrous. SHEATH - Glabrous, elliptical. NODE - Glabrous. INTERNODE - Glabrous. ROOTS - Fibrous. CULM Elliptical, not branched. OUTSTANDING CHARACTERS - Blade long, narrow and folded. Ligule prominent, 2-4 nm., acutelacerate. Blade ribs prominent dorsally.
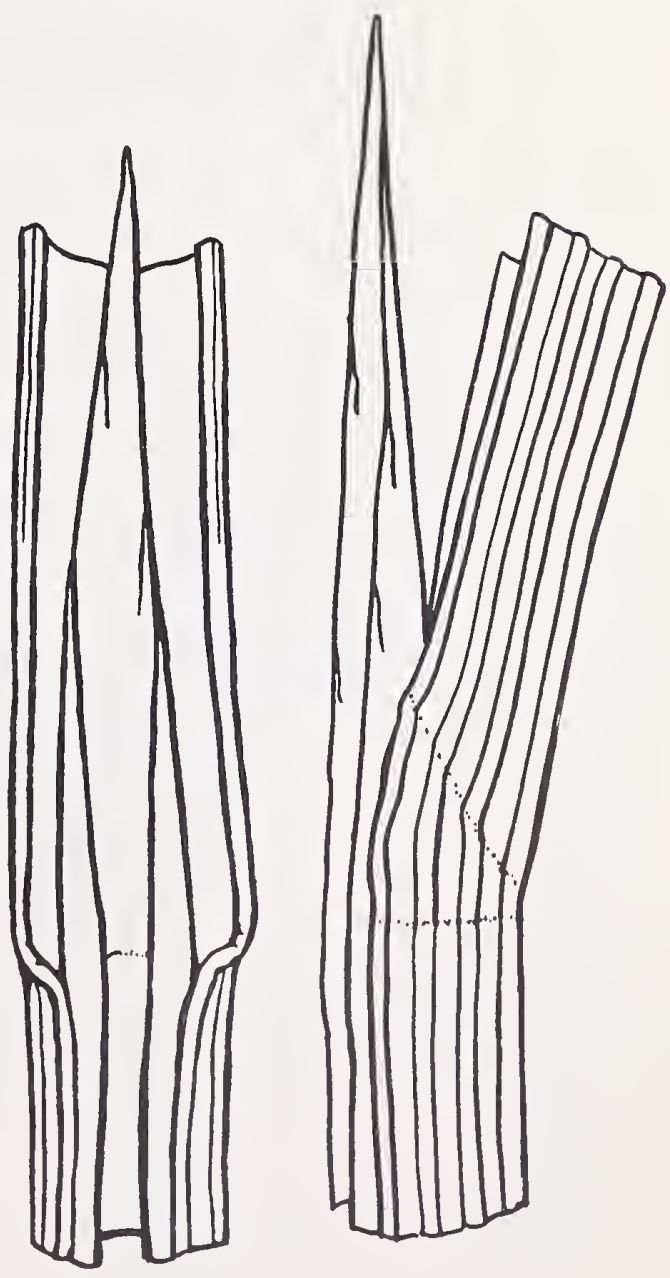
Erect, sodgrass. VERNATION - Folded. BLADES - Folded, soft; ribs prominent ventrally; margin smooth; width $1 \mathrm{~mm}$., length 10-20 cm., the tip boat-shaped. AURICLE - None. LIGULE - Membranous, collar-like, small 1/4-1/2 mm., truncateentire. COLLAR - Glabrous, not prominent. SHEATH - Glabrous, veined, elliptical, with membranous edges. NODE Glabrous. INTERNODE - Glabrous. ROOTS - Short rhizomes. CULM - Flat, not branched. OUTSTANDING CHARACTERS Blades long, ribbed, folded. Ligule very small, truncate-entire. Short rhizomes.

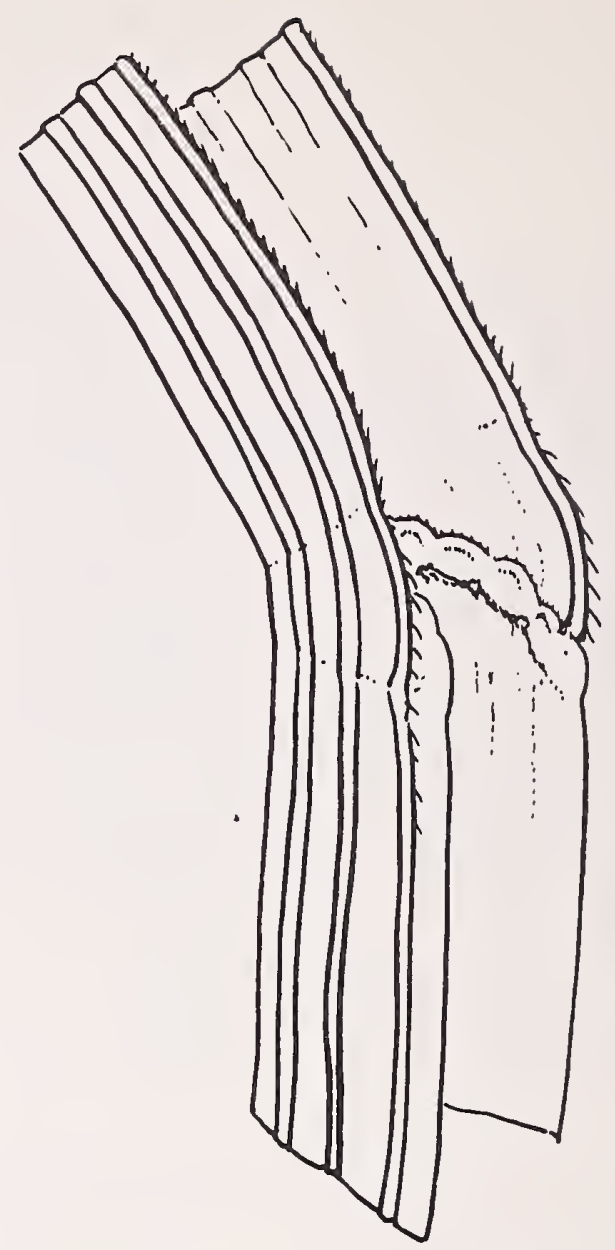

62. Schedonnardus paniculatus (Nutt.) Trel. TUMBLEGRASS

Erect, small bunchgrass. VERNATION - Folded. BLADES - Folded, twisted, erect, narrow, pointed; smooth, soft; veins each side of midvein 1-2; ribs indistinct; margin white, toothed; midrib prominent dorsally; width $1 \mathrm{~mm}$., length $2-3 \mathrm{~cm}$. COLLAR - Smooth. AURICLE - None. LIGULE - Membranous, 2-3 mm., acute-entire. SHEATH - Flat, smooth, veined, paperlike white margined. NODE - Glabrous. INTERNODE - Glabrous. ROOTS - Fibrous. CULM - Flat, not branched. OUTSTANDING CHARACTERS - Blade and sheath conspicuous white margin. Midrib prominent ventrally. Blade short, twisted. Ligule prominent extending down sheath margin.

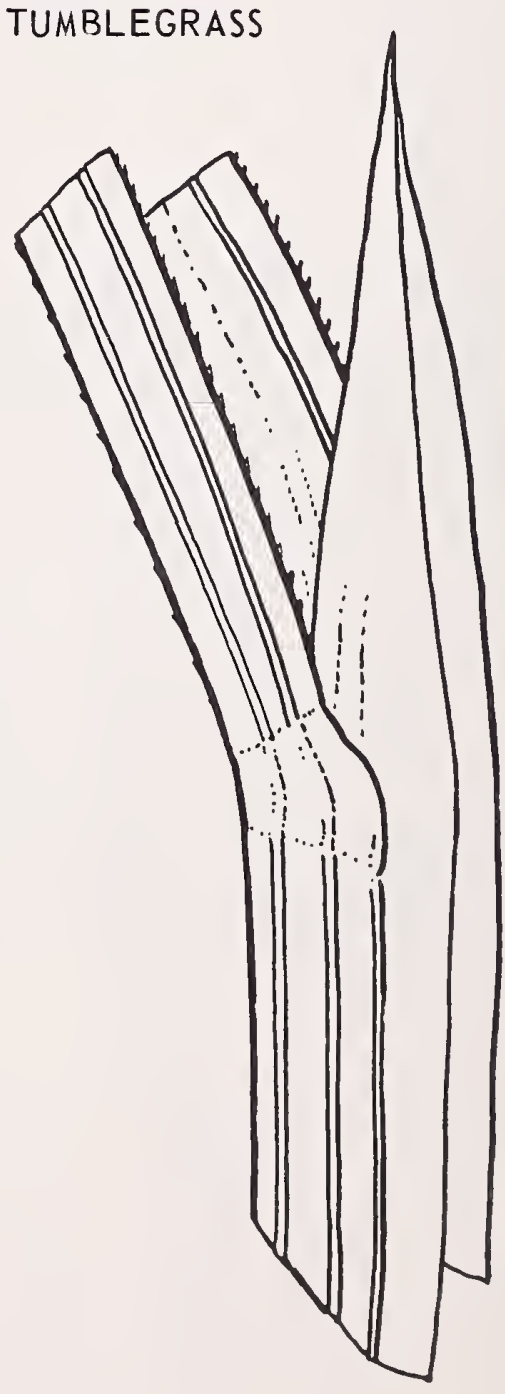


Semidecumbent, small annual bunchgrass. VERNATION - Curled. BLADES Rolled, narrow, short; hairy ventrally 3-8 mm.; too small to determine veins and ribs; width 1/2-1 mm., length $3-8 \mathrm{~cm}$. AURICLE - None. LIGULE - Hairy, small 1-2 $\mathrm{mm}$. with scattered hairs $3-5 \mathrm{~mm}$. COLLAR - Hairs 1-5 mm. SHEATH Glabrous, veined, hyaline margin. NODE Glabrous. INTERNODE - Glabrous. ROOTS - Fibrous, annual. CULM - Round, not branched. OUTSTANDING CHARACTERS Annual semidecumbent growth. Long hairs on ligule and collar. Blades rolled, very narrow and short. Roots annual.

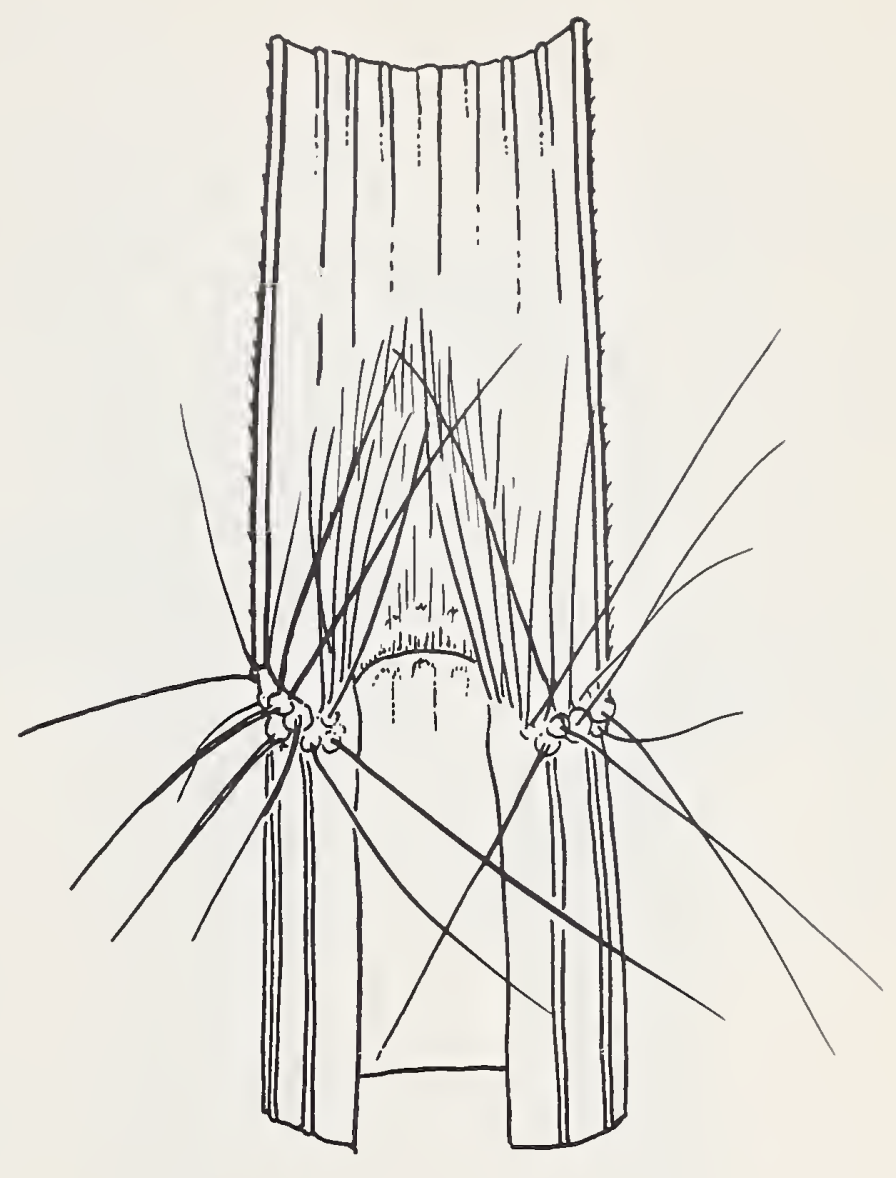

64. Setaria geniculata (Lam.) Beaur. KNOTROOT BRISTLEGRASS

Erect, large bunchgrass. VERNATION - Curled. BLADES - Folded, wide, long, drooping; hairy ventrally near ligule, these occasionally glandular; veins each side of midrib 4; ribs prominent; margin toothed; midrib not prominent; width 3-5 $\mathrm{mm}$., length 10-20 cm. AURICLE - None. LIGULE - Hairy, 1-2 mm., bearded. COLLAR - Hairy. SHEATH - Margin and lower nodes, culm hairy. NODE - Hairy. INTERNODE - Hairy. ROOTS - Fibrous. CULM Elliptical, branched. OUTSTANDING CHARACTERS - Ligule, node and lower internode hairy. Sheath margin hairy. Collar hairy. Blades folded.

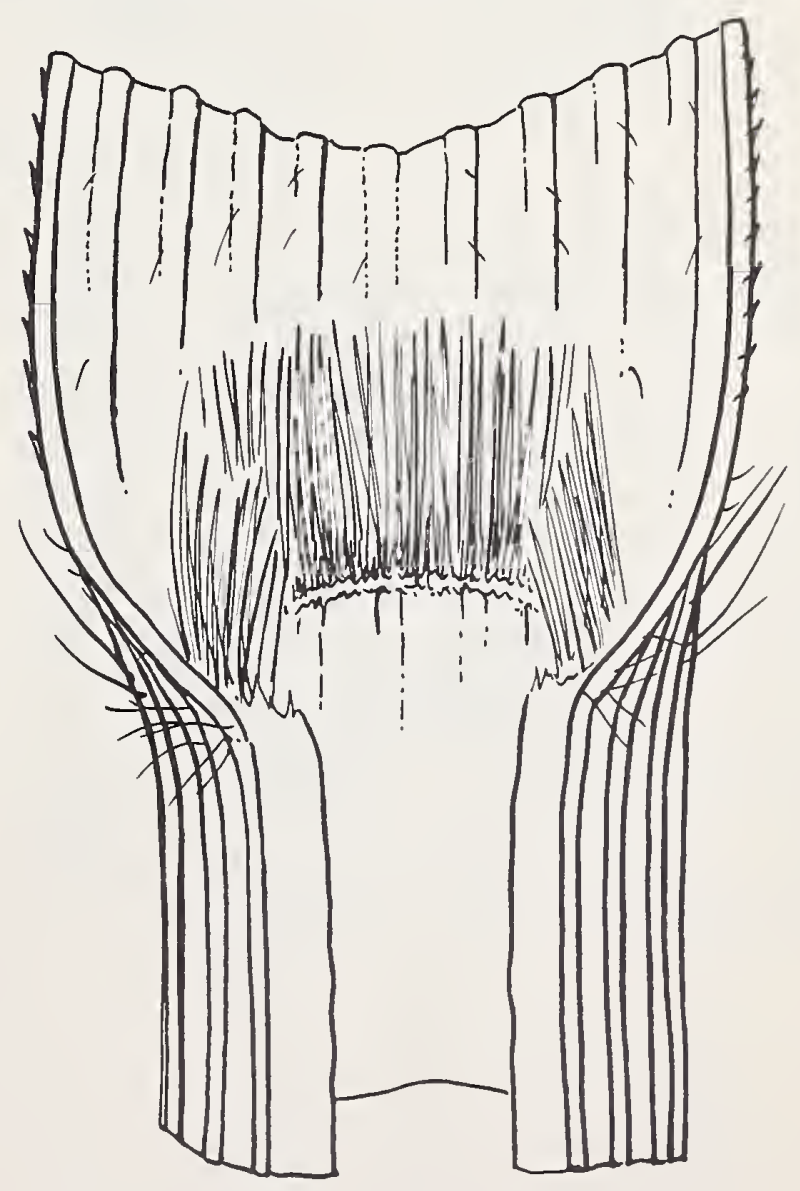


Erect, lower semi-erect, small bunchgrass. VERNATION - Curled. BLADES Flat, drooping, narrow, long; rough, soft, thin, veins each side of midrib $3-4$; ribs prominent with lens; margin light color; midrib prominent ventrally; width 2-3 mm., length 5-20 cm. AURICLE - None. LIGULE - hairy, small 1/2-1 mm. COLLAR Hairy margin, divided. SHEATH - Occasionally hairy margin. NODE - Glabrous to hairy. INTERNODE - Glabrous to hairy near nodes. ROOTS - Fibrous. CULM Flat, not branched. OUTSTANDING CHARACTERS - Culms flat. Blade in bud curled. Blade flat, long drooping. Ligule hairy, medium 1-2 $\mathrm{mm}$. Sheath occasional marginal hairs.

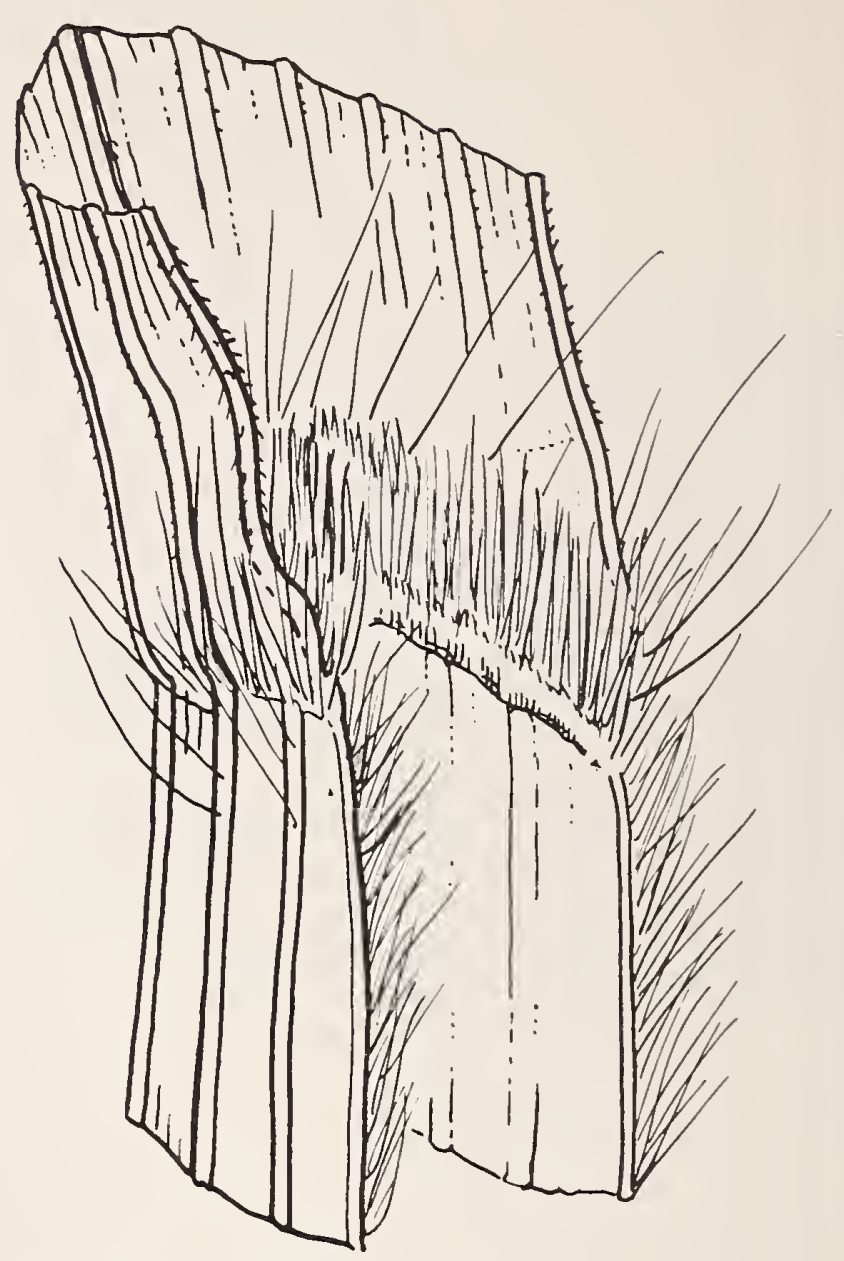

66. Sitanion hystrix (Nutt.) J. G. Smith. BOTTLEBRUSH SQUIRRELTAIL
Semi-erect, large bunchgrass. VERNATION - Curled. BLADES - Usually flat, twisted, drooping, narrow, pointed; rough, pubescent to glabrous; veins each side of midrib, 2-4; ribs prominent dorsally; 2-3 $\mathrm{mm}$. wide, 5-20 cm. long. AURICLE - Small $1 \mathrm{~mm}$. LIGULE - Membranous, small $1 / 4 \mathrm{~mm}$., truncate-entire. COLLAR - Glabrous, divided. SHEATH Pubescent or glabrous, ribbed, round frequently pinkish. NODES - Glabrous. INTERNODES - Glabrous. ROOTS - Fibrous. CULMi - Round, not branched. OUTSTANDING CHARACTERS - Auricle. Semidecumbent. Ribs prominent dorsally.

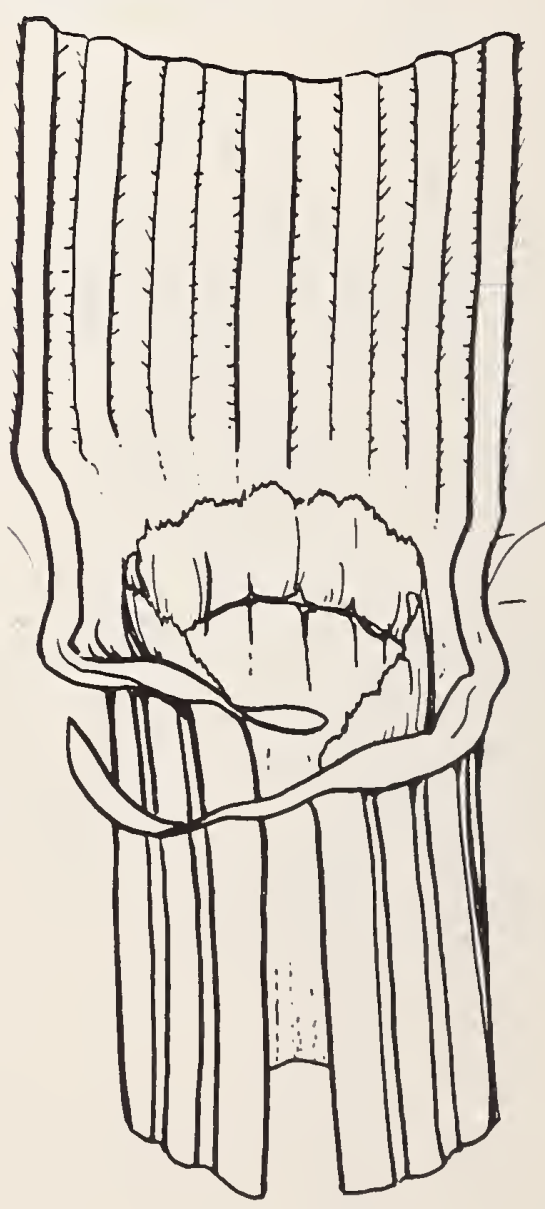


Erect, sod-like. VERNATION - Curled. BLADES - Flat, wide, drooping, narrow, pointed; veins each side of midrib 5-7; ribs indistinct; margin toothed, white; midrib prominent dorsally; width 10-15 mm., length 20-40 cm. AURICLE - None. LIGULE - Hairy, obtuse, 1-2 mm. COLLAR - Pubescent to glabrous. SHEATH Elliptical, veined. NODE - Glabrous to very short canescent. INTERNODE - Glabrous. ROOTS - Rootstalk rhizomatous. CULM - Elliptical, occasionally branched. OUTSTANDING CHARACTERS - Massive rootstalks rhizomes. Blade margin white. Blades large, flat. Prefers wet areas, roadside, irrigation ditches.

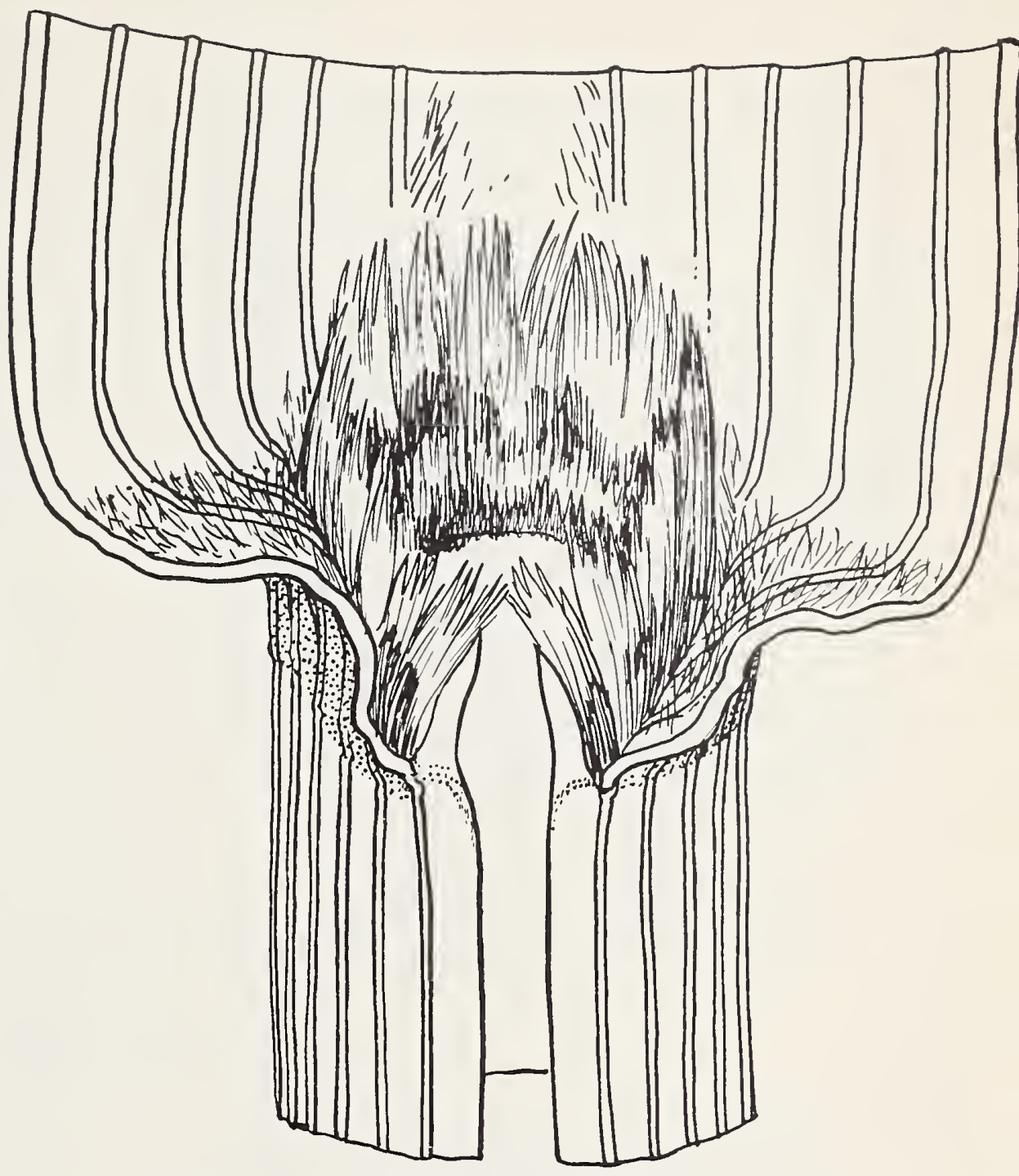

68. Sporobolus airoides Torr. ALKALI SACATON

Erect, large bunchgrass. VERNATION - Curled. BLADES - Rolled, drooping, narrow, pointed; glabrous, veins each side of midrib 3-4; ribs indistinct; margin glabrous; midrib not prominent; width $1 \mathrm{~mm}$., length 10-25 cm. AURICLE - None. LIGULE - Hairy, 1/2-1/4 mm., hairs on back of ligule 1-3 mm. COLLAR - Pubescent margin, divided. SHEATH - Round, glabrous, papery margin. NODE - Glabrous. INTERNODE - Glabrous. ROOTS - Short rhizomes. CULM - Round, not branched. OUTSTANDING CHARACTERS - Blade narrow, long, rolled. Ligule hairy, small 1/4-1/2 mm., long hairs at back 1-3 mm. Large bunches 1-2 feet when mature.

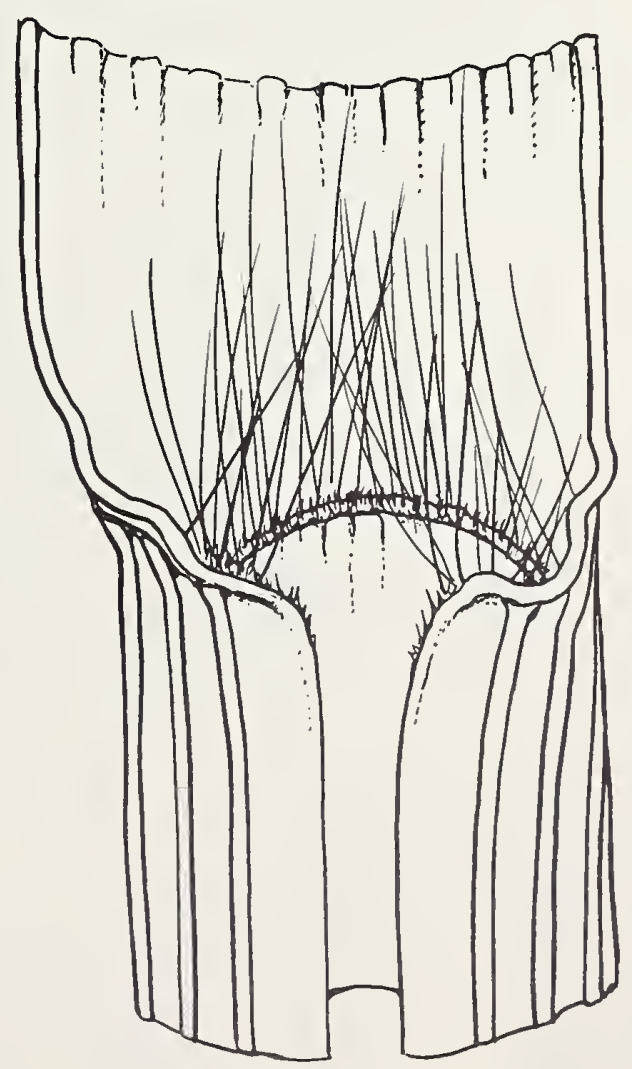


Erect, medium bunchgrass. VERNATION - Curled (sometimes reported as clasping). BLADES - Usually flat, narrow, pointed; glabrous, rough ventrally, soft; veins each side of midrib 2-3; margin toothed; ribs prominent ventrally and dorsally; width 2-3 mm., length 5-20 cm. AURICLE - None. LIGULE - Hairy, short. COLLAR - Tufted. SHEATH - Hairy margin 2-3 mm., usually pinkish at base. NODE - Glabrous. INTERNODE - Glabrous. ROOTS - Fibrous. CULM - Round, frequently branched at node. OUTSTANDING CHARACTERS - Blade flat, glabrous. Collar tufted. Sheath occasionally with marginal hairs. Panicles included well in sheath. Culm usually branched. In Arizona known only from Apache County.

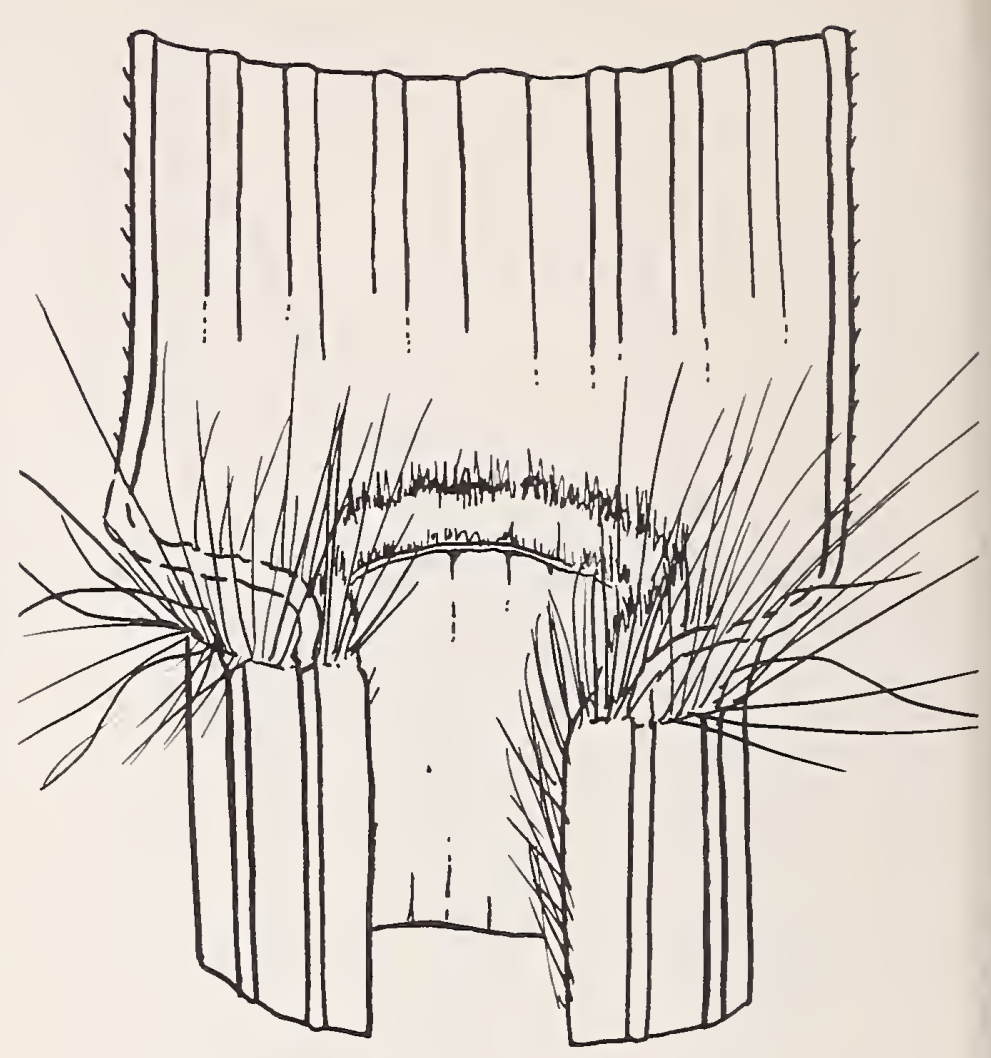

70. Sporobolus cryptandrus (Torr.) A. Gray. SAND DROPSEED
Erect, medium bunchgrass. VERNATION - Curled. BLADES - Flat, wide, long; glabrous; veins each side of midrib $3-4$; ribs prominent; margin toothed; midrib not prominent; width 3-8 mm., length $15-$ $20 \mathrm{~cm}$. AURICLE - None. LIGULE - Hairy, hairs 2-3 mm. on margin. COLLAR - Conspicuous tufts of hair on margin, $2-3 \mathrm{~mm}$. SHEATH - Hairy often on one margin, the panicle more or less covered by the sheath. NODE - Glabrous. INTERNODE Glabrous. ROOTS - Fibrous. CULM Round, not branched. OUTSTANDING CHARACTERS - Blades flat, long. Panicle included in sheath. Ligule and collar bearded. Culms not branched. Almost throughout the state, 150-7,000 feet elevation.

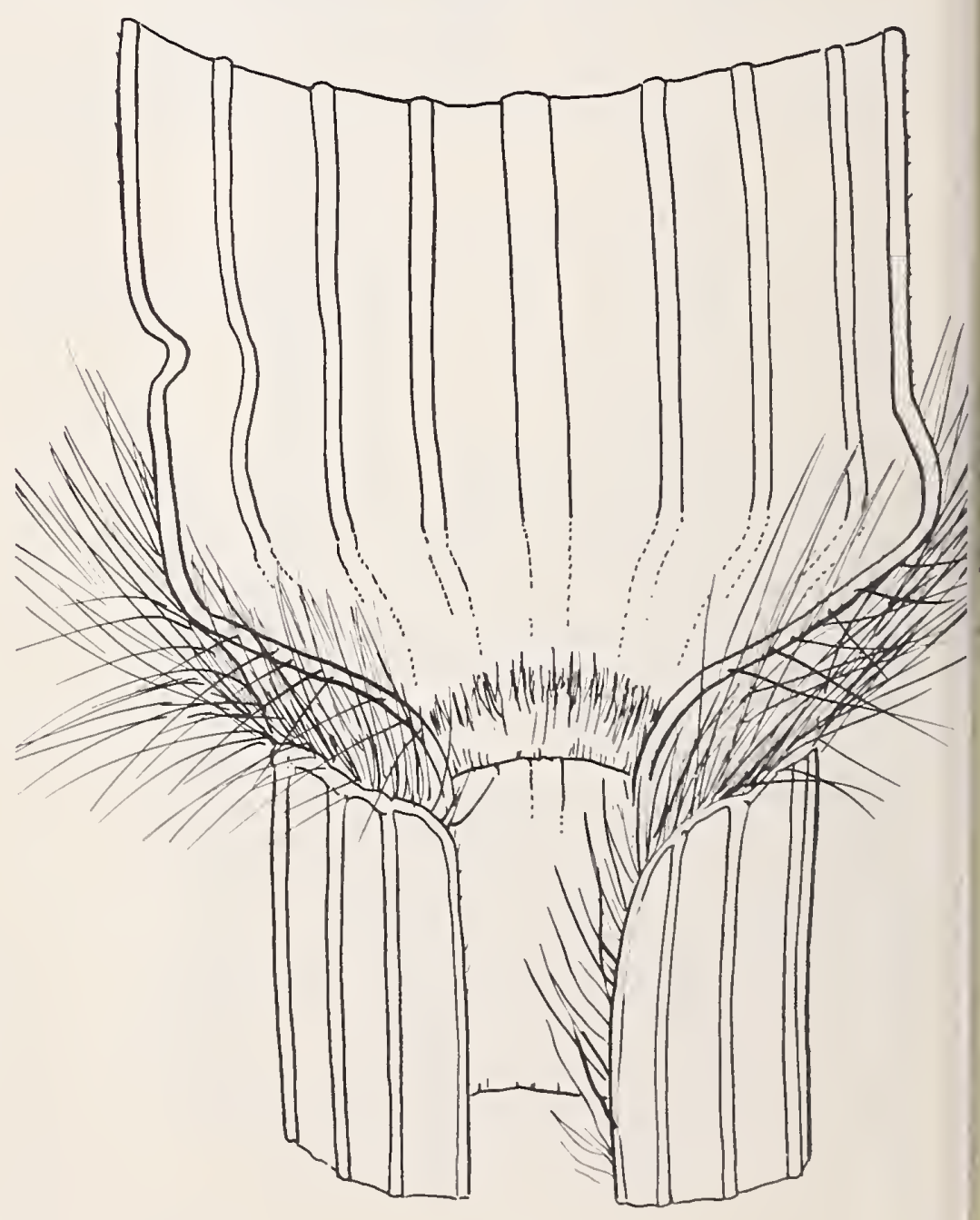


Erect, medium bunchgrass. VERNATION - Folded (reported as curled in Colorado). BLADES - Rolled, drooping, narrow, pointed; glabrous; veins indistinct; midrib not prominent; width $1 / 2-1$ mm., length 25-35 cm. AURICLE - None. LIGULE - Membranous, very small $1 / 4$ mm., truncate-ciliate. COLLAR - Glabrous, margin appearing hairy, divided, indistinct. SHEATH - Elliptical, veined. NODE - Glabrous. INTERNODE - Glabrous. ROOTS - Fibrous. CULM - Elliptical, not branched. OUTSTANDING CHARACTERS-Blades long, needle-like. Ligule very small, $1 / 4 \mathrm{~mm}$., truncate-ciliate. Awns long, twisted with long hairs toward tip.

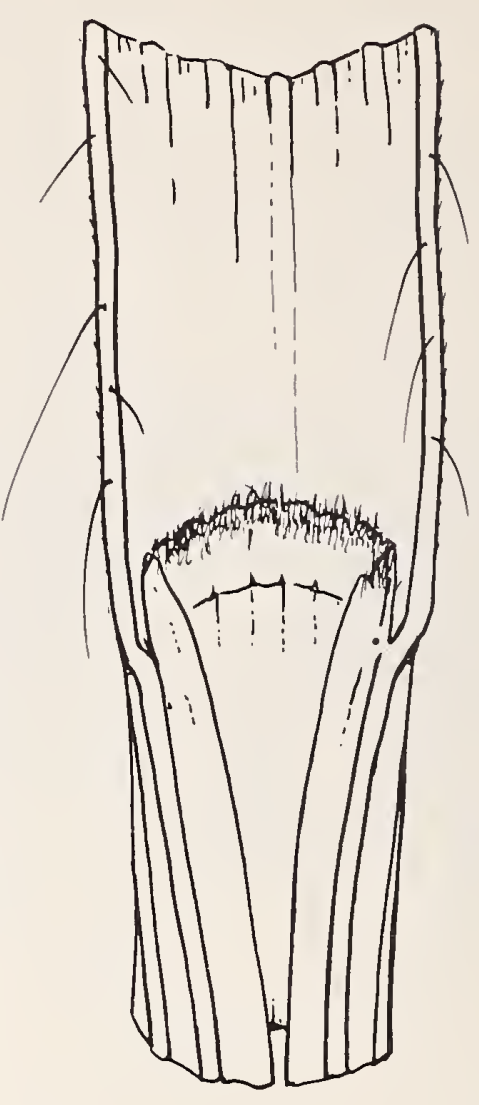

74. Stipa pringlei Scribn. PRINGLE NEEDLEGRASS

Erect, small bunchgrass. VERNATION - Curled. BLADES - Flat, drooping, narrow, pointed; rough ventrally; veins each side of midrib 2; ribs prominent ventrally and dorsally; margin toothed; midrib prominent ventrally; width $2 \mathrm{~mm}$., length 20-30 cm. AURICLE - None. LIGULE Membranous, small 1-2 mm., obtuselacerate. COLLAR - Glabrous. SHEATH Elliptical, veined, margin hyaline. NODE Usually pubescent. INTERNODE - Glabrous to short canescent near nodes. ROOTS - Fibrous. CULM - Elliptical, not branched. OUTSTANDING CHARACTERS Blades flat, narrow, long. Ligule membranous, small 1-2 mm., obtuse-lacerate. Awns twisted $1-3 \mathrm{~cm}$.
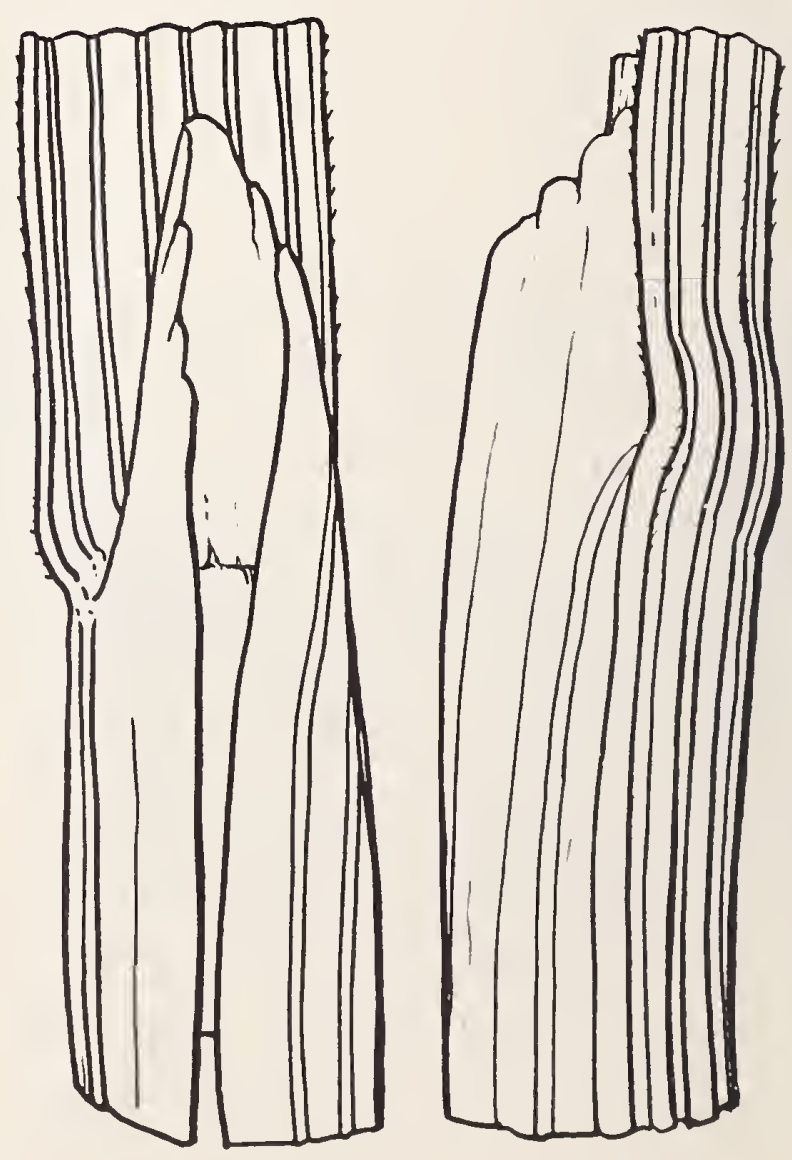
Erect, large bunchgrass. VERNATION - Curled. BLADES - Flat, drooping, narrow, pointed; glabrous or pubescent ventrally and dorsally, occasional glandular hairs dorsally; veins each side of midrib 3; ribs prominent ventrally; margin toothed, white; midrib prominent; width $3-4 \mathrm{~mm}$., length 8-12 cm. AURICLE - None. LIGULE - Membranous, 2-3 mm., obtuse-lacerate. COLLAR - Hairy ventrally, entire. SHEATH - Glabrous or pubescent, elliptical, margin hairy. NODE - Pubescent, occasionally glabrate. INTERNODE - Glabrous. ROOTS - Fibrous. CULM - Elliptical, branched. OUTSTANDING CHARACTERS - Blade margin white. Blades flat, long. Culms branched. Ligule 2-3 mm., obtuse-lacerate.

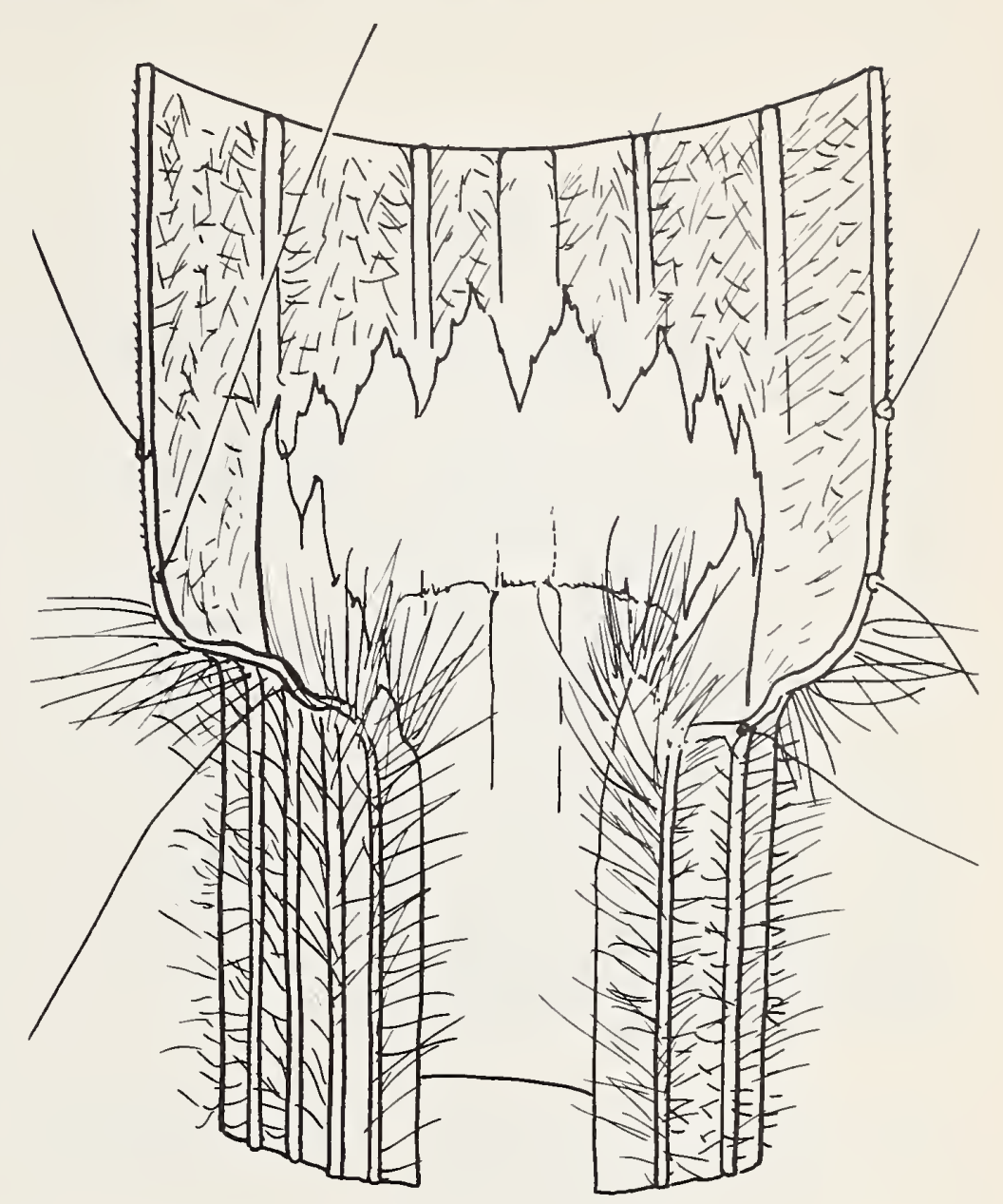

76. Tridens muticus (Torr.) Nash. SLIM TRIDENS
Erect, small bunchgrass. VERNATION - Curled. BLADES - Rolled, erect, narrow, pointed; with occasional glandular hairs dorsally; scabrous; midrib prominent dorsally; veins each side midvein 4-5; width 2-4 mm., length 8-20 cm. AURICLE None. LIGULE - Hairy, small 1/2-1 mm. COLLAR - Hairy. SHEATH - Open, hairy on margin, round. NODE - Pubescent. INTERNODE - Glabrous. ROOTS - Fibrous. CULM - Round. OUTSTANDING CHARACTERS - Blade usually rolled. Ligule hairy and small. Node pubescent.

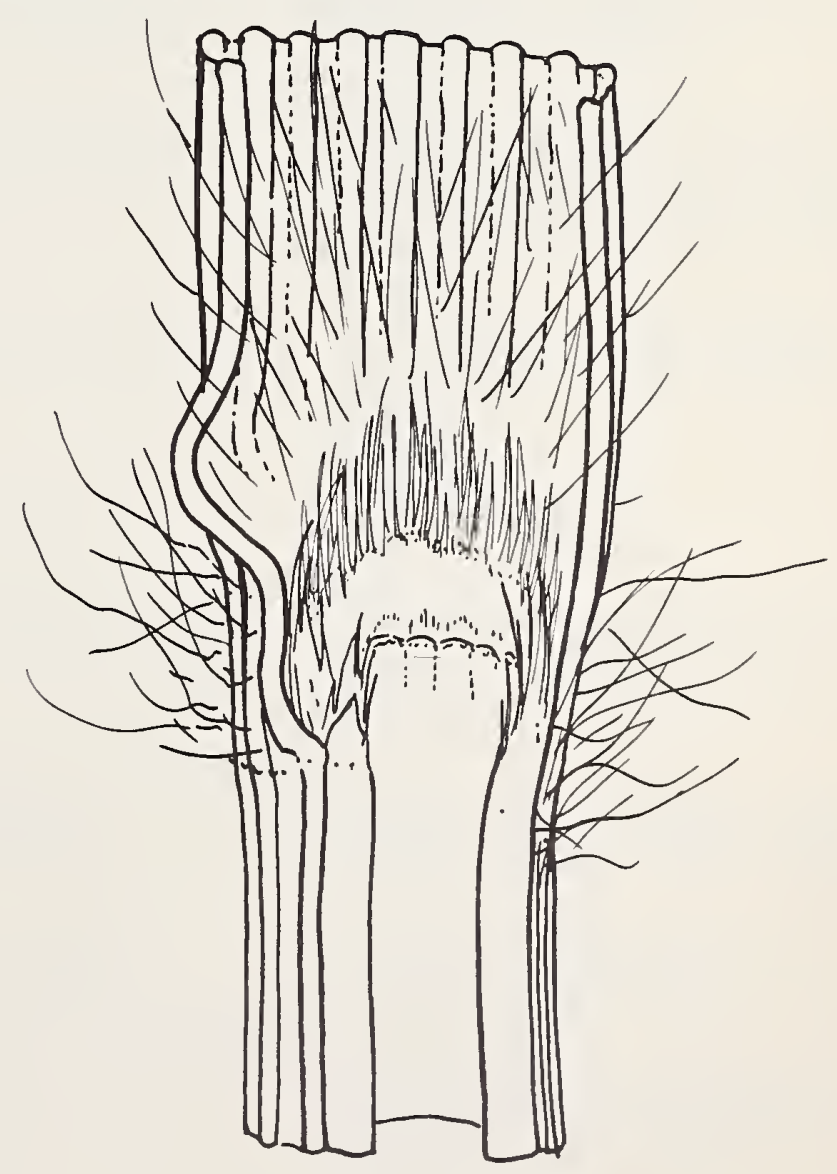


Erect, small bunchgrass. VERNATION - Folded. BLADES - Rolled, erect, very small, narrow, pointed; rough ventrally; ribs distinct, veins indistinct; margin toothed; width $1 / 4 \mathrm{~mm}$., length $2-5 \mathrm{~cm}$. AURICLE - None. LIGULE - Hairy, small 1/2 mm. COLLAR - Smooth, small, entire. SHEATH - Margin paper-like, the lower long ciliate. NODE - Glabrous, roots at nodes. INTERNODE - Glabrous to pubescent. ROOTS - Fibrous. CULM - Elliptical, terminal node branched with cluster of flowers often cobwebby. OUTSTANDING CHARACTERS - Blades ribbed, rolled, small and short. Sheath margin conspicuously paper-like, long ciliate. Flowers clustered, somewhat exceeded by the leaves, cobwebby. Roots at nodes.

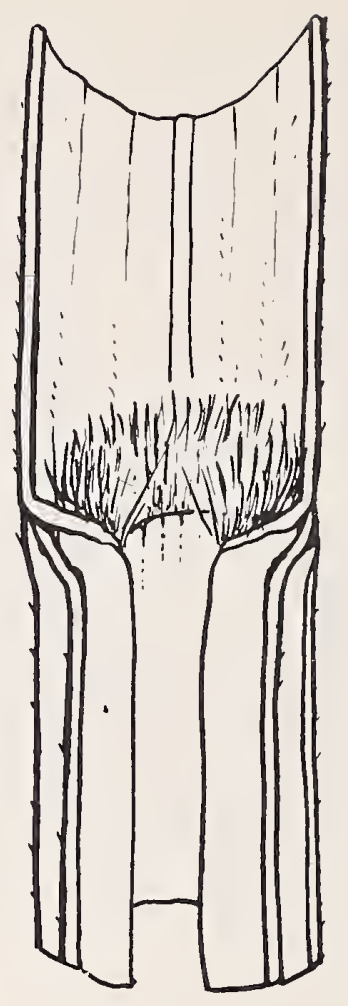


ACUTE: Terminating sharply in an angle of less than $90^{\circ}$.

AURICLE: Small, membranous, clawlike extension of the collar of some grasses.

AWN:

A needle-like extension of the grass floret, as in Stipa (needlegrass) or Aristida (three-awn).

CANESCENT: Grayish or whitish due to dense, fine hairs.

CILIATE:

COLLAR:

CORM:

CULM:

DECUMBENT: Reclining on the ground

DIVIDED: but with the end ascending, bending horizontally at the base. Said of stems. Decumbent conveys the idea of weakness.

Fringed with hairs on the edge.

The region on the outer side of the grass leaf at the junction of the sheath and blade.

A swollen or enlarged, rounded, solid, fleshy, mostly subterranean, stem base. Like a bulb in shape and appearance, except that it is solid instead of being composed of fleshy scales.

The jointed stalk or stem of grasses, usually hollow except at the nodes, and mostly herbaceous.

Refers to the shape of the collar on the back of the sheath.
DORSAL:

ELLIPTICAL:

ENTIRE:

FIBROUS:

FLORET:

GENICULATE:

GLABRATE:

GLABROUS:

GLOSSY:

HIRSUTE:

HISPID:

HYALINE:

INFLORESCENCE: The flowering portion of a

Refers to the top of the blade, opposite to ventral.

The cross section of the stem partially flattened.

With an even margin, without teeth or lobes.

Refers to a mass of threadlike roots.

A unit of the spikelet of a grass inflorescence, consisting usually of lemma, palea, stamens, and pistil.

Knee-like; bent like a knee; said of stems and awns of needlegrass (Stipa).

Becoming glabrous or nearly so in age.

Devoid of hairs or pubescence; smooth in the sense of absence of all hairiness.

GLANDULAR: Pertaining to or possessing glands at the base of hairs.

Smooth, shining.

Hairy with rather coarse, stiffish, straight, beardlike hairs.

Bristly; beset with stiff, rough, bristle-like hairs.

Thin and translucent. grass plant, consisting of spikelets and florets.


INTERNODE:

INVOLUTE:

LACERATE:

LANCEOLATE:

LIGULE:

MAT:

MIDRIB:

NODE:

NOTCHED:

OBTUSE:
The portions of a stem between the nodes, or joints.

Inrolled; i.e. with both edges rolled in toward the middle (blade) each edge presenting a spiral appearance in cross-section.

PERENNIAL:

PILOSE: times longer than broad, and tapering from the relatively narrow base to apex.

The projecting, usually tongue-like, membranous end of the lining of the leaf sheath, seen at the base of the leaf, between it and the stalk, and a very characteristic feature of the grass family. The ligule is quite often an important means of distinguishing grasses; sometimes it is reduced to a mere fringe of hairs or to a hardened ring.

Growing thickly, or closely interwoven.

The central or main rib common in blades of grasses.

A joint or knot. Said especially of stems, whose nodes or joints are enlarged, often dark colored, and are the points whence leaves and additional flowers often spring.

An edge with teeth or lobes.

Blunt or rounded at the tip; not sharply pointed.

PANICLE:

PLUMED:

PUNGENT:

RACEME:

RACHIS:

RETRORSE:

RHIZOME:

RIB:

RUNNER:
An open inflorescence in which the lower branches are typically longer and blossom earlier than the upper branches.

Lasting for three or more years; said especially of herbaceous plants that are neither annual nor biennial.

Hairy with soft, slender hairs.

PUBESCENT: Clothed with soft hairs or down.

Sharply and rigidly pointed.

A simple, elongated, indeterminate flower cluster.

The axis of a spike, raceme, or branch of a panicle; the organ that supports the florets.

Directed back or downward, as hairs on the sheath of certain grasses.

An underground stem; recognized by the regular nodes and internodes; e.g. Sorghum halepense.

The primary veins of a blade.

\section{RUDIMENTARY: Reduced.}

A long, slender form of creeping branch, prostrate on the ground. Each runner, after having grown to its full length, strikes root from the tip (it sometimes roots at the joints also, in 
which case it may merge into a stolon), fixing the tip to the ground, then forms a bud at that point, which later develops into a tuft of leaves and so gives rise to a new plant.

SCABROUS: Rough or harsh to the touch.

Margin of a blade that buckles, resulting in waves.

SHEATH: In grasses, the lower part of the leaf which envelops the stem or culm.

SHREDDED: Deeply and irregularly cut along the edges.

SOD:

A group of plants growing densely together forming a solid mat, as buffalograss or Kentucky bluegrass grown for lawns.

SOLID: Applied to the culm, which is solid in a few grasses and all sedges and rushes.

SPIKELET: In grasses, the name applied to the cluster of one or more florets.
TOOTHED:

STOLON:

TOOTHED:

TRUNCATE:

VEIN:

VENTRAL:

VILLOUS:

WOOLLY:
A trailing or reclining branch, above ground, which takes root where it touches the soil, there sending up new shoots which later become separate plants.

An edge with regular notches.

Squared at the tip; terminating abruptly as if cut off crosswise.

One of the fibrovascular bundles forming part of the framework (skeleton) of a leaf; more or less parallel veinations (as in a grass blade).

Refers to the inner surface of the blade, facing the culn as the leaf is held erect.

Densely hairy. with long, soft hairs.

Covered with long, matted hairs. 
. 


\section{REFERENCE PUBLICATIONS}

Copple, R. F., and Aldous, A. E.

1932. The identification of certain native and naturalized grasses by their vegetative characters. Kans. Agr. Exp. Sta. Tech. Bull. 32, 73 pp., illus.

Contains keys, descriptions, and drawings of 26 species of Kansas grasses, many of which occur in Arizona.

Gould, F. W.

1951. Grasses of the southwestern United States. Univ. Ariz. Biol. Sci. Bull. 7, 352 pp., illus.

Harrington, H. D., and Durrell, L. W. 1944. Key to some Colorado grasses in vegetative condition. Colo. Agr. Exp. Sta. Tech. Bull. 33, 86 pp., illus. Contains keys, descriptions, and drawings of 119 species of grasses, many of which occur in Arizona.
Hitchcock, A. S.

1950. Manual of the grasses of the United States. U.S. Dep. Agr. Misc. Pub. 200, Ed. 2, revised by Agnes Chase. 1,050 pp., illus.

Humphrey, Robert R.

1958. Arizona range grasses. Ariz. Agr. Exp. Sta. Bull. 298, 104 pp., illus. Contains drawings and descriptions, with information on distribution, forage value, and management of the grasses included in the present publication.

Kearney, T. H., and Peebles, Robert $\mathrm{H}$.

1951. Arizona flora. 1,032 pp., illus. Berkeley \& Los Angeles: Univ. Calif. Press. 
Figure and Species

Description Number

Agropyron desertorum

Agropyron smithii

Agrostis alba

Andropogon barbinodis

Andropogon cirratus

Andropogon scoparius

Alkall sacaton

Aristida adscensionis

Aristida divaricata

Aristida glabrata

Aristida grouca

Aristida Zongiseta

Aristida purpurea

Aristida termipes

Ar1zona brome

Ar1zona cottontop

Arizona fescue

Bermudagrass

Blgelow bluegrass

Big galleta

Black grama

Blepharoneuron tricholepis

Blue grama

Blue three-awn

Bottlebrush squirreltall

Bouteloua aristidoides

Bouteloua barbata

Bouteloua chondrosioides
1

2

3

4

5

6

68

7

8

9

10

11

12

13

25

75

36

31

58

41

19

14

21

10

66

15

16

17
Figure and Specles

Description Number

Bouteloua curtipendula

18

Boutelona exiopoda

19

Bouteloua filiformis

20

Boutelona gracilis

21

Bouteloua hirsuta

22

Bouteloua rothrockii

23

Bromus anomalus $\quad 24$

Bromus arizonicus 25

Bromus ciliatus 26

Bromus inermis 27

Bromus rubens 28

Bromus tectorum 29

Bulb panfcum 55

Bullgrass $\quad 47$

Bush muhly $\quad 49$

Cane bluestem 4

Cheatgrass 29

Chzoris virgata $\quad 30$

Crested wheatgrass 1

Curlymesquite $\quad 38$

Cynodon dactylon $\quad 31$

Deergrass 52

Desert saltgrass 32

Distichlis stricta 32

Elyonurus barbiculmis 33

Eragrostis intermedia $\quad 34$

Eragrostis Zehonniana 35

Feather fingergrass $\quad 30$ 
Feather pappusgrass

57

Festuca arizonica

36

77

Fluffgrass

26

Fringed brome

39

Galleta

71

43

22

37

Heteropogon contortus

38

Hilaria belangeri

39

40

41

54

67

42

61

64

42

35

Lehmann lovegrass

43

Leptochloa dubia

Little bluestem

Lolizm perenne

Longtongue mutton bluegrass

Lycums phleoides

Mat muhly

Medi terraneangrass

Mountain muhly

Muhlenbergia curtifolia

Muhlenbergia emersleyi

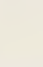

Muhlenbergia porteri

Muhlenbergia pungens 50

Muhlenbergia richardsonis 51

Muhlenbergia rigens $\quad 52$

Muhlenbergia torreyi 53

Mutton bluegrass 59

Needle grama $\quad 15$

New-Mexican feathergrass 73

Nodding brome $\quad 24$

Oryzopsis hymenoides $\quad 54$

Panicwn bulboswon 55

Panicum obtusum $\quad 56$

Pappophorw mucronulation 57

Perennial ryegrass 44

Pine dropseed $\quad 14$

Plains bristlegrass 65

Plains lovegrass 34

Poa bigelovii 58

Poa fendleriana 59

Poa Zongiligula 60

Poa pratensis $\quad 61$

Poverty three-awn 8

Pringle needlegrass $\quad 74$

Purple three-awn $\quad 12$

Red brome 28

Red three-awn 11

Red top 3

Ring muhly $\quad 53$

Rothrock grama 23

Sacaton $\quad 72$

$48 \quad$ Sand dropseed 70 
Sandhill muhly

Santa Rita three-awn

Schedonnardus paniculatus

Schismus barbatus

Setaria geniculata

Setaria macrostachya

Sideoats grama

Sitanion hystrix

Sixweeks grama

Sixweeks three-awn

Slender grama

SIIm trideris

Smooth brome

Sorghum halepense

Spidergrass

Sporobolus airoides

Sporobolus asper

sporobolus cryptandrus
50

9

62

63

64

65

18

66

16

7

20

76

27

67

13

68

69

70
Sporobolus giganteus

71

Sporobolus wrightii

72

Sprucetop grama

17

Stipa neomexicana

73

Stipa pringlei

74

Tall dropseed

69

Tanglehead

37

Texas bluestem

5

Tobosa

40

Trichachne californica

75

Tridens muticus

76

Tridens pulchellus

77

Tumblegrass

62

Utah muhly

46

Vine-mesquite

56

Western wheatgrass

2

Wolftall

45

Woolspike 


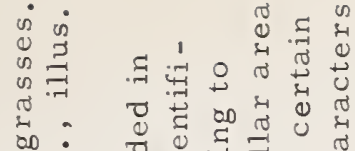

का : व

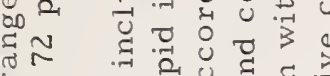

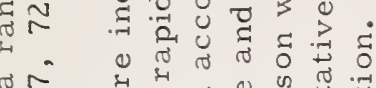

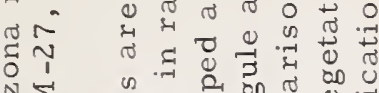

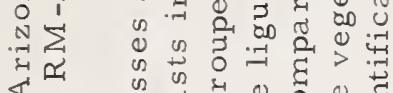

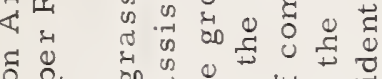

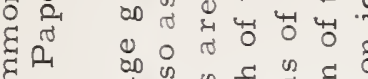

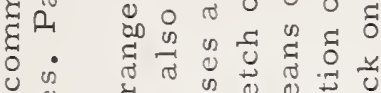

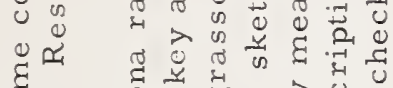

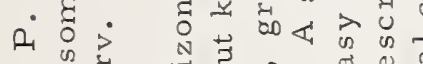

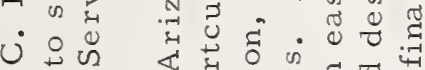

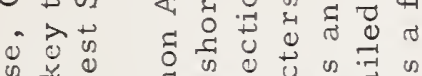

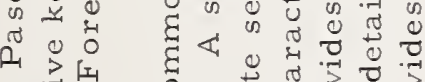

च

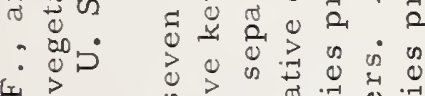

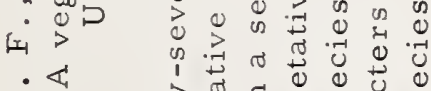

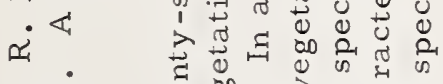

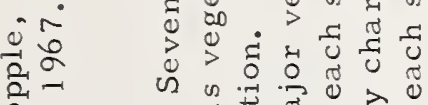

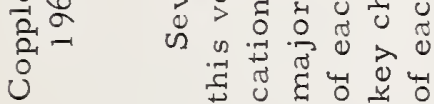

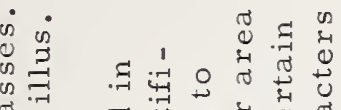

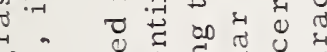

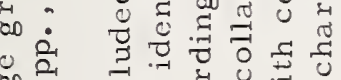

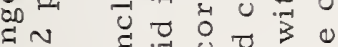

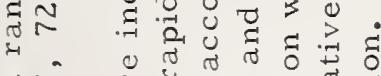

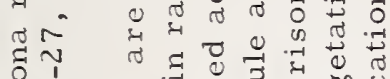

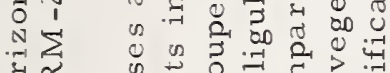

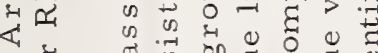

50 क्ष की

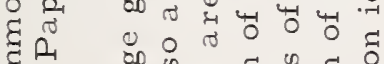

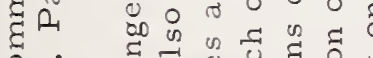

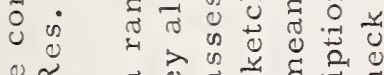

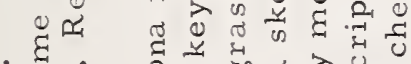

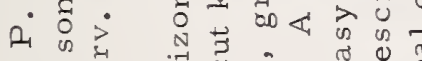

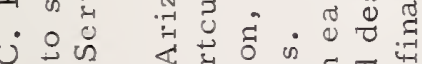

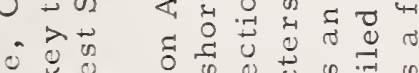

क

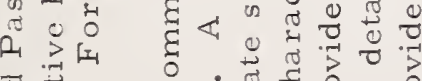

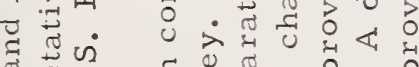

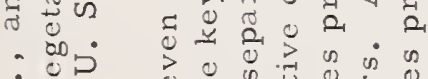

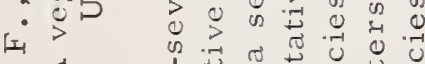

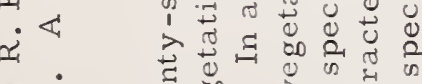

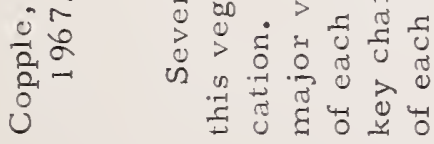

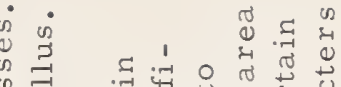

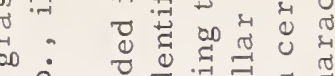

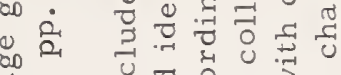

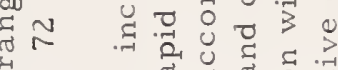

त

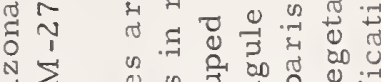

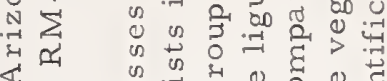

द्य 0 की

हो की

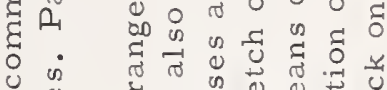

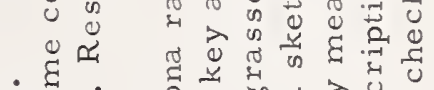

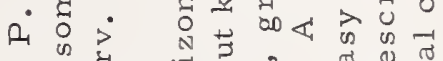

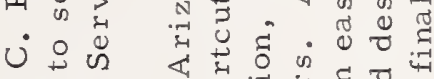

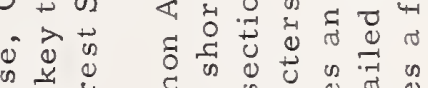

का दू की

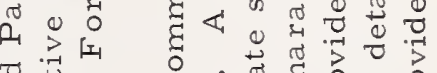

चี

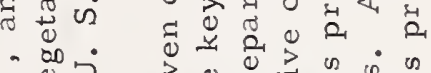

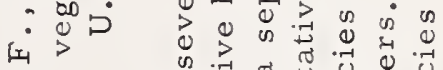

舟《

ĩ 0

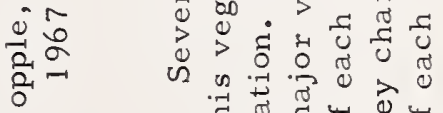

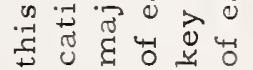

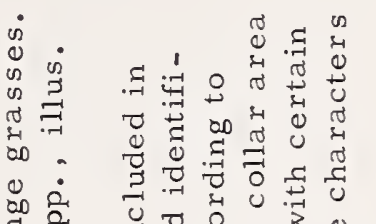

要

ॠ

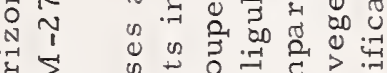

प्रिण की

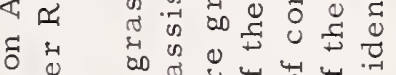

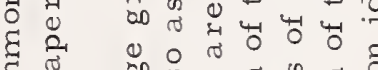

द्वृ का

0 प क्ष

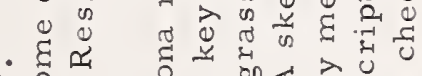

म.

ن엉 द्य

ज议

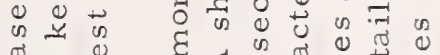

D.

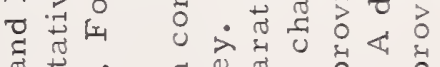

ฮ山

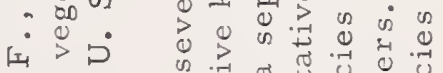

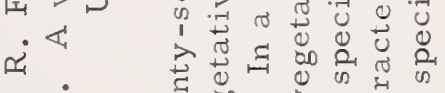

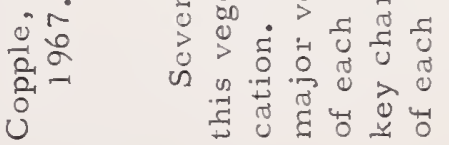





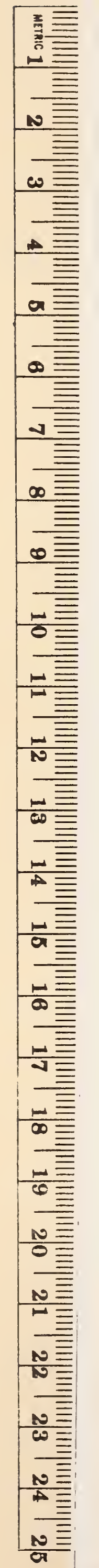




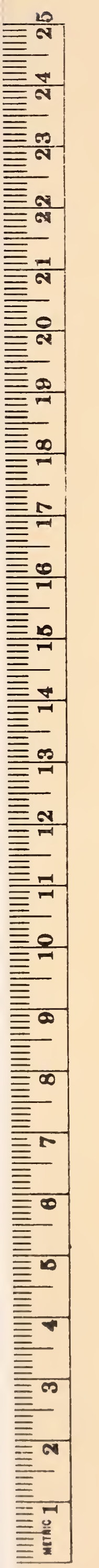

